\title{
Fluid Dynamics of Carbon Dioxide Disposal into Saline Aquifers
}

\author{
by \\ Julio Enrique García \\ Engineering (University of Los Andes, Bogotá, Colombia) 1991 \\ M.S. (University of Colorado at Boulder) 1995 \\ A dissertation submitted in partial satisfaction of the \\ requirements for the degree of \\ Doctor of Philosophy \\ in \\ Engineering - Civil and Environmental Engineering \\ in the \\ GRADUATE DIVISION \\ of the \\ UNIVERSITY OF CALIFORNIA, BERKELEY \\ Committee in charge: \\ Nicholas Sitar, Chair \\ Karsten Pruess \\ Tadeusz W. Patzek \\ Kent S. Udell
}

Fall 2003

This work was supported by the US Department of Energy through the Office of Basic Energy Sciences and through the National Energy Technology Laboratory (NETL) under Contract No. DE-AC03-76SF00098. 



\section{Fluid Dynamics of Carbon Dioxide Disposal into Saline Aquifers}

Copyright 2003

by

Julio Enrique García

The U.S. Department of Energy has the right to use this document for any purpose whatsoever including the right to reproduce all or any part thereof. 



\author{
Abstract \\ Fluid Dynamics of Carbon Dioxide \\ Disposal into Saline Aquifers \\ by \\ Julio Enrique García \\ University of California, Berkeley \\ Nicholas Sitar, Chair
}

Doctor of Philosophy in Engineering - Civil and Environmental Engineering

Injection of carbon dioxide $\left(\mathrm{CO}_{2}\right)$ into saline aquifers has been proposed as a means to reduce greenhouse gas emissions (geological carbon sequestration). Largescale injection of $\mathrm{CO}_{2}$ will induce a variety of coupled physical and chemical processes, including multiphase fluid flow, fluid pressurization and changes in effective stress, solute transport, and chemical reactions between fluids and formation minerals. This work addresses some of these issues with special emphasis given to the physics of fluid flow in brine formations.

An investigation of the thermophysical properties of pure carbon dioxide, water and aqueous solutions of $\mathrm{CO}_{2}$ and $\mathrm{NaCl}$ has been conducted. As a result, accurate representations and models for predicting the overall thermophysical behavior of the system $\mathrm{CO}_{2}-\mathrm{H}_{2} \mathrm{O}-\mathrm{NaCl}$ are proposed and incorporated into the numerical simulator TOUGH2/ECO2. The basic problem of $\mathrm{CO}_{2}$ injection into a radially symmetric brine aquifer is used to validate the results of TOUGH2/ECO2. The numerical simulator has been applied to more complex flow problem including the $\mathrm{CO}_{2}$ injection project at the Sleipner Vest Field in the Norwegian sector of the North Sea and the evaluation of fluid flow dynamics effects of $\mathrm{CO}_{2}$ injection into aquifers. 
Numerical simulation results show that the transport at Sleipner is dominated by buoyancy effects and that shale layers control vertical migration of $\mathrm{CO}_{2}$. These results are in good qualitative agreement with time lapse surveys performed at the site.

High-resolution numerical simulation experiments have been conducted to study the onset of instabilities (viscous fingering) during injection of $\mathrm{CO}_{2}$ into saline aquifers. The injection process can be classified as immiscible displacement of an aqueous phase by a less dense and less viscous gas phase. Under disposal conditions (supercritical $\mathrm{CO}_{2}$ ) the viscosity of carbon dioxide can be less than the viscosity of the aqueous phase by a factor of 15 . Because of the lower viscosity, the $\mathrm{CO}_{2}$ displacement front will have a tendency towards instability. Preliminary simulation results show good agreement between classical instability solutions and numerical predictions of finger growth and spacing obtained using different gas/liquid viscosity ratios, relative permeability and capillary pressure models. Further studies are recommended to validate these results over a broader range of conditions. 
To my parents, Gonzalo and Yolanda

To my brothers and sister, Gonzalo José, Gerardo and Ana Beatriz

To Laura Fogli, of course 



\section{Contents}

List of Figures $\quad$ V

List of Tables $\quad$ ix

1 Introduction $\quad 1$

1.1 Background and Motivation . . . . . . . . . . . . . 1

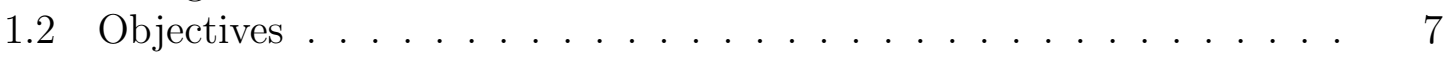

1.3 Overview . . . . . . . . . . . . . . . . . . 8

2 Thermophysical properties of fluid mixture components 11

2.1 Introduction . . . . . . . . . . . . . . . . . . . 11

2.2 Thermophysical properties of pure $\mathrm{CO}_{2} \ldots \ldots \ldots$. . . . . . . . 12

2.2.1 Volumetric properties . . . . . . . . . . . . 13

2.2.2 Enthalpy...................... . . . 21

2.2.3 Viscosity . . . . . . . . . . . . . . . . . . . . . 27

2.3 Thermophysical properties of pure $\mathrm{H}_{2} \mathrm{O} \ldots \ldots$. . . . . . . . . 30

2.4 The system carbon dioxide-water . . . . . . . . . . . . . . . 31

2.4.1 Phase behavior in the system carbon dioxide-water . . . . . . 31

2.4.2 Solubility models . . . . . . . . . . . . . . . . . . 34

2.5 Thermophysical Properties of Fluid Mixtures . . . . . . . . . . . . . . 42

2.5.1 Density of aqueous solutions of $\mathrm{CO}_{2} \ldots \ldots \ldots$. . . . . . . . 42

2.5.2 Enthalpy of fluid mixtures . . . . . . . . . . . . . . . . 43

2.5.3 Viscosity of fluid mixtures . . . . . . . . . . . . . . . . . 44

2.6 Phase equilibria in $\mathrm{CO}_{2}-\mathrm{H}_{2} \mathrm{O}-\mathrm{NaCl}$ systems . . . . . . . . . . . . . . . . . . 44

2.7 Recommendations and concluding remarks . . . . . . . . . . . 45

3 Mathematical model and implementation 49

3.1 Introduction . . . . . . . . . . . . . . . . . . 49

3.2 Governing equations . . . . . . . . . . . . . . 50

3.2.1 Continuum balance equations . . . . . . . . . . . . 51

3.2.2 Accumulation terms . . . . . . . . . . . . . . 51

3.2 .3 Flux terms . . . . . . . . . . . . . . . . . . . 52

3.2.4 Closure relations ................... . . . . 54

3.3 Numerical implementation . . . . . . . . . . . . . . 58 
3.3.1 Space and time discretization $\ldots \ldots \ldots \ldots \ldots$

3.3 .2 Solution method . . . . . . . . . . . . . . . . . 60

3.4 ECO2 module description $\ldots \ldots \ldots$. . . . . . . . . . . . . . 61

3.4 .1 Program structure . . . . . . . . . . . . . . . . . . . . 62

3.4 .2 Phase diagnostic . . . . . . . . . . . . . . . . . 63

3.4.3 Modeling of permeability reduction . . . . . . . . . 63

$\begin{array}{lll}4 & \text { Code verification and applications } & 67\end{array}$

4.1 Introduction . . . . . . . . . . . . . . . . . . 67

4.2 Radial flow from a $\mathrm{CO}_{2}$ injection well . . . . . . . . . . . 68

4.2.1 Definition of the problem and input data . . . . . . . . 69

$4.2 .2 \quad \mathrm{CO}_{2}$ injection into a radial aquifer (no salinity) $\ldots \ldots \ldots .70$

$4.2 .3 \quad \mathrm{CO}_{2}$ injection into a radial saline aquifer . . . . . . . 74

$4.3 \mathrm{CO}_{2}$ injection into a 2-D layered brine formation . . . . . 78

4.3.1 Definition of the problem and input data . . . . . . . . 79

$4.3 .2 \quad \mathrm{CO}_{2}$ injection results $\ldots \ldots \ldots \ldots . \ldots \ldots$

4.4 Enhanced $\mathrm{CO}_{2}$ dissolution $\ldots \ldots \ldots \ldots \ldots \ldots$

4.4.1 Definition of the problem and input data . . . . . . . 86

4.4 .2 Simulation Results . . . . . . . . . . . . . . . . 86

5 Fluid dynamic instabilities $\quad 91$

5.1 Introduction . . . . . . . . . . . . . . . . . . 91

5.2 Mechanisms of Viscous Fingering _ . . . . . . . . . . . . . . . 93

5.3 Problem Formulation . . . . . . . . . . . . . . . . . . . . . . 95

5.3 .1 Governing Equations . . . . . . . . . . . . . . . 95

5.3 .2 Flow system . . . . . . . . . . . . . . . . . . . . . . 96

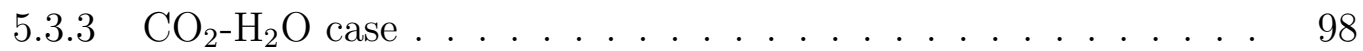

5.4 Numerical implementation . . . . . . . . . . . . . . . . . . . . . 104

5.4 .1 Methods of triggering fingering . . . . . . . . . . . . . 104

5.4 .2 Convergence under grid refinement . . . . . . . . . . . 106

5.4 .3 Boundary effects . . . . . . . . . . . . . . . . . . . . 110

5.4.4 Computer Performance . . . . . . . . . . . . . . . . 110

5.5 Results and applications . . . . . . . . . . . . . . . . . . . 112

$5.5 .1 \quad$ Finger spacing . . . . . . . . . . . . . . . . . . . . . . . 112

5.5 .2 Finger growth . . . . . . . . . . . . . . . . . . . 114

5.6 Viscous fingering simulations with ECO2 . . . . . . . . . 116

6 Conclusions 119

$\begin{array}{ll}\text { Nomenclature } & 127\end{array}$

$\begin{array}{ll}\text { Bibliography } & 139\end{array}$ 


\section{List of Figures}

2.1 Pressure-temperature diagram for pure carbon dioxide . . . . . . . . 14

2.2 Pressure-volume diagram for pure carbon dioxide . . . . . . . . . . 15

2.3 Calculated $\mathrm{CO}_{2}$ density $\left(\mathrm{kg} / \mathrm{m}^{3}\right)$ using the correlations of Altunin [1975] 20

2.4 Relative deviation in percentage for the density of $\mathrm{CO}_{2}$, (a) Vargaftik et al. [1996] vs. Altunin [1975] and (b) Span and Wagner [1996] vs. Altunin [1975] . . . . . . . . . . . . . . . . . . . . . . . . . . . . . 21

2.5 Calculated $\mathrm{CO}_{2}$ enthalpy $(\mathrm{J} / \mathrm{kg})$ using the correlations of Altunin [1975] 27

2.6 Relative deviation in percentage for the enthalpy of $\mathrm{CO}_{2}$ Vargaftik et al. [1996] vs. Altunin [1975] . . . . . . . . . . . . . . . . . . . . . 28

2.7 Calculated $\mathrm{CO}_{2}$ viscosity (Pa.s) using the correlations of Altunin [1975] 29

2.8 Relative deviation in percentage for the viscosity of $\mathrm{CO}_{2}$, (a) Vargaftik et al. [1996] vs. Altunin [1975] and (b) Fenghour et al. [1998] vs. Altunin [1975] . . . . . . . . . . . . . . . . . . . . . .

2.9 Pressure-temperature-composition diagram for the $\mathrm{CO}_{2}-\mathrm{H}_{2} \mathrm{O}$ system (from Tödheide and Franck [1963]). . . . . . . . . . . . . . . .

2.10 Enlarged P-T cross section of the $\mathrm{CO}_{2}-\mathrm{H}_{2} \mathrm{O}$ phase diagram, showing the two- and three-phase coexistence curves and the critical points, as presented by Spycher et al. [2003]. The circles are literature data points used by the authors in their mutual solubility model. The insert shows that the pure- $\mathrm{CO}_{2}$ liquid-vapor curve almost coincides with the threephase coexistence curve for the $\mathrm{CO}_{2}-\mathrm{H}_{2} \mathrm{O}$ system. $\mathrm{Q}_{1}$ is the quadruple point for the system. . . . . . . . . . . . . .

2.11 Pressure-composition cross section of the $\mathrm{CO}_{2}-\mathrm{H}_{2} \mathrm{O}$ phase diagram at $25{ }^{\circ} \mathrm{C}$. Circles represent data points for $\mathrm{CO}_{2}$ solubility in $\mathrm{H}_{2} \mathrm{O}$; squares are the for $\mathrm{H}_{2} \mathrm{O}$ solubility in $\mathrm{CO}_{2}$. . . . . . . . . . . . .

2.12 Temperature-composition diagram for the $\mathrm{CO}_{2}-\mathrm{H}_{2} \mathrm{O}$ system at 200 and 350 bars (from Bowers [1982]). Circles represent solubility data of Takenouchi and Kennedy [1964]; hexagons represent solubility data of Tödheide and Franck [1963]. . . . . . . . . . . . . . .

2.13 Fugacity coefficients for a range of temperature and pressure conditions. Lines are calculated from the correlation given by Spycher et al. [2003]; symbols represent the data of Angus et al. [1976] . . . . . . . 38

2.14 Temperature dependence of Henry's coefficient. . . . . . . . . . . . . 40 
2.15 Solubility of carbon dioxide in pure water at 20,31, 40, and $50{ }^{\circ} C$. Lines are calculated from Equation 2.52; symbols represent experimental data compiled by Spycher et al. [2003]. . . . . . . . . . . . 41

3.1 Space discretization in the integral finite difference method . . . . . . 59

3.2 Modular Architecture MULKOM of TOUGH2-ECO2 (FromPruess et al. [1999]) . . . . . . . . . . . . . . . . . . 62

3.3 TOUGH2-ECO2 structure based on callee and caller subroutines of EOS (From Fujitsu Visual Analyzer ${ }^{\complement}$ ). . . . . . . . . . . . . . . . . 65

4.1 Schematic of radial flow from a $\mathrm{CO}_{2}$ injection well . . . . . . . . 70

4.2 Simulated gas saturation front (no salinity). . . . . . . . . . . . 72

4.3 Simulated pressure (no salinity) . . . . . . . . . . . . . . . 72

4.4 Simulated gas saturation (no salinity). . . . . . . . . . . . . . 73

4.5 Simulated dissolved $\mathrm{CO}_{2}$ mass fraction (no salinity). . . . . . . . . 73

4.6 Simulated pressure (10 wt.- $\%$ salinity $) \ldots \ldots$. . . . . . . . . 76

4.7 Simulated gas saturation (10 wt.- $\%$ salinity). . . . . . . . . . . 76

4.8 Simulated dissolved $\mathrm{CO}_{2}$ mass fraction (10 wt.- \% salinity). . . . . . . 77

4.9 Simulated solid salt saturation (10 wt.- \% salinity). . . . . . . . . . . 77

4.10 Simulated solid salt saturation using a finer grid near the injection well 78

4.11 Schematic representation of the Sleipner storage system (From Zweigel et al. [2000]). . . . . . . . . . . . . . . . . . . . . . 79

4.12 Simplified 2-D vertical section of the Sleipner storage system . . . . . 80

4.13 Simulated gas saturation (top) and dissolved $\mathrm{CO}_{2}$ (bottom) after 1 year. 83

4.14 Simulated gas saturation (top) and dissolved $\mathrm{CO}_{2}$ (bottom) after 2 years. 84

4.15 Dissolved $\mathrm{CO}_{2}$ in the aqueous phase contours at $t=100,300,500,800$, 1200, and 1800 years . . . . . . . . . . . . . . 89

4.16 Simulated mass fraction ratio as free gaseous phase. Diffusion curve is obtained from numerical simulations that ignore the effect of $\mathrm{CO}_{2}$ content in aqueous phase density. . . . . . . . . . . . . . 90

$5.1 \mathrm{CO}_{2}$ viscositiy and viscosity ratio $\frac{\mu_{\mathrm{H}_{2} \mathrm{O}}}{\mu_{\mathrm{CO}_{2}}}$ profile. . . . . . . . . . . 93

5.2 Viscous fingering in rectilinear flow . . . . . . . . . . . . . . 95

5.3 Schematic of flow system. . . . . . . . . . . . . . . . . . . . . . . . . . . . 97

5.4 Fractional Flow Function. . . . . . . . . . . . . . . . . . . . . 99

5.5 Buckley-Leverett solution for mobilities. Symbols represent the TOUGH2EOS3f solution. . . . . . . . . . . . . . . . 100

5.6 Buckley-Leverett solution for gas saturation. Symbols represent the numerical solution with front tracking (TOUGH2-EOS3f). . . . . . . 100

5.7 Stability of immiscible displacement for different relative permeability functions. Curves $1,2,3,4$, and 5 refer to values of $\mathrm{m}=0.9,0.8,0.7$, $0.6,0.5$ respectively in the van Genuchten [1980] relative permeability function. . . . . . . . . . . . . . . . . . 
5.8 Mobility shock profile for different relative permeability functions. Curves $\mathrm{a}, \mathrm{b}$, and $\mathrm{c}$, refer to values of $\lambda=0.7,0.8$ and 0.9 respectively in the van Genuchten [1980] relative permeability function. . . . . . . . . . . . 103

5.9 Viscous fingers are triggered (b) when a modified permeability field is used (a). No fingering occurs otherwise (c) . . . . . . . . . . . . . . 107

5.10 Grid Convergence (a) 15,480 (b) 61,920 (c) 141,120 Grid Blocks. Lines represent gas saturation contour levels with 0.02 increments, from $S_{g}=$ 0.02 (right) to $S_{g}=0.10$ (left). . . . . . . . . . . . . . . . 108

5.11 Coarse grid (30,000 blocks, scalar machine, top) and fine grid (203,200 blocks, massively parallel scheme, bottom) simulations of viscous fingering. . . . . . . . . . . . . . . . . . . . . . . 109

5.12 No-flow boundary conditions effect. (a) top and bottom no-flow boundaries. (b) top and bottom boundaries connected. . . . . . . . . . . . . 111

5.13 No flow boundary conditions (left) can be eliminated by creating artificial 2-D horizontal cylinder type grids (right). . . . . . . . . . . . . 111

5.14 Finger Wave-Length. . . . . . . . . . . . . . . . . . . . . . 114

5.15 Finger Growth. Lines represent frontal gas saturation contour levels $\left(0.08 \geq S_{g} \geq 0.02\right)$ at $T=\left(1.0 \times 10^{7}, 2.0 \times 10^{7}, 3.0 \times 10^{7}\right)$ sec. . . . 115

5.16 High-resolution simulation results for finger growth. . . . . . . . . 115

5.17 Viscous fingering predictions obtained from ECO2 (top) and EOS3 (bottom). . . . . . . . . . . . . . . 117 



\section{List of Tables}

2.1 Triple and critical point parameters for $\mathrm{CO}_{2}$. . . . . . . . . 15

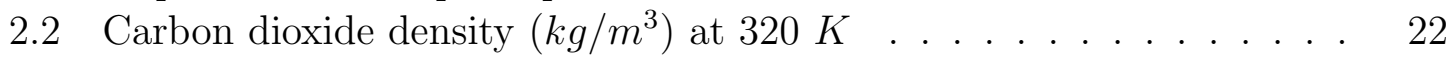

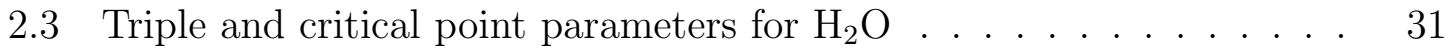

2.4 Solubility of carbon dioxide in brines (mole fractions) . . . . . . . . 45

3.1 List of all dependent variables . . . . . . . . . . . . . . . 55

3.2 Short description of subroutines in Figure $3.3 \ldots \ldots 6$

4.1 Hydrologic parameters for one-dimensional radial flow simulations . . 71

4.2 Hydrologic parameters for two-dimensional layered brine formation . 81

4.3 Hydrologic parameters for convective mixing simulations . . . . . . . 87

5.1 Fluid Property Modules . . . . . . . . . . . . . . . . . 105 



\section{Acknowledgments}

Many people have contributed in the initiation, development and completion of this dissertation, each in their own special way. Without their personal contributions this work would not have been possible.

I would like to express my deep gratitude and sincere appreciation to Dr. Karsten Pruess for his support, encouragement, and guidance throughout the course of this study. He has been a consistent source of knowledge and inspiration. Dr. Pruess provided me great deal of freedom to explore different ideas, and at the same time gave me constructive suggestions and guidance. It was a distinct privilege for me to work with him.

I would also like to thank Prof. Nicholas Sitar for his advice during the development of this dissertation. Prof. Sitar was a great teacher and example to me. I owe him many thanks for all the discussions, sparkling scientific enthusiasm and friendship. I am also thankful to Professors Kent Udell and Tadeusz Patzek for taking an active interest in my work and serving on my dissertation committee.

I am indebted, directly or indirectly, to many scientists and in general to the staff of Berkeley Lab's Earth Sciences Division for creating a flexible and enthusiastic working environment in which I was able to develop my research. This work benefited from discussions with Tianfu Xu, Nicolas Spycher, Sally Benson and Curt Oldemburg. Special thanks are due to Keni Zhang for his help adapting the parallel version of TOUGH2 to some of the viscous fingering simulations presented in this work. I am grateful to Jonathan Ennis-King of CSIRO, Australia, for the fruitful discussions about numerical simulation of carbon dioxide disposal in aquifers.

Needless to say, I am grateful to all my fellow students. I am especially thankful to David Mays, Tai-Sheng Liou and Mike Kowalsky for being my officemates, and for sharing my joys and frustrations. Thanks also go to my friend Ruben Juanes for many invigorating discussions and the delightful afternoon coffee breaks. My years 
at Cal would not have been as enjoyable without the friendship of Paolo Gardoni and Gian-Marco Pizzo.

Now more than ever, I remember my faculty, colleagues, and friends from Colombia and Colorado. I am particularly grateful to Mario Diaz-Granados, who imparted to me a fascination for the field hydrology, and encouraged me to continue my education in the United States. I am thankful to the people at GeoIngenieria for helping me mature as an engineer. I also want to thank Juan Manuel Martinez, Carlos Molano, and the people at COLFUTURO, who have helped me in so many different ways. Finally, at the University of Colorado, Prof. Tissa Illangasekare stirred me to continue doing research in hydrogeology and multiphase flow in porous media.

I am particularly thankful to the Organization of American States (Washington, D.C.) and the International House at UC Berkeley, whose fellowship and scholarship provided funding during my first year at Berkeley. Their financial support is gratefully acknowledged.

Throughout my life and particularly during my years in graduate school, my parents and siblings were an ongoing source of unconditional love and support. Every member of my family made sacrifices to see me succeed. Above all, I would like to thank my brother Gerardo, whose calm presence, support, and love, were invaluable during my years as a graduate student. Finally, I would like to express my immeasurable gratitude to Laura, my "chulis" during all these years at Berkeley, who helped me understand the simplicity of life and lent an endless supply of support, joy and love.

This work was supported by the US Department of Energy through the Office of Basic Energy Sciences and through the National Energy Technology Laboratory (NETL) under Contract No. DE-AC03-76SF00098. 


\section{Chapter 1}

\section{Introduction}

This dissertation addresses geohydrologic issues related to carbon dioxide $\left(\mathrm{CO}_{2}\right)$ disposal into saline aquifers. Special emphasis is given to the physics of fluid flow in brine formations, injection behavior and long term containment of supercritical $\mathrm{CO}_{2}$. Issues regarding reactive chemistry (rock-fluid interactions and chemical modeling) and stress change, although considered very important, are beyond the scope of this work. This introduction begins with a brief overview of carbon sequestration in geologic formations and the motivation behind it. The specific objectives of the dissertation are listed in Section 1.2. The scope and brief outline of the dissertation is presented at the end.

\subsection{Background and Motivation}

Carbon dioxide $\left(\mathrm{CO}_{2}\right)$ is a greenhouse gas, whose release into the atmosphere from combustion of fossil fuels contributes to global warming. Trace gases in the atmosphere like carbon dioxide, methane and water vapor can trap infrared radiation escaping into space. Without such naturally occurring gases in our atmosphere, the Earth's average temperature would be $-18{ }^{\circ} \mathrm{C}$ instead of the comfortable $+15{ }^{\circ} \mathrm{C}$ 
it is today. However, problems may arise when the atmospheric concentration of greenhouse gases increases. Rising global temperatures are expected to raise sea level, and change precipitation and other local climate conditions. Changing regional climate could alter forests, crop yields, and water supplies. It could also threaten human health and many types of ecosystems.

Because of its relative abundance compared to other greenhouse gases, $\mathrm{CO}_{2}$ is by far the most important, being responsible for about $64 \%$ of the enhanced "greenhouse effect" (United Nations Framework Convention on Climate Change, UNFCCC [2002]). The rise of carbon dioxide concentration in the atmosphere since the beginning of the industrial revolution has been substantial. $\mathrm{CO}_{2}$ concentration has increased from around 280 parts per million by volume (ppmv) in the $18^{\text {th }}$ century to over 360 ppmv by 1997, with half this increase having occurred since the mid 1960's. Currently the burning of fossil fuels provides about $85 \%$ of the world's energy. Energy burned to run cars and trucks, heat homes and businesses, and power factories is responsible for about $80 \%$ of society's carbon dioxide emissions. Although the development of more efficient and alternative energy systems will lead to a reduction in greenhouse emissions, it is inevitable that the burning of fossil fuels will continue to provide a considerable proportion of the world's energy well into the $21^{s} t$ century.

Carbon dioxide sequestration appears to be an important potential method by which emissions into the atmosphere can be reduced. Before $\mathrm{CO}_{2}$ gas can be sequestered it must be captured from point sources such as power plants. In addition to carbon dioxide, oxygen and water vapor, flue gases originating from the combustion of fossil fuels contain also $\mathrm{N}_{2}, \mathrm{NO}_{x}, \mathrm{SO}_{x}$, and noble gases. Exhaust gas composition and $\mathrm{CO}_{2}$ capture options depend on the power generation scheme considered. Currently, the main technologies used to generate power from fossil fuels are [IEA, 2001]:

1. Pulverized coal-fired steam cycle. This represents the most commonly available technology. Pulverized coal is burned in a boiler producing high pressure steam, 
which is then passed through a steam turbine, generating electricity.

2. Natural gas combined cycle. Natural gas is burned in gas turbine, generating electricity. Then, the hot exhaust gas is fed to a boiler which generates steam, which is passed through a steam turbine, generating more electricity.

3. Integrated gasification combined cycle (IGCC). In this type of plant, fuel (residual oil, coal or a combination of fossil fuels) is reacted with oxygen and steam in a gasifier to produce a fuel gas that is burned to generate power in a gas turbine combined cycle.

$\mathrm{CO}_{2}$ is only a small part of the flue gas stream emitted to atmosphere by a power plant. For pulverized coal fired, coal fired IGCCC, and natural gas combined cycle power stations the $\mathrm{CO}_{2}$ concentration in flue gas is approximately 14, 9, and $4 \%$ by volume, respectively. The small concentration of $\mathrm{CO}_{2}$ in flue gas means that a large volume of gas has to be handled, resulting in large and expensive equipment. According to Herzog et al. [1997] the "energy penalty" for capturing $\mathrm{CO}_{2}$ with today's technology would be between 15 and 25\%. For example, the output of a $500 \mathrm{MW}_{e}$ coal-fired power plant may be reduced to between 375 and $425 \mathrm{MW}_{e}$. If there is no substantial improvement in technology it may be more economical to inject mixtures that include major constituents other than $\mathrm{CO}_{2}$. Nevertheless, even with these limitations, fossil fuel-fired power plants are ideal candidates for carbon capture since they produce one-third of United States $\mathrm{CO}_{2}$ emissions in the generation of electricity for residential, commercial, and industrial customers [DOE, 1999].

The oceans represent possibly the largest potential sink for sequestration of anthropogenic $\mathrm{CO}_{2}$ emissions. However, ocean disposal involves physical-chemical processes that are not well understood, high cost, and present technical difficulties and possible major environmental impacts. Additionally, ocean circulation processes may bring legal, political and international limitations to large scale direct injection of 
$\mathrm{CO}_{2}[\mathrm{Bachu}, 2000]$.

Carbon dioxide disposal in geological formations represents another possible large capacity sink. Because most power plants are not close to the oceans, geological disposal of $\mathrm{CO}_{2}$ actually represents an attractive alternative. The options for underground storage include:

- Active and depleted oil and gas reservoirs

- Deep aquifers, including saline formations

- Deep coal seams and coal-bed methane formations

In active and partially depleted oil reservoirs carbon dioxide can be used to enhance oil production by two primary mechanisms. First, $\mathrm{CO}_{2}$ gas displaces oil and brine, which are subsequently pumped from the wells. Second, injected $\mathrm{CO}_{2}$ dissolves in the oil, leading to a reduction in viscosity and swelling of the oil, making it flow more easily and leading to enhanced production. Similarly, coal formations provide an opportunity to simultaneously sequester $\mathrm{CO}_{2}$ and increase production of natural gas. Coal has a greater affinity for $\mathrm{CO}_{2}$ than methane $\left(\mathrm{CH}_{4}\right)$ and it is stored by adsorption.

Sequestration of $\mathrm{CO}_{2}$ in deep saline aquifers does not provide value added products, but it has other advantages. First, the estimated storage capacity of saline aquifers is very large. Second, most existing large $\mathrm{CO}_{2}$ point sources are within easy access of a saline formation. From an engineering perspective, the main issues for $\mathrm{CO}_{2}$ disposal in aquifers relate to (1) the rate at which $\mathrm{CO}_{2}$ can be disposed, (2) the available storage capacity (ultimate $\mathrm{CO}_{2}$ inventory), (3) the presence of caprock of low permeability, and the potential for $\mathrm{CO}_{2}$ leakage through imperfect confinement, which may be natural or induced, (4) identification and characterization of suitable aquifer formations and caprock structures, (5) uncertainty and possibility of failure 
due to incomplete knowledge of subsurface conditions and processes, and (6) corrosion resistant materials to be used in injection wells and associated facilities [Pruess et al., 2003].

There are three ways in which $\mathrm{CO}_{2}$ can be sequestered in brine aquifers. First, $\mathrm{CO}_{2}$ can be trapped under a low-permeability caprock, similar to the way natural gas is trapped in reservoirs and stored in aquifers. This mechanism called hydrodynamic trapping relies on the physical displacement of pore fluids. In the second mechanism, pore fluids can accumulate dissolved $\mathrm{CO}_{2}$ through aqueous or solubility trapping. In the third type of trapping, dissolved $\mathrm{CO}_{2}$ reacts with divalent cations to form carbonate mineral precipitates. This mechanism called mineral trapping offers the advantage of increasing $\mathrm{CO}_{2}$ sequestration capacity while also rendering the $\mathrm{CO}_{2}$ immobile for long time scales.

In order to avoid adverse effect from $\mathrm{CO}_{2}$ separating into liquid and gas phases in the injection line, geologic disposal of $\mathrm{CO}_{2}$ would be made at supercritical pressures [Pruess and García, 2002]. The critical point of $\mathrm{CO}_{2}$ is at $P_{\text {crit }}=73.82$ bar, $T_{\text {crit }}=$ $31.04{ }^{\circ} \mathrm{C}$ [Vargaftik et al., 1996], so that minimum aquifer depths of approximately 800 $m$ would be required to sustain a supercritical pressure regime. Large-scale injection of $\mathrm{CO}_{2}$ will induce a variety of coupled physical and chemical processes, including:

1. Multiphase fluid flow. The process of $\mathrm{CO}_{2}$ injection into saline aquifers can be classified as immiscible displacement of an aqueous phase by a less dense and less viscous gas phase. Therefore, the displacement of aquifer water by $\mathrm{CO}_{2}$ is subject to hydrodynamic instabilities, including viscous fingering and gravity override.

2. Fluid pressurization and changes in effective stress. Injection of $\mathrm{CO}_{2}$ into an aquifer will produce an increase in pore pressure, which, in turn will alter the effective stress state leading to permeability and porosity variations. A suffi- 
ciently large change in the stress field could cause failure by allowing $\mathrm{CO}_{2}$ to leak through fractured rock, and may give rise to seismicity as well [Rutqvist and Tsang, 2002].

3. Solute transport. Dissolution of $\mathrm{CO}_{2}$ in the aqueous phase takes place in two steps. First, $\mathrm{CO}_{2}$ transfers across the gas-water interface to become carbonic acid. Second, there is a rapid dissociation into bicarbonate ion $\mathrm{HCO}_{3}^{-}$and $\mathrm{H}^{+}$. Solute transport in porous media can then be modeled by traditional approaches [de Marsily, 1986].

4. Chemical reactions between fluids and formation minerals. Carbon dioxide can react directly or indirectly with certain minerals in a geologic formation leading to the precipitation of secondary carbonates (mineral trapping). This results in a porosity decrease with an associated potential for significant decrease in permeability [Xu et al., 2003].

Early engineering knowledge for sequestering $\mathrm{CO}_{2}$ in geologic formations is based on many years of proven experience with injecting supercritical $\mathrm{CO}_{2}$ for enhanced oil recovery [Blunt et al., 1993; Orr and Taber, 1984]. Today, most commercial petroleum engineering simulators can be used to model phase-partitioning and flow behavior during injection of $\mathrm{CO}_{2}$ for enhanced oil recovery. However, special emphasis is given to the oil- $\mathrm{CO}_{2}$ behavior treating the water- $\mathrm{CO}_{2}$ system with relatively rough approximations. Significant experience with flow systems which involve water, salt and $\mathrm{CO}_{2}$ exists in geothermal reservoir engineering [Andersen et al., 1992; Battistelli et al., 1997]. Nevertheless, geothermal simulators address higher temperatures and lower $\mathrm{CO}_{2}$ partial pressures than would be encountered in aquifer disposal of $\mathrm{CO}_{2}$ [Pruess and García, 2002]. There is also considerable experience with natural gas storage in aquifers in the midwestern U.S states of Illinois and Indiana and salt caverns in Texas [DOE, 2003; Katz and Lee, 1990]. Although the idea of injecting $\mathrm{CO}_{2}$ 
into brine aquifers has been around for many years, the Sleipner Vest project in the Norwegian sector of the North Sea is the only practical example of $\mathrm{CO}_{2}$ disposal into an aquifer [Korbøl and Kaddour, 1995]. There is clearly a need for engineering tools that will allow us to develop a systematic, rational and mechanistic understanding of the coupled physical and chemical processes that would be induced by injection of $\mathrm{CO}_{2}$ into aquifers. As a first step, a better knowledge of fluid dynamics processes can be accomplished by means of conceptual, mathematical, and numerical models that are based on these essential components:

- Simple, yet accurate representations and models for predicting the overall thermophysical behavior of the system $\mathrm{CO}_{2}-\mathrm{H}_{2} \mathrm{O}-\mathrm{NaCl}$ in a geologic carbon sequestration context.

- Appropriate mathematical representation and correct numerical implementation of multiphase flow in porous media.

- Application of simulation capabilities to suitable and relevant test problems.

- Possibility for easy integration of numerical simulation capabilities for multiphase flow with geochemical and geomechanical simulators.

\subsection{Objectives}

The aim of this dissertation is to provide a scientific basis for the possible disposal of $\mathrm{CO}_{2}$ into deep saline aquifers, as an alternative to release into the atmosphere. Our final goal is to obtain a better understanding of $\mathrm{CO}_{2}$ disposal into brine formations to assess its technical feasibility, identify favorable and unfavorable conditions and provide a tool for engineering design and monitoring. In order to accomplish our proposed goal, the major elements of this research work was divided into three parts: 
1. Develop an understanding of thermodynamics and thermophysical properties of $\mathrm{CO}_{2}$ and mixtures of $\mathrm{CO}_{2}$ with saline waters, and implement suitable numerical formulations

2. Incorporation of the fluid property description into a module for the TOUGH2 simulator.

3. Application of simulation capabilities for modeling $\mathrm{CO}_{2}$ injection in brine aquifers.

\subsection{Overview}

This dissertation is organized in six chapters. A detailed description of the thermophysical properties of pure carbon dioxide, water, and aqueous solutions of $\mathrm{CO}_{2}$ and $\mathrm{NaCl}$ is presented in Chapter 2. Phase equilibria models for the system carbon dioxide-water-salt are also discussed in this chapter. Additionally, this Chapter 2 includes a discussion of solubility models and ends by providing recommendations for numerical correlations for stable and robust simulations.

The numerical simulator for carbon sequestration into saline aquifers, TOUGH2/ECO2 is presented in Chapter 3. The mathematical formulation, space and time discretization and solution method presented there follow the general methodology of TOUGH2 [Pruess et al., 1999]. Details of ECO2 module operativity and capabilities are also presented in Chapter 3.

In Chapter 4, the basic problem of $\mathrm{CO}_{2}$ injection into a radially symmetric brine aquifer is used to validate the results of TOUGH2/ECO2. Because the simplified radial flow admits a similarity solution, this problem is used to check that the governing equations of the mathematical model have been correctly implemented, and that the numerical simulation confirms the similarity property. Two additional, more complex flow problems, are also considered in this chapter. One represents the $\mathrm{CO}_{2}$ injection 
project at the Sleipner Vest Field and the other addresses issues regarding enhanced $\mathrm{CO}_{2}$ dissolution by convective mixing during long term containment of carbon dioxide. The purpose of Chapter 5 is to provide better understanding of the onset of viscous fingering during immiscible displacement in porous media. Particular emphasis is placed on the flow instabilities that may occur during injection of $\mathrm{CO}_{2}$ into saline aquifers. The analysis is based on two-dimensional (2D) high-resolution numerical simulations carried out on scalar machines, and on an IBM RS/6000 SP (a distributedmemory parallel computer with 6080 processors) with a parallelized version of TOUGH2.

Chapter 6 contains the summary of the main results and suggestions for new research directions. 



\section{Chapter 2}

\section{Thermophysical properties of fluid mixture components}

\section{$2.1 \quad$ Introduction}

A detailed description of the thermophysical properties of pure carbon dioxide, water, and aqueous solutions of $\mathrm{CO}_{2}$ and $\mathrm{NaCl}$ is presented in this chapter. Thermophysical properties of the individual fluids, as wells as of fluid mixtures, have been extensively studied and there is a considerable amount of literature on this subject. The main purpose of the work presented here is to provide simple, but accurate representations and models for predicting the overall thermophysical behavior of the system $\mathrm{CO}_{2}-\mathrm{H}_{2} \mathrm{O}-\mathrm{NaCl}$ in a geologic carbon sequestration context. The final aim is to incorporate the selected models into the numerical simulator TOUGH2 for modeling carbon sequestration into saline aquifers.

The state of equilibrium for a given system is characterized by a well defined number of intensive properties. From the Gibbs' phase rule [Gibbs, 1876, 1878] it follows that the number of independent variables that must be arbitrarly fixed is 
given by:

$$
F=2+n-r
$$

where $n$ is the number of chemical species, $r$ is the number of phases, and $F$ is called the degrees of freedom of the system. Equation 2.1 provides the fundamental basis for a quantitative description of any thermodynamic system. For a system with three species $\left(\mathrm{CO}_{2}, \mathrm{H}_{2} \mathrm{O}\right.$ and $\left.\mathrm{NaCl}\right)$ the number of independent variables required to characterized the thermophysical system is given by $F=5-r$.

This chapter is organized as follows. In Sections 2.2 and 2.3 the models for thermophysical properties of pure carbon dioxide and pure water, respectively, are presented. In Section 2.4 the phase behavior and solubility models in the $\mathrm{CO}_{2}-\mathrm{H}_{2} \mathrm{O}$ system are described. A discussion of thermophysical properties of fluid mixtures is presented in Section 2.5. Salinity effects on $\mathrm{CO}_{2}$ solubility are discussed in Section 2.6. Finally, Section 2.7 provides some concluding remarks and recommendations.

\subsection{Thermophysical properties of pure $\mathrm{CO}_{2}$}

During the first part of the twentieth century the use of $\mathrm{CO}_{2}$ as the working fluid in refrigerators initiated intensive research into its thermodynamic properties at low temperatures and on the saturation line. For a long time, definite prospects for the technological use of $\mathrm{CO}_{2}$ at high temperatures and pressures were not apparent and research into its thermophysical properties was mainly of scientific interest. In the mean time, the relatively moderate values of its critical parameters facilitated research into properties in the critical region [Vukalovich and Altunin, 1968]. The push for accurate representations of $\mathrm{CO}_{2}$ thermophysical properties was driven by its intended application as a coolant in atomic power stations in the early 1950's 
(Supercritical $\mathrm{CO}_{2}$ ). Furthermore, the interest on carbon dioxide properties grew as it proved to be a powerful solvent as a supercritical fluid and an excellent tool for enhanced oil recovery. In other areas of the earth sciences, interest is based on the widespread natural occurrence of carbon dioxide. Geophysical investigations require thermodynamic data for $\mathrm{CO}_{2}$ at high pressures and temperatures. More recently, the debate on the role of $\mathrm{CO}_{2}$ in the greenhouse effect and global warming sparked the interest on its thermophysical properties from low temperature (hydrate formation, ocean carbon sequestration) and low pressure (atmospheric) to intermediate pressures and temperatures (geological carbon sequestration).

\subsubsection{Volumetric properties}

The main fluid properties needed in the governing mass balance equations for numerical simulation of flow in porous media are density and viscosity of individual phases. This requirement explains the relevance of accurate Pressure-VolumeTemperature (PVT) relationships. In addition, thermodynamics properties such as internal energy, enthalpy and fugacity are often evaluated from volumetric correlations. Furthermore, recent accurate correlations for viscosity require density as an input (Fenghour et al. [1998] and Rah and $\mathrm{Eu}$ [2001]). Vapor pressure measurements for a pure substance lead to the pressure-vs.-temperature curves such as shown by the sublimation and vapor-saturation lines for $\mathrm{CO}_{2}$ in Figure 2.1. Also shown in Figure 2.1 is the line representing the fusion or melting curve for pure $\mathrm{CO}_{2}$. The lines in the pressure-temperature (PT) diagram represent conditions where two phases coexist (i.e. $r=2, F=1$ in Equation 2.1) and are the boundaries for single phase regions (i.e. $r=1, F=2$ ). All three lines meet at the triple point (invariant point with no degrees of freedom, $F=0$ ). The vaporization curve ends at the critical point, which corresponds to the highest pressure and highest temperature at which a pure chemical species can exist in vapor-liquid equilibrium. Critical and triple point data 
for $\mathrm{CO}_{2}$ are presented in Table 2.1 .

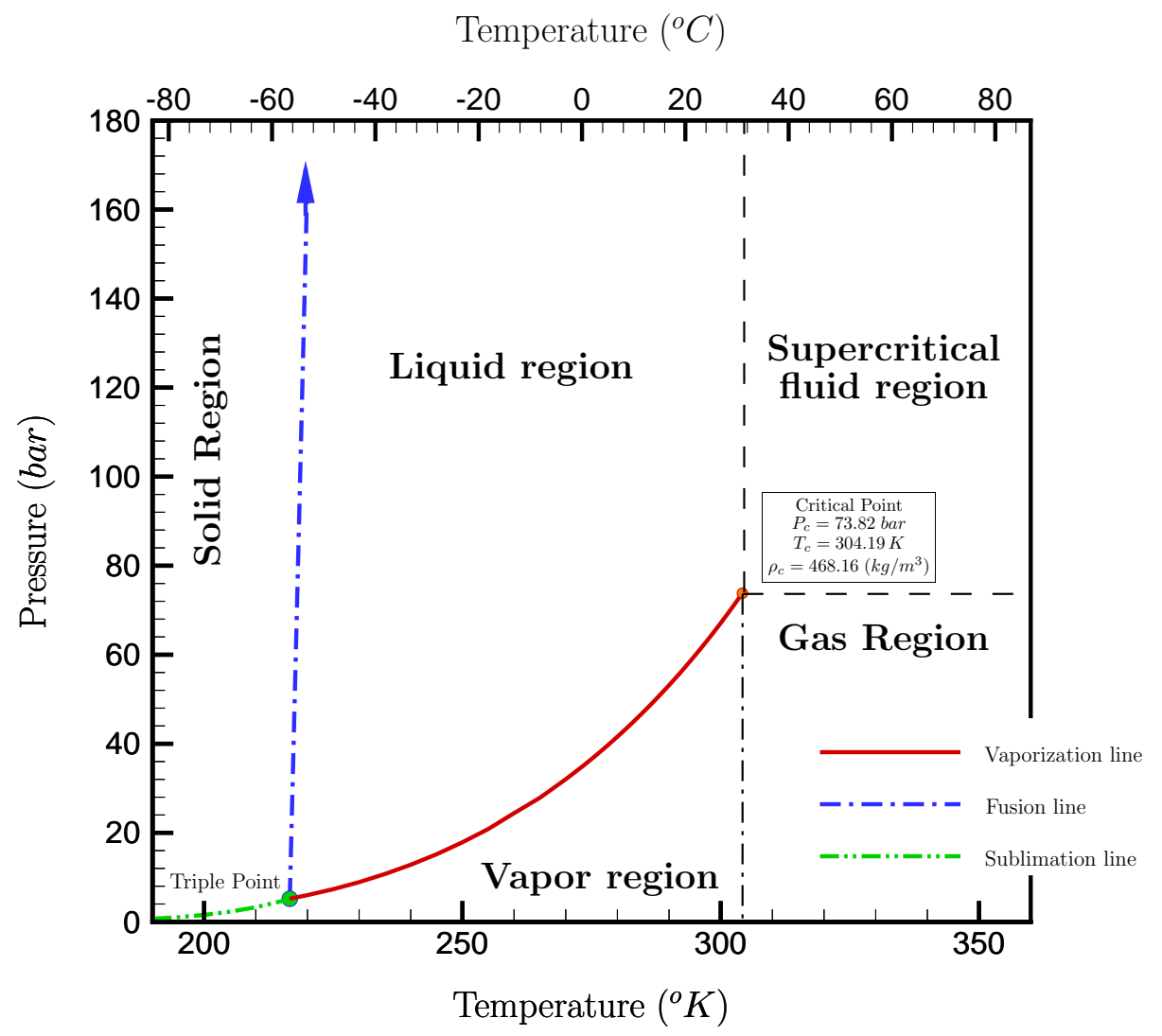

Figure 2.1: Pressure-temperature diagram for pure carbon dioxide

Figure 2.1 does not provide any information about volume properties, it only displays the boundaries between phases on a PT diagram. On a pressure-volume (PV) diagram these boundaries become areas, i.e. regions where two phases coexist. Figure 2.2 shows the different phase regions on a pressure versus volume diagram for carbon dioxide, with three isotherms superimposed. Lines for subcritical temperatures (for example $T_{1}=290 \mathrm{~K}$ in Figure 2.2) consist of three segments. The horizontal segment represents all the possible mixtures of liquid and vapor equilibrium. The two end points indicate, $100 \%$ liquid at the left, and $100 \%$ vapor at the right. The locus of these end points defines a bell shape curve under which the liquid-vapor region lies. Subcooled liquid and superheated vapor lie to the left and right, respectively. 
Table 2.1: Triple and critical point parameters for $\mathrm{CO}_{2}{ }^{\mathrm{a}}$

\begin{tabular}{lcc}
\hline & \multicolumn{2}{c}{ Carbon Dioxide $-\mathrm{CO}_{2}$} \\
& Triple Point & Critical Point \\
\hline $\mathrm{P}(\mathrm{bar})$ & 5.18 & 73.82 \\
$\mathrm{~T}(\mathrm{~K})$ & 216.55 & 304.19 \\
$\rho_{v}\left(\mathrm{~kg} / \mathrm{m}^{3}\right)$ & 13.80 & 468.16 \\
$\rho_{l}\left(\mathrm{~kg} / \mathrm{m}^{3}\right)$ & 1179.25 & 468.16 \\
\hline
\end{tabular}

${ }^{a}$ values reported by Vargaftik et al. [1996]

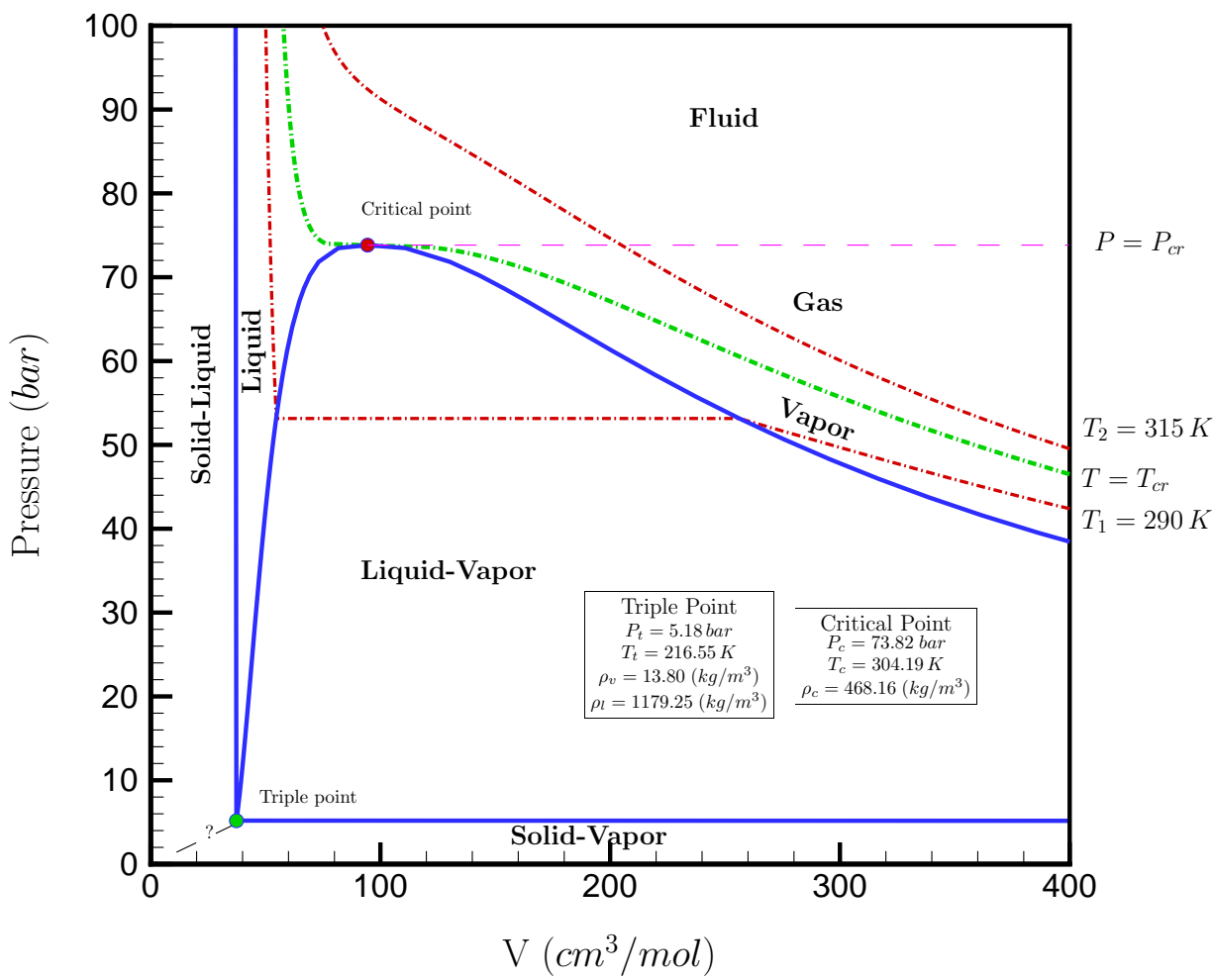

Figure 2.2: Pressure-volume diagram for pure carbon dioxide 


\subsubsection{Analytical equations of state}

For single phase regions a relation connecting pressure, volume, and temperature exists and may be expressed by a functional equation:

$$
f(P, V, T)=0
$$

Equations of the type 2.2 are denoted Equation of State as they relate the state parameters pressure, molar or specific volume, and temperature. The simplest equation of state is the law relating pressure, temperature and volume for a gas with molecules of zero size and without intermolecular forces, also known as the ideal gas law

$$
P V=R T
$$

where $R=83.144 \mathrm{bar} \cdot \mathrm{cm}^{3} /(\mathrm{mol} \cdot K)$ is the gas constant. The compressibility factor, $Z=\frac{P V}{R T}$, is often used to expressed the nonideality of a gas. For an ideal gas $Z=1.0$, for real gases, $Z$ is usually less than 1 , except for very large pressures. The compressibility factor is often correlated as a function of reduced temperature $T_{r}$ and reduced pressure $P_{r}$.

$$
Z=f\left(T_{r}, P_{r}\right)
$$

where $T_{r}=T / T_{c}$ and $P_{r}=P / P_{c} . T_{c}$ and $P_{c}$ are the critical temperature and pressure, respectively. Equation 2.4 is the basis for the two-parameter principle of corresponding states: All fluids, when compared at the same reduced temperature and reduced pressure, have approximately the same compressibility factor, and all deviate from ideal-gas behavior to about the same degree [Smith et al., 2001]. For simple 
fluids (non-polar and spherical molecules), two-parameter corresponding state correlation gives volumetric properties with an error of 4-6\%. For the case of $\mathrm{CO}_{2}$ (flat molecule) the two-parameter correlation gives poor approximations. Improvements can be achieved by introducing a third corresponding-state parameter that accounts for molecular structure; the most common parameter used is the acentric factor $\omega$ [Pitzer, 1995].

A better description of volumetric properties can be achieved using analytical equations of state through algebraic relations between pressure, temperature, and molar volume. These relations can be very simple in form (perfect gas law), but also, when improved accuracy is required, they can be computationally complex.

\subsubsection{Cubic equations of state}

Cubic equations of state deserve special attention. In fact, polynomial equations that are cubic in molar volume had proved to be the simplest equations capable of representing both liquid and vapor behavior. The first practical equation was introduced by J.D. van der Waals in 1873:

$$
P=\frac{R T}{V-b}-\frac{a}{V^{2}}
$$

where $a$ and $b$ are positive parameters; when they are zero, Equation 2.5 becomes the ideal-gas Equation 2.3. $b$, with units of $\left[\mathrm{m}^{3} / \mathrm{mol}\right]$ represents the excluded volume effect, whereas $a$, with units of $[$ bar $]$ reflects attractive force between molecules. A more general cubic equation of state is given by:

$$
Z^{3}-A^{*} Z^{2}+B^{*} Z-C^{*}=0
$$

where $A^{*}, B^{*}$, and $C^{*}$ are coefficients function of $a$ and $b$. All cubic EOS can be 
written as Equation 2.6 with different functions for $a, b, A^{*}, B^{*}$, and $C^{*}$ [Reid et al., 1987]. Cubic equations of state are easy to use and their solutions provide a first approximation to molar volume, thus to density of pure fluids. Furthermore, these equations can be easily manipulated to obtain other derived properties such as enthalpy and fugacity. In fact, these equations are used more often for the latter than for estimation of the density. Whereas, more complicated equations of state are used for accurate representation of the density. In section 2.2.2.1, the applicability of these equations to predict density as well as other thermophysical properties is illustrated.

\subsubsection{Density correlations for carbon dioxide}

In recent years a great amount of work has been carried out in reviewing and acquiring new thermophysical data for pure carbon dioxide. The result of this effort is the availability of accurate correlations of $\mathrm{CO}_{2}$ PVT data covering a wide range of temperatures and pressures.

Accurate correlations of carbon dioxide PVT data are available from several published scientific papers and monographs. Among them, the ones proposed by Altunin [1975], Angus et al. [1976], Span and Wagner [1996] and Mäder and Berman [1991] are the most broadly accepted and used. Altunin's correlation, given in a similar form to the virial equation, relates the compressibility factor $Z$ with the two independent variables, reduced density $\rho_{r}=\rho / \rho_{c}$ and reduced temperature $T_{r}=T_{c} / T$. The correlation developed by Altunin and coworkers is the result of extensive studies of the PVT properties of $\mathrm{CO}_{2}$ carried out in the former Soviet Union. An earlier version of this correlation [Altunin and Gadetskii, 1971] has been successfully used in other parts of the world and partially incorporated in the International Union of Pure and Applied Chemistry (IUPAC, Angus et al. [1976]) thermodynamic tables of carbon dioxide. The complete equation of state used in the calculation of the IUPAC tables is a combination of Altunin and Gadetskii [1971] correlation outside the critical region 
and the correlations by Schofield [Schofield, 1969; Schofield et al., 1969] for the region within $5 K$ of the critical point. The most recent and widely used equation of state is due to Span and Wagner [1996]. In their paper, the authors provided an excellent review of other available equations of state and the reason why they think these correlations could be substantially improved. Span and Wagner [1996] presented an equation of state explicit in the Helmholtz energy, $A$, with two independent variables $\rho_{r}$ and $T_{r}$. All the other thermodynamics properties for pure carbon dioxide were obtained by combining derivatives of the correlation for dimensionless molar Helmholtz energy $\widetilde{A}=A /(R T)$. Finally, another source for thermodynamics properties of carbon dioxide are the PVT tables reported by Vargaftik et al. [1996] whose compilation is based on correlations originally reported by Kessel'man and Kotloarevskii [1965].

Figure 2.3 shows the density contours for $\mathrm{CO}_{2}$ calculated using the correlations developed by Altunin [1975] as implemented by V. I. Malkowsky of IGEM, Moscow (private communication). It is worth to mention here, that in the temperature and pressure range considered for carbon sequestration, $\mathrm{CO}_{2}$ is less dense than $\mathrm{H}_{2} \mathrm{O}$ by a factor of 2.5. As will be explained in Chapter 3, for a robust and efficient calculation of density as well as other thermophysical properties we used a tabular equation of state calculated from the correlations of Altunin [1975]. We have used the data as reported by Vargaftik et al. [1996] and by Span and Wagner [1996] to evaluate the accuracy of the Altunin's correlation. The results shown in Figure 2.4 demonstrate that in the range of interest the agreement is excellent (error below 1\%).

Alternatively, densities can be calculated using the cubic EOS described in the previous section. For that purpose Equation 2.6 is solved for $Z$ and the molar density is evaluated as:

$$
\rho=\frac{P}{R T Z}
$$

Cubic equations of state have three molar volume $V$ roots (or three compressibility 


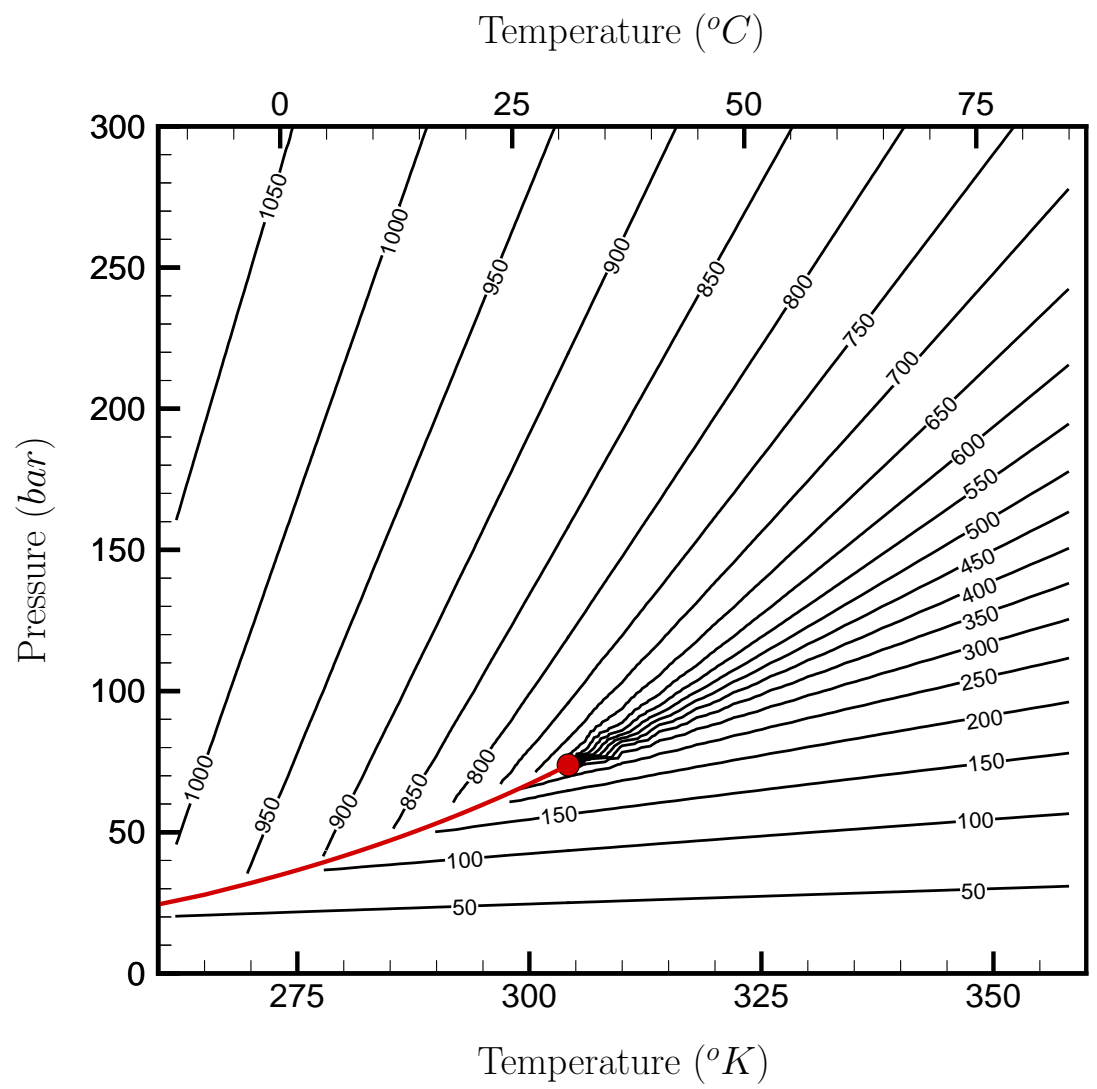

Figure 2.3: Calculated $\mathrm{CO}_{2}$ density $\left(\mathrm{kg} / \mathrm{m}^{3}\right)$ using the correlations of Altunin [1975]

factor $Z$ ), of which two may be complex. Physically meaningful values of $V$ are always real, positive, and greater than the constant $b$. For $T \geq T_{c}$ only one real root exists, whereas below the critical point Equation 2.6 can yield more than one volume value [Spycher et al., 2003]. In Table 2.2, we compare the values of density obtained from different correlations. Vargaftik et al. [1996], Span and Wagner [1996], Angus et al. [1976], and Altunin [1975] provide similar results. The densities in columns E and F are calculated values using the Redlich Kwong equation of state with Morris and Turek [1986] and Spycher et al. [2003] parameters respectively. Parameters for the Morris and Turek [1986] correlation were fitted to match actual experimental density data values, whereas those for the Spycher et al. [2003] correlation were optimized to obtain the best fugacity coefficients that fit actual mutual solubility data in the 
(a)

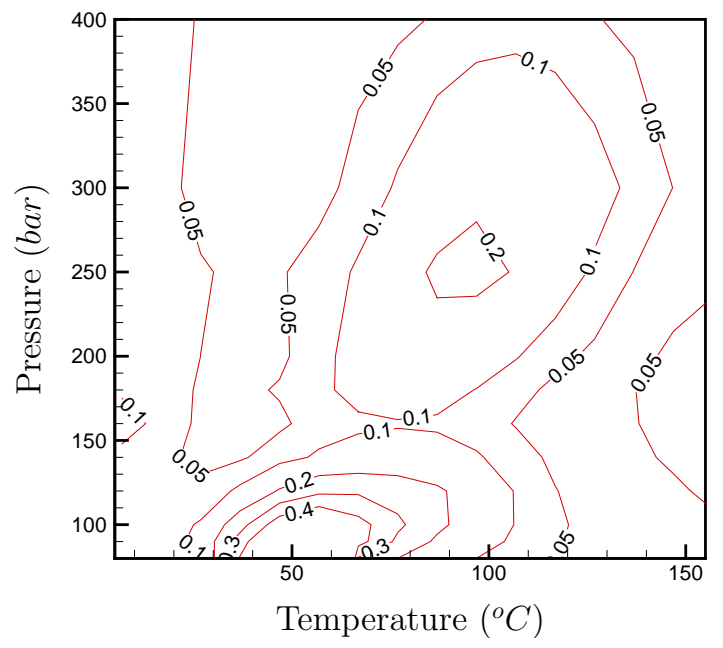

(b)

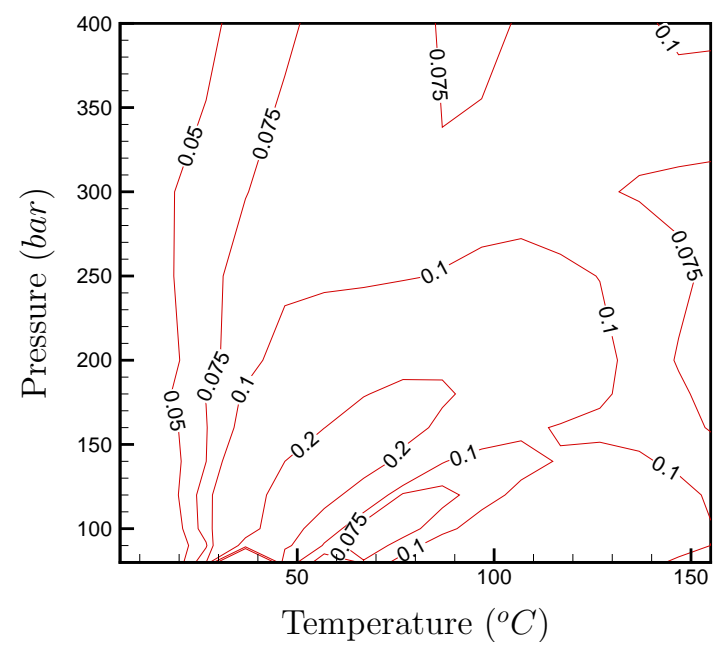

Figure 2.4: Relative deviation in percentage for the density of $\mathrm{CO}_{2}$, (a) Vargaftik et al. [1996] vs. Altunin [1975] and (b) Span and Wagner [1996] vs. Altunin [1975]

system $\mathrm{CO}_{2}-\mathrm{H}_{2} \mathrm{O}$.

\subsubsection{Enthalpy}

The calorific properties including enthalpy of $\mathrm{CO}_{2}$ have been less studied experimentally than thermal properties. The list of experimental studies in which direct or indirect measurements of $\mathrm{CO}_{2}$ enthalpy have been made is comparatively short. Furthermore, the accuracy of the experimental data is not high in the majority of studies, so that compilers of tables of thermodynamic properties hardly ever use the results of measured $\mathrm{H}$, but calculate the enthalpy from PVT data. Values for thermal properties such as internal energy, enthalpy, and heat capacities can be obtained from the equations of state presented in the previous section. The starting point for the derivation is the first law of thermodynamics. For a homogeneous closed system of $n$ moles, the first law of thermodynamics can be written as:

$$
d(n U)=\delta Q+\delta W
$$


Table 2.2: Carbon dioxide density $\left(\mathrm{kg} / \mathrm{m}^{3}\right)$ at $320 \mathrm{~K}$

\begin{tabular}{ccccccc}
\hline$P$ [bar $]$ & $\mathrm{A}$ & $\mathrm{B}$ & $\mathrm{C}$ & $\mathrm{D}$ & $\mathrm{E}$ & $\mathrm{F}$ \\
\hline 80 & 233.86 & 231.33 & 232.21 & 232.21 & 223.64 & 234.10 \\
100 & 447.03 & 446.78 & 449.77 & 449.77 & 465.68 & 505.36 \\
120 & 632.51 & 632.03 & 633.86 & 633.86 & 622.76 & 624.86 \\
160 & 747.38 & 746.09 & 747.32 & 747.32 & 734.66 & 725.30 \\
200 & 803.21 & 802.24 & 803.11 & 803.24 & 798.04 & 784.54 \\
300 & 883.39 & 883.01 & 883.73 & 883.78 & 895.91 & 877.51 \\
\hline
\end{tabular}

A: Table values from Vargaftik et al. [1996]

B: Calculated values from Span and Wagner [1996]

C: Table values from Angus et al. [1976]

D: Calculated values from Altunin [1975]

E: Redlich Kwong with Morris and Turek [1986] parameters

F: Redlich Kwong with Spycher et al. [2003] parameters

where $U$ is the internal energy of the system and $\delta Q$ and $\delta W$ are the small quantity of heat given to the system and small quantity of work done by the system, respectively. For reversible closed systems processes the differential work and heat can be written as:

$$
\begin{gathered}
\delta W=-P d(n V) \\
\delta Q=T d(n S)
\end{gathered}
$$

where $S$ and $V$ are the molar values of entropy and volume. Together these three 
equations give:

$$
d(n U)=T d(n S)-P d(n V)
$$

The appearance of the group $U+P V$ hints the definition of a new term called enthalpy (Dutch physicist H. Kamerlingh Onnes, 1908).

$$
H \equiv U+P V
$$

Two additional properties, also defined for convenience, are the Helmholtz energy,

$$
A \equiv U-T S
$$

and the Gibbs energy

$$
G \equiv H-T S
$$

Each one of these new defined properties leads to an equation similar to Equation 2.11

$$
\begin{aligned}
& d(n H)=T d(n S)+(n V) d P \\
& d(n A)=-P d(n V)-(n S) d T \\
& d(n G)=(n V) d P-(n S) d T
\end{aligned}
$$

These relations, with the application of Maxwell's equations [Prausnitz et al., 1986], can be used to expressed the enthalpy as a function of $\mathrm{T}$ and $\mathrm{P}$. Consider 
the first temperature derivative of enthalpy (Equation 2.15) at constant $T$ (also the definition of heat capacity $C_{p}$ ).

$$
\left(\frac{\partial H}{\partial T}\right)_{P}=T\left(\frac{\partial S}{\partial T}\right)_{P}=C_{p}
$$

Pressure derivative at constant temperature for entropy and enthalpy are

$$
\left(\frac{\partial S}{\partial P}\right)_{T}=-\left(\frac{\partial V}{\partial T}\right)_{P}
$$

and

$$
\left(\frac{\partial H}{\partial P}\right)_{T}=T\left(\frac{\partial S}{\partial T}\right)_{T}+V=V-T\left(\frac{\partial V}{\partial T}\right)_{p}
$$

The functional relation we are seeking is $H=H(T, P)$, whence

$$
d H=\left(\frac{\partial H}{\partial T}\right)_{P} d T+\left(\frac{\partial H}{\partial P}\right)_{T} d P=C_{p} d T+\left[V-T\left(\frac{\partial V}{\partial T}\right)_{P}\right] d P
$$

provides the equation relating the enthalpy of the fluids to temperature and pressure. At constant temperature Equation 2.21 and 2.19 become:

$$
\begin{gathered}
d H=\left[V-T\left(\frac{\partial V}{\partial T}\right)_{P}\right] d P \\
d S=-\left(\frac{\partial V}{\partial T}\right)_{P} d P
\end{gathered}
$$

An expression for the enthalpy can therefore be obtained after integration

$$
H=\int_{0}^{P}\left[V-T\left(\frac{\partial V}{\partial T}\right)_{P}\right] d P+H^{0}
$$

where $H^{0}$ is the enthalpy of pure $\mathrm{CO}_{2}$ as an ideal gas at temperature $T$. Equation 
2.24 provides a direct expression for enthalpy with independent variables $P$ and $T$. Alternatively, an expression with independent variables $V$ and $T$ can be obtained as:

$$
H=\int_{V}^{\infty}\left[P-T\left(\frac{\partial P}{\partial T}\right)_{V}\right] d V+P V+U^{0}
$$

where $U^{0}$ is the molar energy of pure $\mathrm{CO}_{2}$ as an ideal gas at temperature $T$.

\subsubsection{Enthalpies from PVT equations of state}

This section explains the application of PVT equations of state to the calculation of enthalpies. Let $H$ be the value of the enthalpy of a pure $\mathrm{CO}_{2}$ at some $P, T$. If $H^{0}$ is defined to be the value of $H$ at the same temperature but in an ideal-gas state and at a reference pressure $P^{0}$, then the enthalpy departure function is defined as $H-H^{0}$. As shown above, departure functions can be expressed in terms of PVT relations. In order to obtain the form of the departure function of enthalpy we first find an expression for the departure function for the Helmholtz energy. The variation in the Helmholtz energy with molar volume $V$ is given by:

$$
d A=-P d V
$$

Integrating at constant temperature from the reference volume $V^{0}$ to the system volume $V$ gives:

$$
A-A^{0}=\int_{V^{0}}^{V} P d V
$$

We break the integral into two parts:

$$
A-A^{0}=\int_{\infty}^{V} P d V-\int_{V^{0}}^{\infty} P d V
$$


The first integral requires real-gas properties, that is, $P=f(V)$ at constant temperature $T$, whereas the second is written for an ideal gas. To avoid the difficulty introduced by the infinity limit, we add and subtract $\int_{\infty}^{V}(R T / V) d V$ from the righthand side. Then

$$
A-A^{0}=\int_{\infty}^{V}\left(P-\frac{R T}{V}\right) d V-R T \ln \frac{V}{V^{0}}
$$

Other departure functions are readily obtained:

$$
S-S^{0}=\frac{-\partial}{\partial T}\left(A-A^{0}\right)_{V}=\int_{\infty}^{V}\left[\left(\frac{\partial P}{\partial T}\right)_{V}-\frac{R}{V}\right] d V+R \ln \frac{V}{V^{0}}
$$

and finally:

$$
H-H^{0}=\left(A-A^{0}\right)+T\left(S-S^{0}\right)+R T(Z-1)
$$

Estimation of enthalpy can easily be obtained using cubic EOS, as the integrals and derivatives in Equations 2.29 and 2.30 are relatively easy to evaluate analytically for the most common cubic PVT relations [Reid et al., 1987]. These equations are also used for more complex correlations, such as the ones by Altunin [1975] and Span and Wagner [1996]. Altunin's correlation uses the equations derived above to compute the enthalpy of pure $\mathrm{CO}_{2}$ from a non linear correlation for $Z$ given by:

$$
Z=1+\rho_{r} \sum_{i=0}^{9} \sum_{j=0}^{J_{i}} b_{i j}\left(T_{r}-1\right)^{j}\left(\rho_{r}-1\right)^{i}
$$

Where $Z=P V / R T, \rho_{r}=\rho / \rho_{c}$ and $T_{r}=T_{c} / T$. The $b_{i j}$ in Equation 2.32 are the correlation coefficients. Details of the analytical derivatives of this equations are given in Angus et al. [1976] and Altunin [1975]. Figure 2.5 shows the contours of $\mathrm{CO}_{2}$ enthalpy as calculated using the correlations of Altunin [1975]. We compare these values with those reported by Vargaftik et al. [1996] and found minor deviations, less than $0.2 \%$ (Figure 2.6). 


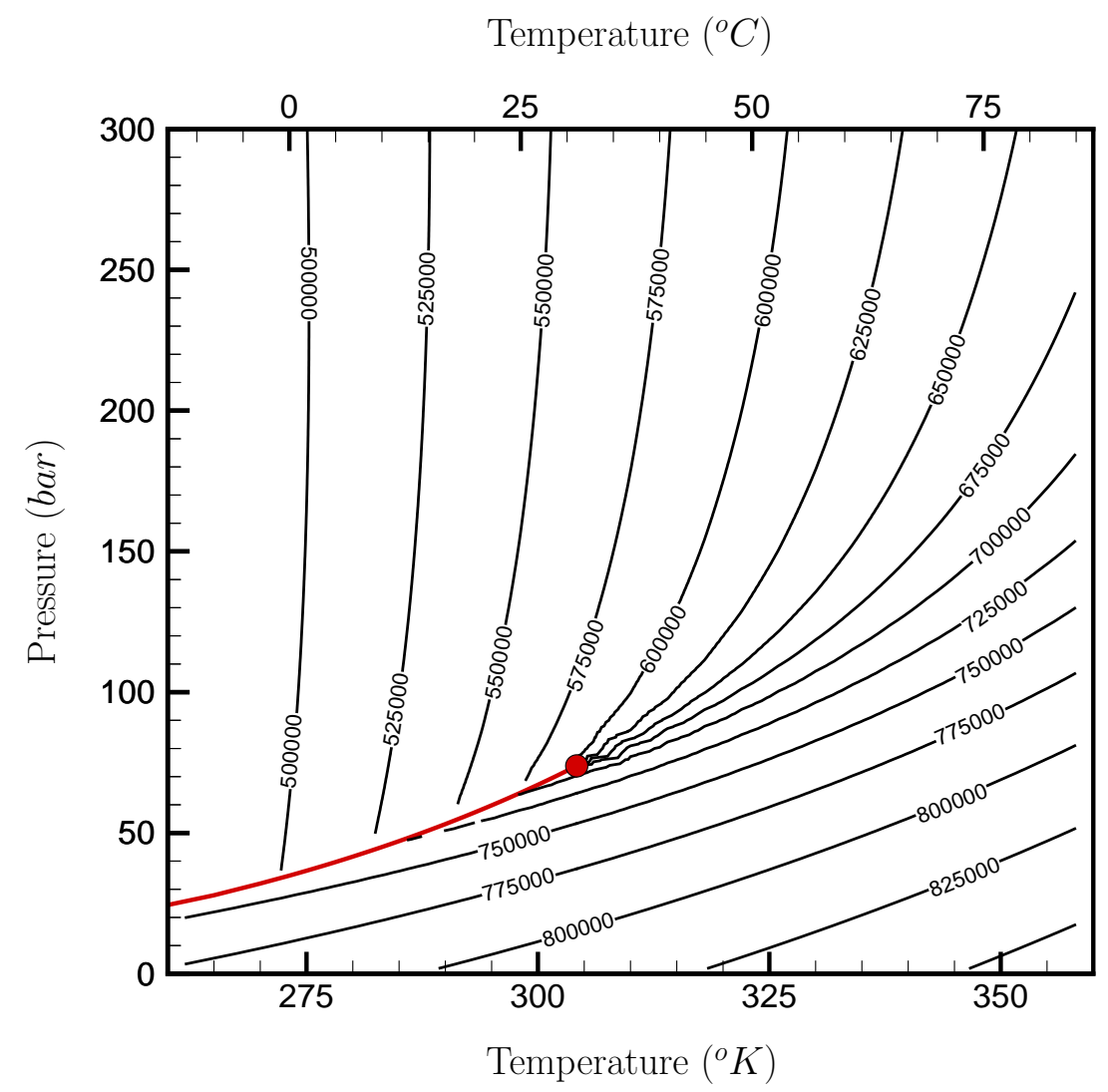

Figure 2.5: Calculated $\mathrm{CO}_{2}$ enthalpy $(\mathrm{J} / \mathrm{kg})$ using the correlations of Altunin [1975]

\subsubsection{Viscosity}

Altunin [1975] presented the first critical review of the viscosity of carbon dioxide, including experimental data from the early 1890's until the date of the publication of his monograph in 1975. Since then, several other correlations have been published including more recent and accurate data. However, according to a recent review by Vesovic et al. [1990], large discrepancies appear for the liquid-phase viscosity when comparing new and old sources of information. In an effort to reconcile the differences, they proposed a new correlation for the dynamic viscosity of $\mathrm{CO}_{2}$ which was later reviewed and updated by Fenghour et al. [1998]. Accurate representation of the viscosity of $\mathrm{CO}_{2}$ can be achieved if it is decomposed into three individual contribu- 


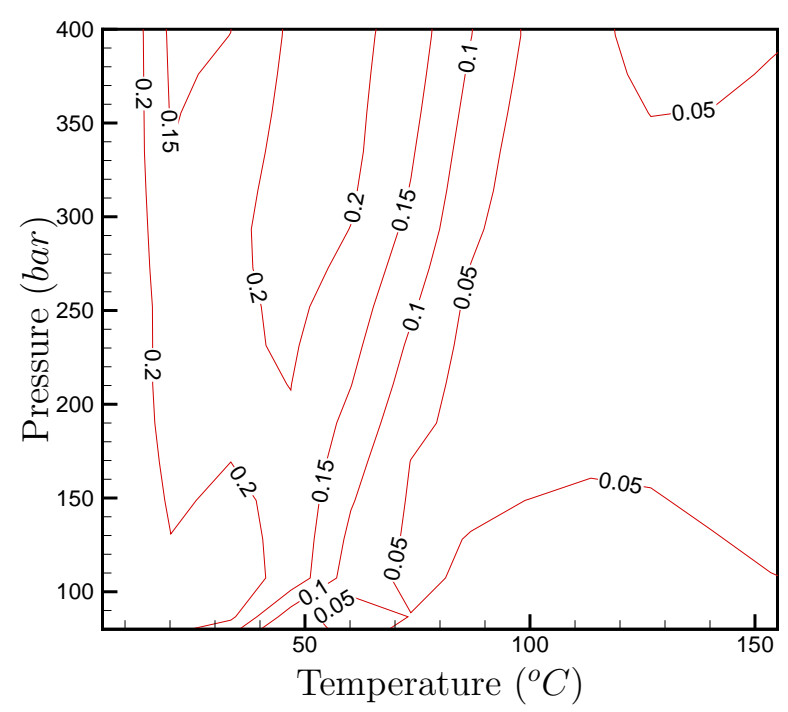

Figure 2.6: Relative deviation in percentage for the enthalpy of $\mathrm{CO}_{2}$ Vargaftik et al. [1996] vs. Altunin [1975]

tions:

$$
\eta(\rho, T)=\eta_{o}(T)+\Delta \eta(\rho, T)+\Delta \eta_{c}(\rho, T)
$$

or

$$
\eta(\rho, T)=\bar{\eta}(\rho, T)+\Delta \eta_{c}(\rho, T)
$$

where $\eta_{o}(T)$ is the viscosity in the zero-density limit, $\Delta \eta(\rho, T)$ an excess viscosity which represents the increase in the viscosity at elevated density over the dilute gas value at the same temperature, and $\Delta \eta_{c}(\rho, T)$ a critical enhancement accounting for the increase in the viscosity in the immediate vicinity of the critical point. The first two terms on the right hand side of Equation 2.33 are often regrouped and the resulting quantity is termed the background contribution $\bar{\eta}(\rho, T)$. Fenghour et al. [1998] provide a polynomial approximation for each one of the terms in Equation 2.33, 
(of degree 4 for $T$, degree 8 for $\rho$ ). A similar approach was followed by Altunin [1975]. Figure 2.7 shows the viscosity contours for $\mathrm{CO}_{2}$. Under supercritical conditions the viscosity of carbon dioxide is considerably lower than the water viscosity.

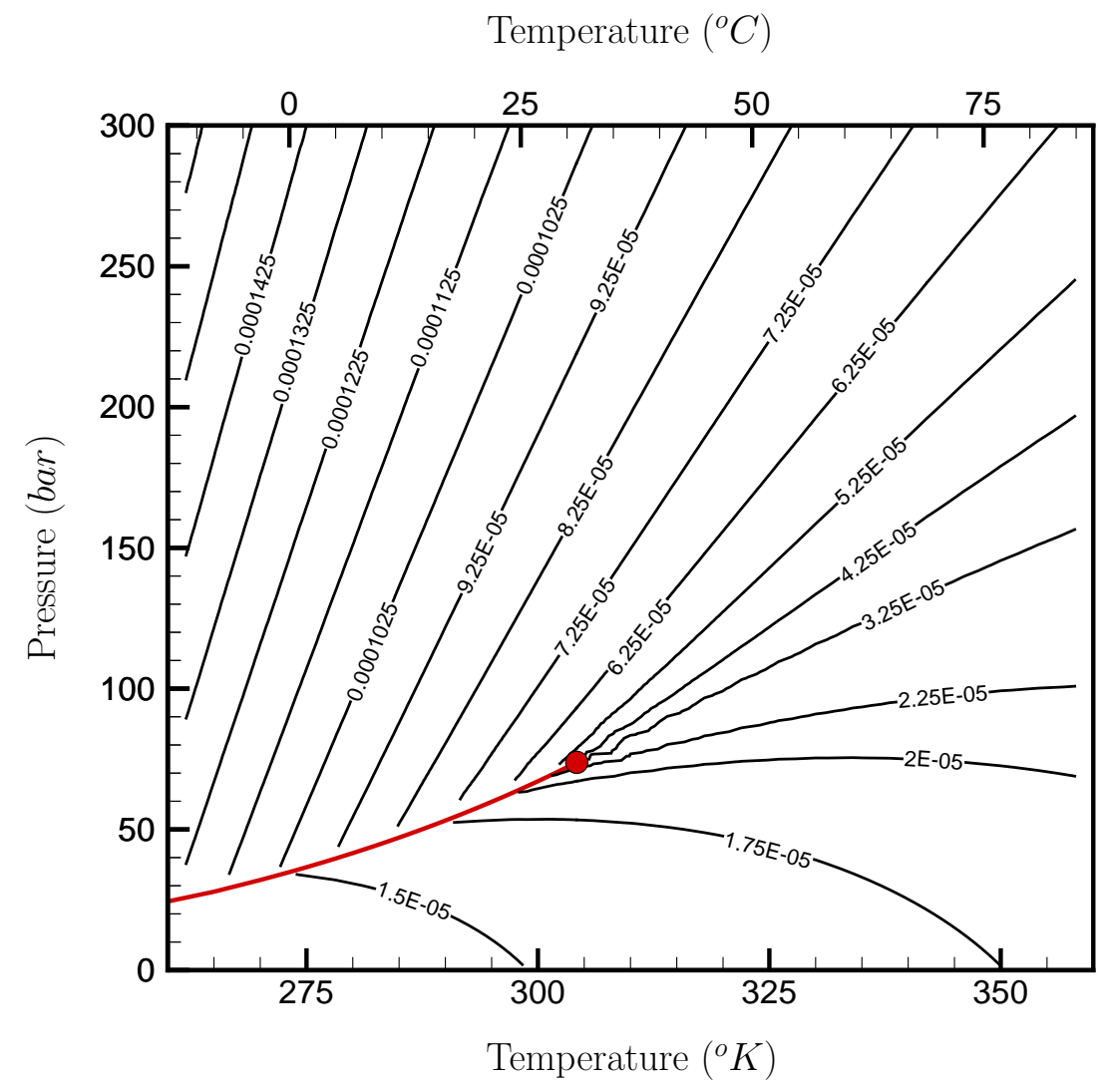

Figure 2.7: Calculated $\mathrm{CO}_{2}$ viscosity (Pa.s) using the correlations of Altunin [1975]

In order to assess the accuracy of Altunin's correlation for viscosity, we compare calculated values against data compiled by Vargaftik et al. [1996] (From data originally published by the State Bureau of Standard and Reference Data of USSR (GSSSD) GSSSD [1987]) and Fenghour et al. [1998]. Figure 2.8 shows that the predictions of Altunin's correlation for viscosity are reliable. In most of the T-P range of interest the error is below 2\%. For pressure less than 130 bar and temperatures between $40^{\circ} \mathrm{C}$ and $50^{\circ} \mathrm{C}$ the error can grow as much as $10 \%$, when comparing against the correlation of Vargaftik et al. [1996]. Deviations between Altunin [1975] and 
Fenghour et al. [1998] appear to be linked to density differences.

(a)

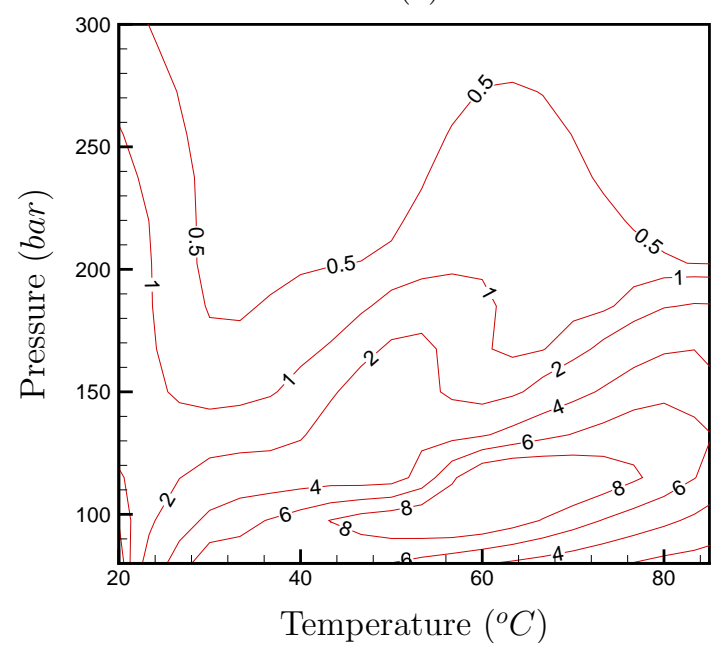

(b)

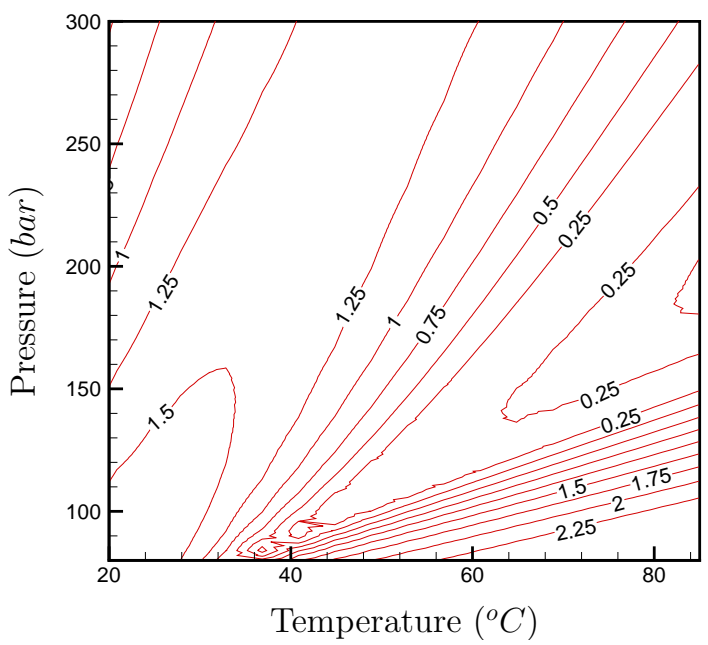

Figure 2.8: Relative deviation in percentage for the viscosity of $\mathrm{CO}_{2}$, (a) Vargaftik et al. [1996] vs. Altunin [1975] and (b) Fenghour et al. [1998] vs. Altunin [1975]

\subsection{Thermophysical properties of pure $\mathrm{H}_{2} \mathrm{O}$}

Thermophysical properties of pure $\mathrm{H}_{2} \mathrm{O}$ are well known. Since the introduction of the steam tables in 1967 by the International Formulation Committee (IFC) of the Sixth International Conference on the Properties of Steam, they became the standard reference for properties of water and steam. Volumetric properties of water are represented, within experimental error, by steam table equations as given by the IFC. Dynamic viscosity of water calculated from IFC's correlation reproduces experimental data within 2.5\%[IFC, 1967]. Critical and triple point data for $\mathrm{H}_{2} \mathrm{O}$ are presented in Table 2.3. 
Table 2.3: Triple and critical point parameters for $\mathrm{H}_{2} \mathrm{O}^{\mathrm{a}}$

\begin{tabular}{lcc}
\hline & \multicolumn{2}{c}{ Water $-\mathrm{H}_{2} \mathrm{O}$} \\
& Triple Point & Critical Point \\
\hline $\mathrm{P}($ bar $)$ & 0.006108 & 221.2 \\
$\mathrm{~T}\left({ }^{\circ} \mathrm{C}\right)$ & 0.0 & 374.12 \\
$\rho_{v}\left(\mathrm{~kg} / \mathrm{m}^{3}\right)$ & $4.8468 \times 10^{-3}$ & 317.8 \\
$\rho_{l}\left(\mathrm{~kg} / \mathrm{m}^{3}\right)$ & 999.8 & 317.8 \\
\hline \multicolumn{3}{c}{${ }^{\mathrm{a}}$ values reported by Vargaftik et al. $[1996]$}
\end{tabular}

\subsection{The system carbon dioxide-water}

In this section we present a description of the system carbon dioxide-water. The main objectives are to acquire a better understanding of the phase behavior and to provide simple but realistic models for predicting mutual solubilities of $\mathrm{CO}_{2} / \mathrm{H}_{2} \mathrm{O}$ mixtures.

\subsubsection{Phase behavior in the system carbon dioxide-water}

The binary system $\mathrm{H}_{2} \mathrm{O}-\mathrm{CO}_{2}$ is one of the fundamental systems in geochemical environments. It has been extensively studied, primary at high temperatures and pressures for applications in geothermal, metamorphism and ore deposit problems. The overall phase behavior and coexistence of different phases can be explored using the phase rule (Equation 2.1). For two components or species (carbon dioxide and water, $N=2$ ), the phase rule becomes $F=4-r$. Since there must be at least one phase $(r=1)$, the maximum number of phase-rule variables which must be specified to fix the state of the system is three: namely, $P, T$, and one mole (or mass) fraction. All equilibrium states of the system can therefore be represented in three-dimensional 
Pressure-Temperature-composition space (PTx). Early studies of the phase behavior of $\mathrm{CO}_{2}-\mathrm{H}_{2} \mathrm{O}$ by Tödheide and Franck [1963] depicted the PTx diagram for carbon dioxide-water equilibrium shown in Figure 2.9.

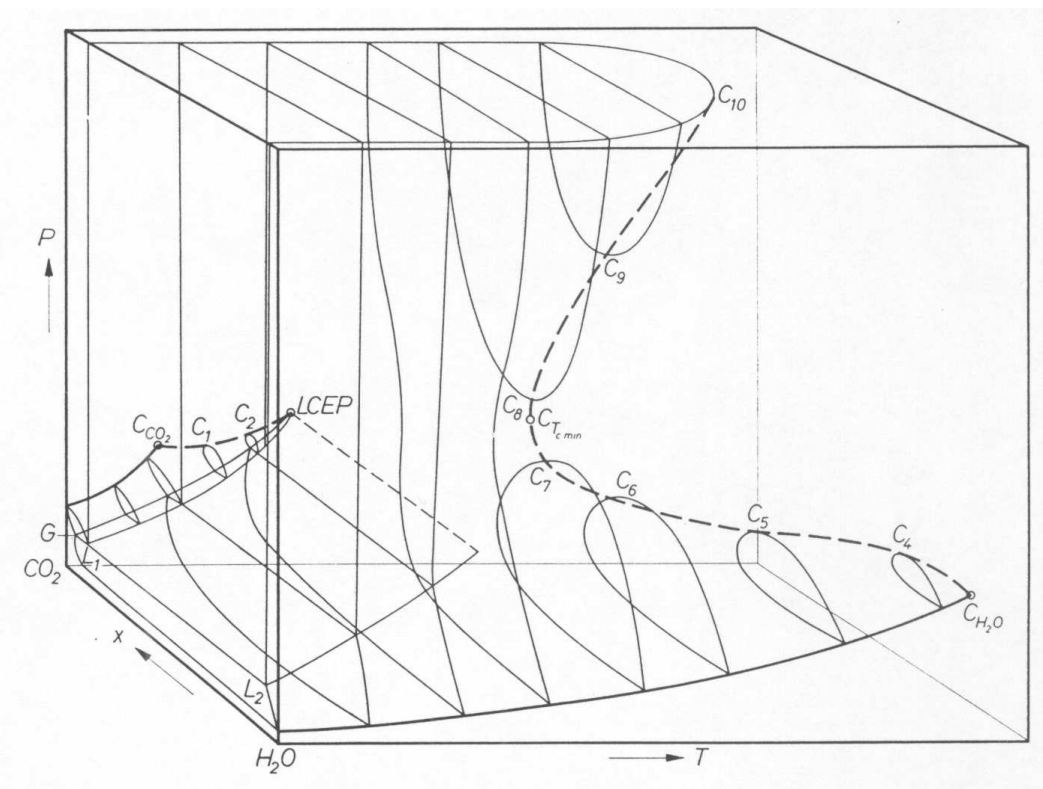

Figure 2.9: Pressure-temperature-composition diagram for the $\mathrm{CO}_{2}-\mathrm{H}_{2} \mathrm{O}$ system (from Tödheide and Franck [1963]).

The three-dimensional phase diagram shows schematically the P-T-composition surfaces which contain the equilibrium states between different phases. One critical line extends from the critical point of $\mathrm{CO}_{2}\left(\mathrm{C}_{\mathrm{CO}_{2}}\right)$ to the upper critical end point (UCEP) which is the termination of three-phase line $\mathrm{L}_{1} \mathrm{~L}_{2} \mathrm{~V}$ (vapor phase $\mathrm{V}$, water-rich liquid $\mathrm{L}_{1}, \mathrm{CO}_{2}$-rich liquid $\mathrm{L}_{2}$ ). The second critical line extends from the critical point of water $\left(\mathrm{C}_{\mathrm{H}_{2} \mathrm{O}}\right)$ until the melting curve at a pressure outside the range of experimental data [Evelein et al., 1976]. Because of the complexity of Figure 2.9, the detailed characteristics of the binary system are usually described by two-dimensional graphs that display what is seen on various planes that cut the three-dimensional diagram. One of these graphs is shown in Figure 2.10, as presented by Spycher et al. [2003]. This figure gives a more detailed view of the two- and three-phase coexistence curves 
at low temperatures on a PT cross section. From the data reported by Wendland et al. [1999] the upper critical end point for the mixture $\left(31.48{ }^{\circ} \mathrm{C}\right.$ and 74.11 bar $)$ and the critical point of $\mathrm{CO}_{2}\left(31.06{ }^{\circ} \mathrm{C}\right.$ and 73.85 bar $)$ are very close, making the coexistence lines $\mathrm{VL}$ and $\mathrm{L}_{1} \mathrm{~L}_{2} \mathrm{~V}$ almost coincide (Figure 2.10).

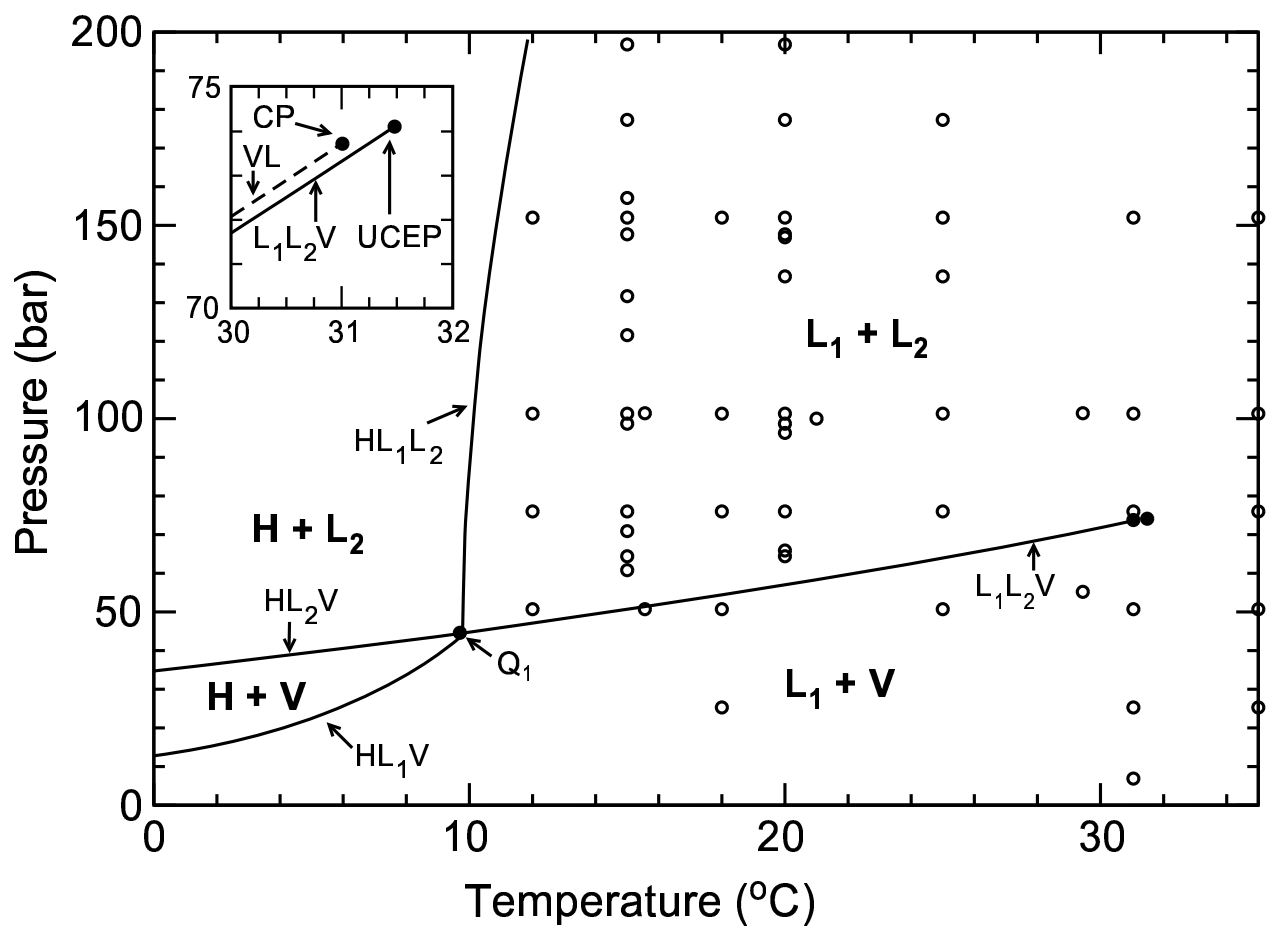

Figure 2.10: Enlarged P-T cross section of the $\mathrm{CO}_{2}-\mathrm{H}_{2} \mathrm{O}$ phase diagram, showing the two- and three-phase coexistence curves and the critical points, as presented by Spycher et al. [2003]. The circles are literature data points used by the authors in their mutual solubility model. The insert shows that the pure- $\mathrm{CO}_{2}$ liquid-vapor curve almost coincides with the three-phase coexistence curve for the $\mathrm{CO}_{2}-\mathrm{H}_{2} \mathrm{O}$ system. $\mathrm{Q}_{1}$ is the quadruple point for the system.

Figure 2.11 shows a Pressure-composition $(\mathrm{Px})$ cross section of the $\mathrm{CO}_{2}-\mathrm{H}_{2} \mathrm{O}$ phase diagram at $25^{\circ} \mathrm{C}$, as presented by Spycher et al. [2003]. It is important to note here that the region of three-phase coexistence is very narrow (see insert in Figure 2.11). Temperature-composition diagrams at 200 and 350 bar are shown in Figure 2.12 (from Bowers [1982]). From Figures 2.10-2.12 it can be concluded that for geologic sequestration, the three-phase region is of little importance and that the primary 
phases expected to be encountered are $\mathrm{H}_{2} \mathrm{O}$ - rich liquid and $\mathrm{CO}_{2}$-rich gas.

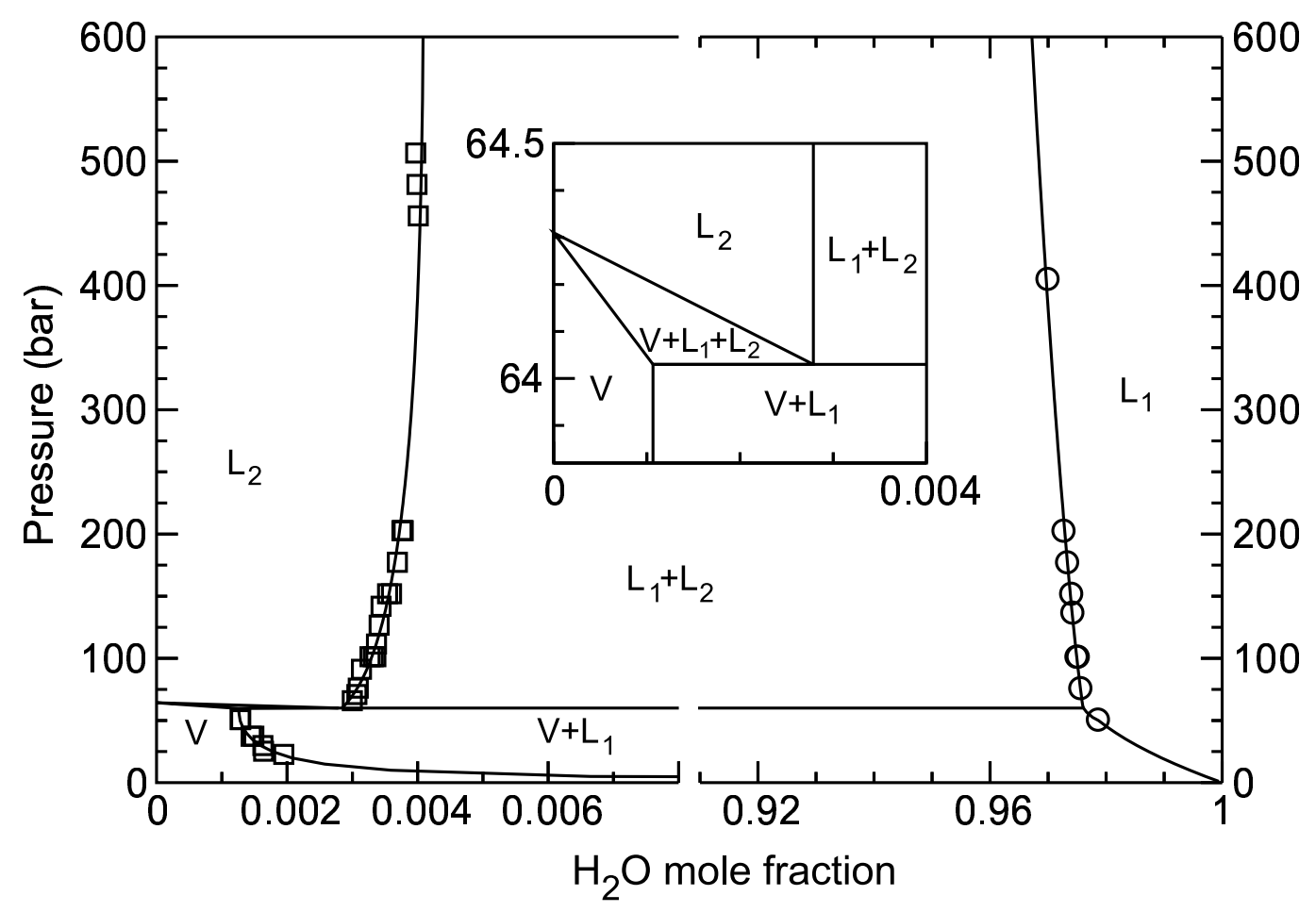

Figure 2.11: Pressure-composition cross section of the $\mathrm{CO}_{2}-\mathrm{H}_{2} \mathrm{O}$ phase diagram at 25 ${ }^{o} \mathrm{C}$. Circles represent data points for $\mathrm{CO}_{2}$ solubility in $\mathrm{H}_{2} \mathrm{O}$; squares are the for $\mathrm{H}_{2} \mathrm{O}$ solubility in $\mathrm{CO}_{2}$.

\subsubsection{Solubility models}

$\mathrm{CO}_{2}$ solubility data in pure water has been measured for a wide range of temperatures and pressures (Wiebe and Gaddy [1940, 1941]; Wiebe et al. [1933], Tödheide and Franck [1963], and Takenouchi and Kennedy [1964]). Spycher et al. [2003] and Duan and Sun [2003] provided extensive reviews of experimental solubility data for the system $\mathrm{CO}_{2}-\mathrm{H}_{2} \mathrm{O}$. The simplest models for vapor and liquid equilibrium are given by Raoult's and Henry's Law. Raoult's law assumes that the vapor is an ideal gas and the liquid phase is an ideal solution.

$$
y_{i} P=x_{i} P_{i}^{s a t}
$$



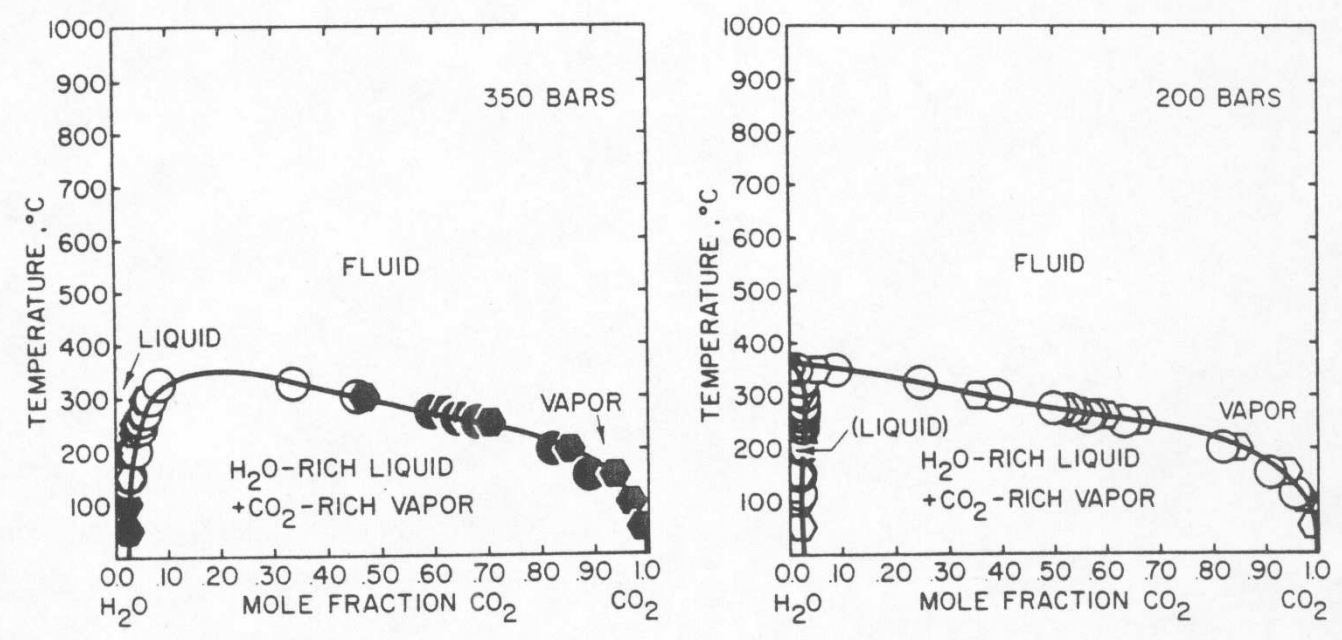

Figure 2.12: Temperature-composition diagram for the $\mathrm{CO}_{2}-\mathrm{H}_{2} \mathrm{O}$ system at 200 and 350 bars (from Bowers [1982]). Circles represent solubility data of Takenouchi and Kennedy [1964]; hexagons represent solubility data of Tödheide and Franck [1963].

where $y_{i}$ and $x_{i}$ are the mole fractions of component $i$ in the vapor and liquid phase respectively. Raoult's law requires $P_{i}^{\text {sat }}$ at temperature of application and is not appropriate for species whose critical temperature is less than the temperature of application. On the other hand, for volatile species present as a very dilute solute in the liquid phase, Henry's law states that the partial pressure of the species in the vapor phase is directly proportional to its liquid-phase mole fraction.

$$
y_{i} P=x_{i} K_{H_{i}}
$$

where $K_{H_{i}}$ is the Henry's constant. Experimental data on solubility of $\mathrm{CO}_{2}$ in $\mathrm{H}_{2} \mathrm{O}$ shows that the two previous model fail to accurately represent the equilibrium between phases (García [2001], Spycher et al. [2003]), therefore a more rigorous approach needs to be taken.

Consider the general case of a single-phase, open system that can interchange 
material with the surroundings. The total Gibbs energy $n G$ is still a function of $T$ and $P$, but since material can be taken or added, it is also function of the number of moles of chemical species present.

$$
n G=g\left(P, T, n_{1}, n_{2}, \ldots, n_{i}, \ldots\right)
$$

Using the definition of chemical potential:

$$
\mu_{i} \equiv\left[\frac{\partial(n G)}{\partial n_{i}}\right]_{P, T, n_{j}}
$$

the total differential of $n G$ is given by:

$$
d(n G)=(n V) d P-(n S) d T+\sum_{i} \mu_{i} d n_{i}
$$

Equation 2.39 is the fundamental property relation for systems with constant or variable mass and composition. If we consider two phases in equilibrium, Equation 2.39 can be rewritten for each phase with superscripts $\alpha$ and $\beta$ to identify the individual phases. The change in the total Gibbs energy would be the sum of these equations. Therefore, the two phases at the same temperature and pressure are in equilibrium when the chemical potential of each species is the same in all phases,

$$
\mu_{i}^{\alpha}=\mu_{i}^{\beta}
$$

Equation 2.40 is referred as the Gibbs criterion for equilibrium. The problem in the application of this criterion is that chemical potential cannot be measured directly. In order to overcome this inconvenience, Equation 2.39 can be used to express the chemical potential differential as:

$$
d \mu_{i}=-S_{i} d T+V_{i} d P
$$


For the case of an ideal gas at constant $\mathrm{T}$ we have

$$
\left(\frac{\partial \mu_{i}}{\partial P}\right)_{T}=V_{i}=\frac{R T}{P}
$$

which after integration becomes

$$
\mu_{i}-\mu_{i}^{0}=R T \ln \frac{P}{P^{0}}
$$

where the superscript ${ }^{0}$ signifies a reference state. Equation 2.43 relates the chemical potential to the experimental quantities $P$ and $T$. To extend this formulation to non-ideal systems, Lewis defined a function $f$ called the fugacity as

$$
\mu_{i}-\mu_{i}^{0}=R T \ln \frac{f_{i}}{f_{i}^{0}}
$$

For ideal gases the fugacity is equal to the pressure, and for mixtures of ideal gases, the fugacity is equal to the partial pressure. If one considers again two phases $\alpha$ and $\beta$, the Gibbs-Lewis equilibrium criterion is dictated by:

$$
f_{i}^{\alpha}=f_{i}^{\beta}
$$

Henry's law (Equation 2.36) assumes that the gas fugacity is equal to the partial pressure. This assumption is not necessary and is removed by including the gasphase fugacity coefficient $\Phi$ :

$$
f_{2}=\Phi_{2} y_{2} P=K_{H_{2,1}} x_{2}
$$

where 1 stands for solvent $\left(\mathrm{H}_{2} \mathrm{O}\right)$ and 2 stands for solute $\left(\mathrm{CO}_{2}\right)$. In general, we define 
the fugacity coefficient of component $i, \Phi_{i}$, as

$$
\Phi_{i}=\frac{f_{i}}{y_{i} P}
$$

Under ideal gas conditions the fugacity coefficient is equal to unity. The more the fugacity coefficient deviates from unity, the greater the system deviates from ideal gas behavior. Figure 2.13 shows the fugacity coefficient of $\mathrm{CO}_{2}$ calculated using the correlation of Spycher et al. [2003]. The figure demonstrates the excellent agreement between the values reported by Angus et al. [1976] and Spycher et al. [2003] correlation.

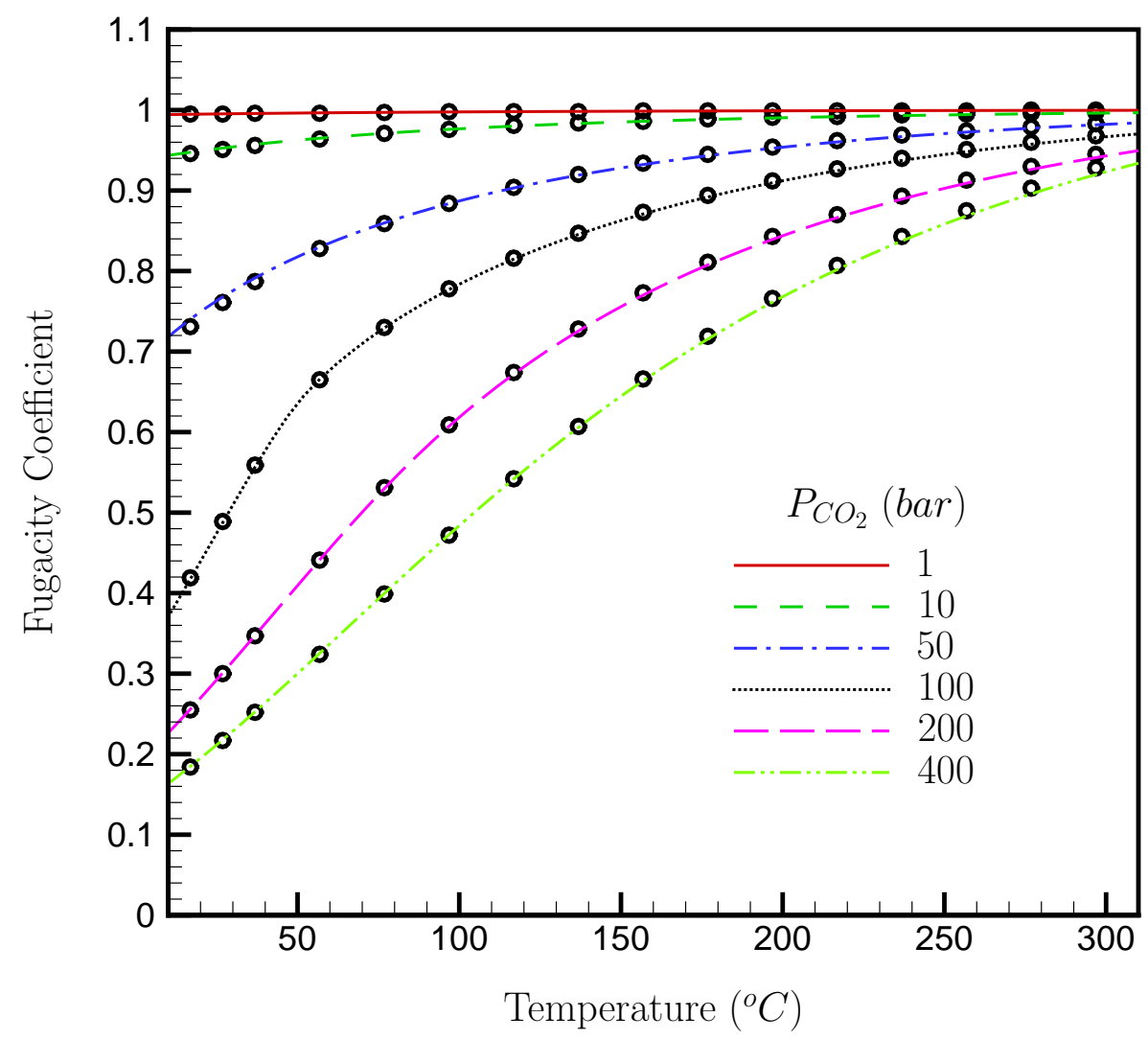

Figure 2.13: Fugacity coefficients for a range of temperature and pressure conditions. Lines are calculated from the correlation given by Spycher et al. [2003]; symbols represent the data of Angus et al. [1976]. 
At $\mathrm{CO}_{2}$ pressures in excess of 100 bar, a correction to Henry's coefficient to account for the pressure dependence of solute becomes important (Poynting correction). As it was used in Equation 2.46, Henry's coefficient is not a function of the composition but depends on temperature. The effect of pressure in the solubility of $\mathrm{CO}_{2}$ can be take into account by using the exact equation [Prausnitz et al., 1986]:

$$
\left(\frac{\partial \ln f_{2}^{L}}{\partial P}\right)_{T, x}=\frac{\bar{V}_{2}}{R T}
$$

where $\bar{V}_{2}$ is the partial molar volume of $\mathrm{CO}_{2}$ in the liquid phase. The thermodynamic definition of Henry's constant is:

$$
K_{H_{2,1}} \equiv \lim _{x_{2} \rightarrow 0} \frac{f_{i}^{L}}{x_{2}}
$$

Or, from l'Hôpital's rule

$$
K_{H_{2,1}} \equiv \lim _{x_{2} \rightarrow 0} \frac{d f_{i}^{L}}{d x_{2}}
$$

Substitution of Equation 2.50 into Equation 2.48 gives:

$$
\left(\frac{\partial \ln K_{H_{2,1}}}{\partial P}\right)_{T}=\frac{\bar{V}_{2}^{\infty}}{R T}
$$

where $\bar{V}_{2}^{\infty}$ is the partial molar volume of $\mathrm{CO}_{2}$ in the liquid phase at infinite dilution (i.e. $x_{2} \rightarrow 0$ ). After a final integration of Equation 2.51, we obtain a generalized form of Henry's law:

$$
\ln \frac{f_{2}}{x_{2}}=\ln K_{H_{2,1}}+\frac{\bar{V}_{2}^{\infty}\left(P-P_{1, s a t}\right)}{R T}
$$


Equation 2.52, also known as the Krichevsky-Kasarnovsky equation, can be used to calculate the mole fraction of $\mathrm{CO}_{2}$ in the aqueous phase $\left(x_{2}\right)$ with $P, P_{1, s a t}, K_{H_{2,1}}$, and $f_{2}$ as inputs. Without sacrificing accuracy, it is considered safe to assume that $y_{2}=1$ in the calculation of the gas fugacity, therefore $f_{2}=\Phi P$. For the calculation of Henry's constant we selected the correlation reported by Battistelli et al. [1997] that was computed using polynomial regression for data from 0 to $300^{\circ}$ published by Cramer [1982]. The correlation reported by Battistelli et al. [1997] was checked against the calculated values from Carroll et al. [1991], Scharlin [1996] and Drummond [1981] (see Figure 2.14). Figure 2.15 shows that the calculated values of $\mathrm{CO}_{2}$ mole fraction in the aqueous phase, using Equation 2.52 with a partial molar volume of $\bar{V}_{2}^{\infty}=32.1 \mathrm{~cm}^{3} /$ mole [Spycher et al., 2003], are within experimental uncertainty (about $7 \%$ ).

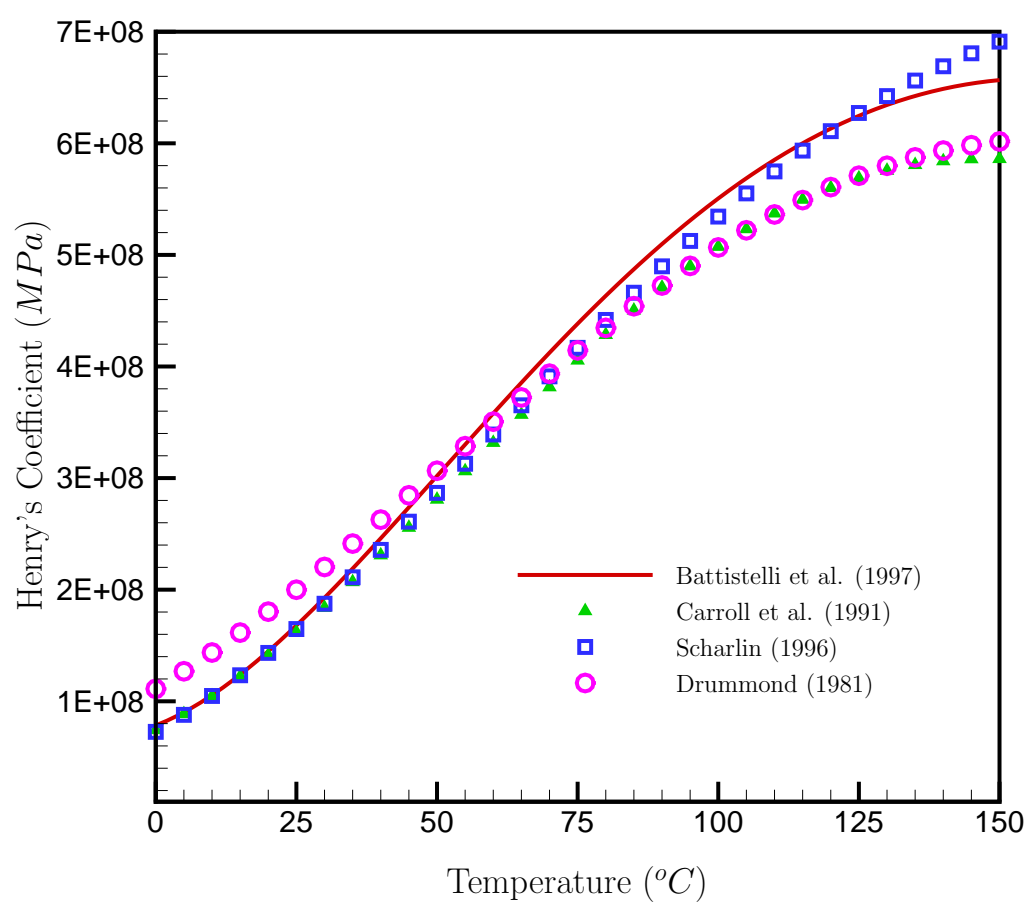

Figure 2.14: Temperature dependence of Henry's coefficient. 


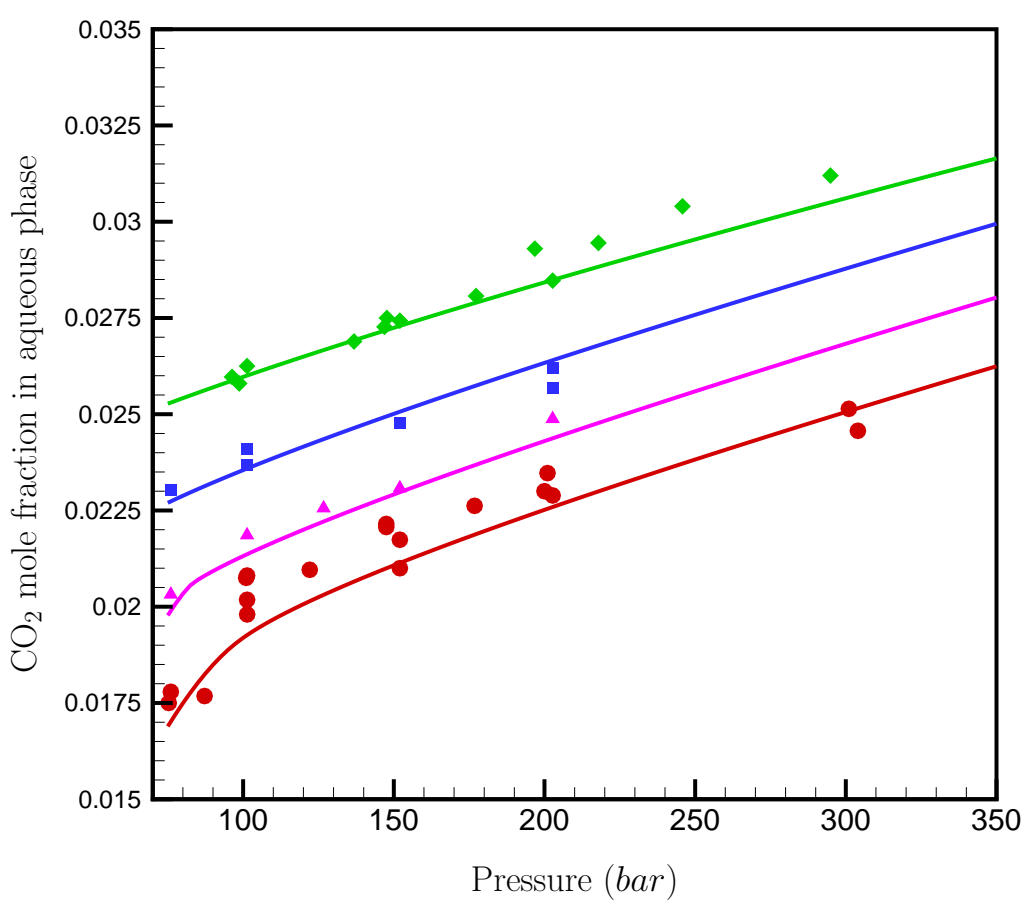

Figure 2.15: Solubility of carbon dioxide in pure water at 20, 31, 40, and $50{ }^{\circ} \mathrm{C}$. Lines are calculated from Equation 2.52; symbols represent experimental data compiled by Spycher et al. [2003].

Water partitioning into the $\mathrm{CO}_{2}$-rich vapor phase can be treated in similar fashion [Spycher et al., 2003]. At the moderate temperatures of interest here water mole fraction in the $\mathrm{CO}_{2}$-rich phase is small. For simplicity, water partitioning into the $\mathrm{CO}_{2}$-rich phase is here modeled as an evaporation process. Water is assumed to be present in the gas phase at its saturated vapor pressure, $P_{v}=P_{\text {sat }}(T)$. Density of water vapor is calculated from steam table equations as $\rho_{v}=\rho_{v}\left(T, P_{\text {sat }}\right)$ (see Section 2.3). $\mathrm{CO}_{2}$ density is obtained from the correlations of Altunin [1975] (see Section 2.2). Total density of the gas phase is:

$$
\rho_{g}=\rho_{v}+\rho_{C O_{2}}
$$


Gas phase composition is characterized by the mass fraction of water as:

$$
Y_{1}=\frac{\rho_{v}}{\rho_{C O_{2}}+\rho_{v}}
$$

This model underestimates the water content of the gas, though not by a large factor. A more accurate correlation has recently been developed by Spycher et al. [2003] .

\subsection{Thermophysical Properties of Fluid Mixtures}

Density, enthalpy and viscosity models for liquid and gas mixtures are described in this section. A large number of data exists on the solubility of $\mathrm{CO}_{2}$ in water and aqueous $\mathrm{NaCl}$ solutions. In spite of their significance, there is limited experimental data on densities, enthalpies or viscosities of aqueous $\mathrm{CO}_{2}-\mathrm{NaCl}$ systems. Usually, there are two sets of data, one for the system $\mathrm{H}_{2} \mathrm{O}-\mathrm{NaCl}$ and another for the system $\mathrm{CO}_{2}-\mathrm{H}_{2} \mathrm{O}$. The models we intend to use to describe the thermophysical properties of fluid mixtures are those presented by Battistelli et al. [1997]. Their thermophysical description has been successfully implemented into the simulator TOUGH2, and extensively used to model geothermal reservoirs with brines and $\mathrm{CO}_{2}$.

\subsubsection{Density of aqueous solutions of $\mathrm{CO}_{2}$}

Experimental data show that the density increase due to salinity can be up to almost $20 \%$, whereas carbon dioxide content produces an increase in aqueous phase density on the order of at most 2 to $3 \%$ [García, 2001]. Because the salinity effect is considerable stronger, the dependency on $\mathrm{CO}_{2}$ content is often ignored. This assumption is perfectly acceptable in geothermal applications. Nevertheless, it can conceal key processes in fluid flow dynamics of carbon sequestration. Following Battistelli et al. [1997] aqueous phase (brine) density can be calculated as function of temperature, pressure and salinity from a correlation given by Phillips et al. [1981]. 
Halite $(\mathrm{NaCl})$ solubility is calculated from an equation due to Potter and Brown [1977] quoted in Chou [1987], and salinity effects on vapor pressure are represented with a correlation due to Hass [1976]. The effect of $\mathrm{CO}_{2}$ is taken into account using a correlation for the partial molar volume of $\mathrm{CO}_{2}$ in the aqueous phase developed by García [2001]. The corresponding aqueous solution density is given by:

$$
\rho_{a q}=\rho_{b}+M_{2} \cdot c-c \cdot \rho_{b} \cdot \bar{V}
$$

where $\bar{V}$ is the apparent molar volume of dissolved $\mathrm{CO}_{2}$ (function of $T$ ); $M_{2}$ is the molecular weight of $\mathrm{CO}_{2} ; \rho_{b}$ is the brine density without dissolved $\mathrm{CO}_{2}$, and $c$ is the $\mathrm{CO}_{2}$ concentration expressed by the number of moles of solute in $1 \mathrm{~m}^{3}$ of solution.

The gas phase density is calculated considering an ideal mixture of steam and pure $\mathrm{CO}_{2}$ gas (see Equation 2.53).

\subsubsection{Enthalpy of fluid mixtures}

Enthalpy of liquid phase is calculated assuming an ideal solution of brine and liquid $\mathrm{CO}_{2}$.

$$
H_{L}=\left(1-X_{C_{2}}\right) H_{b}\left(P, T, X_{N a C l}\right)+X_{C_{2} H_{C O}}\left(P_{C O 2}, T\right)-H_{s o l}
$$

where $H_{\text {sol }}$ is the heat of dissolution. Brine enthalpy is computed using the correlation of Michaelides [1981] including the corrections presented by Gudmundsson and Thrainsson [1989]. 


\subsubsection{Viscosity of fluid mixtures}

The only source of data on viscosities of aqueous $\mathrm{NaCl}$ solutions containing $\mathrm{CO}_{2}$ at high pressures is provided by Kumagai and Yokoyama [1999]. At fixed temperature and pressure, their data show that the viscosity of the solution increases with an increase in $\mathrm{NaCl}$ and $\mathrm{CO}_{2}$ concentrations. They proposed the following equation for viscosity:

$\eta=(a+b T) M_{\mathrm{NaCl}}+(c+d T) M_{\mathrm{NaCl}}^{1 / 2}+(e+f T) M_{\mathrm{CO}_{2}}+(g+h T) M_{\mathrm{CO}_{2}}^{2}+i(P-0.1)+\eta_{\mathrm{H}_{2} \mathrm{O},(\mathrm{P}=0.1)}$

where $\eta$ is $m P a . s, T$ is in $K, P$ is in $M P a, M_{N a C l}, M_{C_{2}}$ are the molarities of $\mathrm{NaCl}$ and $\mathrm{CO}_{2}$ in $m o l ~ \mathrm{~kg}^{-1}$, respectively, and $\eta_{\mathrm{H}_{2} \mathrm{O},(P=0.1)}$ is the viscosity of $\mathrm{H}_{2} \mathrm{O}$ at $T$ and $P=0.1$ Mpa . $a$ through $i$ are adjustable parameters: $a=3.85971, b=$ $-1.32561 \times 10^{-2}, c=-5.37539, d=1.90621 \times 10^{-2}, e=8.79552, f=-3.17229 \times 10^{-2}$, $g=-7.22769, h=2.64498 \times 10^{-2}, i=1.69956 \times 10^{-3}$.

\subsection{Phase equilibria in $\mathrm{CO}_{2}-\mathrm{H}_{2} \mathrm{O}-\mathrm{NaCl}$ systems}

Attempts to predict the overall behavior of the system $\mathrm{CO}_{2}-\mathrm{H}_{2} \mathrm{O}-\mathrm{NaCl}$ by a single EOS have been very limited. Most studies are either in the low or high range of pressures and temperatures.(Bowers and Helgeson [1983], Rumpf et al. [1994] and, Duan et al. [1995]). The work by Drummond [1981] provides a first attempt to model $\mathrm{CO}_{2}$ solubilities in aqueous salt solutions. However, out of the 506 experimental data

reported by Drummond [1981] none is inside the common range of $20 \leq T \leq$ $60^{\circ} \mathrm{C}$ and $80 \leq P \leq 350$ bar. Duan and Sun [2003], based on a review of large number of experimental data, proposed a model calculating $\mathrm{CO}_{2}$ solubility in pure water and aqueous $\mathrm{NaCl}$ solutions from 273 to $533 \mathrm{~K}$ and from 0 to 2000 bar. The 
thermodynamic model they proposed is based on a computationally demanding EOS that makes application to large numerical simulations impractical. We decided to account for the effect of salinity on $\mathrm{CO}_{2}$ solubility in aqueous solutions of $\mathrm{NaCl}$ using the models presented in Section 2.4 with a Henry's coefficient that depends on temperature and $\mathrm{NaCl}$ content [Battistelli et al., 1997]. $\mathrm{CO}_{2}$ solubility values reported by Duan et al. [1995] and those obtained from the proposed correlation are listed in Table 2.4.

Table 2.4: Solubility of carbon dioxide in brines (mole fractions)

\begin{tabular}{lccccccccc}
\hline & \multicolumn{2}{c}{$1 \mathrm{~m}$ aqueous $\mathrm{NaCl}$ solutions } & \multicolumn{2}{c}{$2 \mathrm{~m}$ aqueous $\mathrm{NaCl}$ solutions } \\
& \multicolumn{2}{c}{$T=30^{\circ} \mathrm{C}$} & \multicolumn{2}{c}{$T=60^{\circ} \mathrm{C}$} & \multicolumn{2}{c}{$T=30^{\circ} C$} & \multicolumn{2}{c}{$T=60^{\circ} C$} \\
$\mathrm{P}$ (bar) & {$[1]$} & {$[2]$} & {$[1]$} & {$[2]$} & {$[1]$} & {$[2]$} & {$[1]$} & {$[2]$} \\
\hline 50 & 0.0157 & 0.0151 & 0.0099 & 0.0090 & 0.0128 & 0.0119 & 0.0082 & 0.0073 \\
100 & 0.0197 & 0.0188 & 0.0151 & 0.0139 & 0.0161 & 0.0148 & 0.0126 & 0.0112 \\
200 & 0.0216 & 0.0209 & 0.0181 & 0.0169 & 0.0176 & 0.0165 & 0.0150 & 0.0137 \\
300 & 0.0232 & 0.0227 & 0.0198 & 0.0189 & 0.0191 & 0.0179 & 0.0165 & 0.0153 \\
400 & 0.0248 & 0.0248 & $0.0326^{\mathrm{a}}$ & 0.0206 & 0.0205 & 0.0193 & 0.0178 & 0.0167 \\
\hline
\end{tabular}

${ }^{\text {a }}$ suspected typo in Duan and Sun [2003]

[1] Duan and Sun [2003]

[2] Equation 2.52

\subsection{Recommendations and concluding remarks}

An extensive data base exists for the thermodynamic properties of water and carbon dioxide. Thermodynamic data for the aqueous solutions of $\mathrm{NaCl}$ and $\mathrm{CO}_{2}$ is relatively sparse and more uncertain. Until recently, few experimental data were 
available for the ternary system $\mathrm{CO}_{2}-\mathrm{H}_{2} \mathrm{O}-\mathrm{NaCl}$ at temperatures and pressures of interest for geologic carbon sequestration. Therefore, the application of a single EOS that predicts both volumetric properties (pure fluids and its mixtures) and phase equilibria for the system $\mathrm{CO}_{2}-\mathrm{H}_{2} \mathrm{O}-\mathrm{NaCl}$ is limited by the following observations:

1. The development of the majority of such EOS was based primarily on two sets of data, one at low pressures and temperatures and other at high pressures and temperatures, respectively, and

2. In order the make the EOS reliable for the entire $\mathrm{CO}_{2}-\mathrm{H}_{2} \mathrm{O}-\mathrm{NaCl}$ system at large $\mathrm{P}-\mathrm{T}$ range, the mathematical formulation is computationally demanding making its application to reservoir scale simulations impractical.

As it was discussed in this chapter, the necessary accuracy for the overall thermophysical behavior of the system $\mathrm{CO}_{2}-\mathrm{H}_{2} \mathrm{O}-\mathrm{NaCl}$ can be obtained by properly linking a set of individual models.

The following are recommended for estimating thermophysical properties of pure fluids:

- Use the correlations of Altunin [1975] for density, enthalpy and viscosity of carbon dioxide.

- Use the correlations of IFC [1967] for thermophysical properties of pure water. The following are recommended for solubility calculations between the $\mathrm{H}_{2} \mathrm{O}$-rich liquid and the $\mathrm{CO}_{2}$-rich vapor:

- Use the Krichevsky-Kasarnovsky equation to compute $\mathrm{CO}_{2}$ solubility in aqueous phase.

- Consider temperature and salinity dependence of Henry's coefficient according to Battistelli et al. [1997]. 
- Estimate $\mathrm{H}_{2} \mathrm{O}$ in vapor phase assuming an evaporation process. This could be improved if a mutual solubility model between water and carbon dioxide is considered [Spycher et al., 2003].

The following are recommended for estimating thermodynamic properties of fluid mixtures:

- Assume ideal mixtures for the vapor phase for the calculation of densities, viscosities and enthalpies.

- Calculate liquid phase density according to García [2001].

- Assume ideal solution for the calculation of liquid phase enthalpy.

- Use the model of Kumagai and Yokoyama [1999] for liquid viscosity, pending availability of additional data to confirm its accuracy. Otherwise, changes in water viscosity from $\mathrm{CO}_{2}$ dissolution could be ignored. 



\section{Chapter 3}

\section{Mathematical model and implementation}

\section{$3.1 \quad$ Introduction}

The numerical simulator for carbon sequestration into saline aquifers, which will be referred to as TOUGH2/ECO2, is presented in this chapter. TOUGH2/ECO2 is an Equation Of State (EOS) module based on a general integral finite difference code known

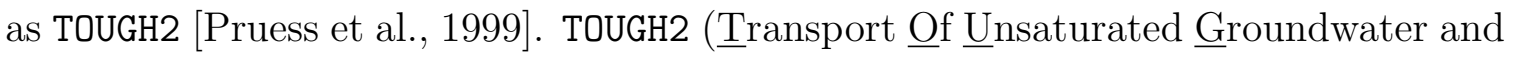
Heat) is a three-dimensional simulator for non-isothermal flows of multicomponent, multiphase fluids in porous and fractured media. Most of ECO2's features, capabilities, and functionality are inherited from TOUGH2's module EWASG [Battistelli et al., 1997], of which it is a descendant. The EWASG (WAter-Salt-Gas) fluid property module was developed for modeling geothermal reservoirs with saline fluids and non-condensible gas (NCG). EWASG addresses higher temperatures and lower $\mathrm{CO}_{2}$ partial pressures than would be encountered in aquifer disposal of $\mathrm{CO}_{2}$. The modeling capabilities for $\mathrm{CO}_{2}$ injection into saline aquifers presented in this chapter include realistic representation of thermophysical properties of sub- and supercritical $\mathrm{CO}_{2}$. Nevertheless, 
the present version of the simulator only handles supercritical pressures. The current implementation includes only two fluid phases (in addition to solid salt): an aqueous phase, and a single $\mathrm{CO}_{2}$-rich phase. A numerical methodology for simulating three fluid phases (Aqueous-Liquid-Gas) is currently under development (for additional details see Pruess [2003]).

Most of the material related to governing equations and numerical implementation presented in this chapter (Sections 3.2.1, 3.2.2, 3.2.3 and 3.3) follows closely the presentation of Pruess [1987] and Pruess et al. [1999] (TOUGH2 user's guide). A major part of the development of TOUGH2/ECO2 was carried out by Pruess and originally presented in Pruess and García [2002]. The ECO2 version discussed in this chapter includes additional enhancements in the phase equilibria model and thermophysical properties of fluid mixtures.

\subsection{Governing equations}

Here we present the mathematical model on which the simulator TOUGH2/ECO2 is based under the following assumptions:

1. Multiphase flow extension of Darcy's Law.

2. All the dependent variables are volumetric averages over a representative elementary volume (REV) [Bear, 1972].

3. No chemical reactions take place other than partitioning of mass components (species) among phases.

4. The phases are in local chemical and thermal equilibrium. The assumption of local chemical equilibrium presupposes that the component mass fraction in one phase determines the mass fraction in all other phases, and that component transport equations may be summed over all phases. 
5. Neglect effects of mechanical stress.

\subsubsection{Continuum balance equations}

The general balance equation for multiphase, non-isothermal, multicomponent system is

$$
\frac{\partial}{\partial t} \int_{V_{n}} M^{(\kappa)} d V_{n}=\int_{\Gamma_{n}} \mathbf{F}^{(\kappa)} \cdot \mathbf{n} d \Gamma_{n}+\int_{V_{n}} q^{(\kappa)} d V_{n}
$$

where $\kappa=1$ for water, $\kappa=2$ for salt, $\kappa=3$ for $\mathrm{CO}_{2}$, and $\kappa=4$ for heat. The integration is over an arbitrary subdomain $V_{n}$ of the flow system under study, which is bounded by the closed surface $\Gamma_{n}$. For $\kappa=1,2,3$, the quantity $M^{(\kappa)}$ is the mass

of component $\kappa$ per unit of reservoir volume, $\mathbf{F}^{(\kappa)}$ denotes the mass flux, and $q^{(\kappa)}$ denotes sinks and sources. For $\kappa=4, M^{(\kappa)}$ is the amount of energy (heat) per unit reservoir volume, $\mathbf{F}^{(\kappa)}$ is the heat flux, and $q^{(\kappa)}$ is the rate of heat generation per unit volume. $\mathbf{n}$ is a unit vector on the surface element $d \Gamma_{n}$, pointing inward into $V_{n}$.

\subsubsection{Accumulation terms}

The general form of the mass accumulation for water, salt and $\mathrm{CO}_{2}(\kappa=1,2,3)$ is

$$
M^{(\kappa)}=\phi \sum_{\beta} \rho_{\beta} S_{\beta} X_{\beta}^{(\kappa)}
$$

where the summation extends over all fluid phases $\beta(\beta=1,2,3$ indicates the gas $(\mathrm{G})$, liquid (L) and solid salt $(\mathrm{S})$ phases, respectively), because each of the mass components may partition and be present in more than one fluid phase. Other parameters appearing in Equation 3.2 are as follows: $\phi$ is the porosity, $\rho_{\beta}$ is the density of phase 
$\beta, S_{\beta}$ is the saturation of phase $\beta$ (i.e., the fraction of pore volume occupied by phase $\beta)$, and $X_{\beta}^{(\kappa)}$ is the mass fraction of component $\kappa$ in phase $\beta$. The heat accumulation term $(\kappa=4)$ includes contributions from both solid and fluid phases

$$
M^{(4)}=\phi \sum_{\beta} \rho_{\beta} S_{\beta} u_{\beta}+(1-\phi) \rho_{R} C_{R} T
$$

where $\rho_{R}$ and $C_{R}$ are density and specific heat of the rock grains, respectively, $T$ is the temperature, and $u_{\beta}$ is the specific internal energy of phase $\beta$.

\subsubsection{Flux terms}

A separate momentum balance equation is not typically included in balance equation formulations of multiphase systems. It is customary to substitute a multiphase form of Darcy's law into the flux terms in Equation 3.1 (advective mass flux). In addition, the mass flux $\mathbf{F}^{(\kappa)}$ may include a diffusive-dispersive component. The advective mass flux is

$$
\left.\mathbf{F}^{(\kappa)}\right|_{a d v}=\sum_{\beta} X_{\beta}^{(\kappa)} \mathbf{F}_{\beta}
$$

where the individual phase fluxes $\mathbf{F}_{\beta}$ are given by a multiphase extension of Darcy's law.

$$
\mathbf{F}_{\beta}=\rho_{\beta} \mathbf{u}_{\beta}=-\rho_{\alpha} \frac{\overline{\mathbf{k}} k_{r \beta}}{\mu_{\beta}}\left(\nabla P_{\beta}-\rho_{\beta} \mathbf{g}\right)
$$

where $\mathbf{u}_{\beta}$ is the Darcy velocity (volume flux) in phase $\beta, \overline{\mathbf{k}}$ is the intrinsic permeability tensor, $k_{r \beta}$ is the relative permeability of Phase $\beta$, and $\mathbf{g}$ is the vector of gravitational acceleration. 
The diffusive-dispersive flux is written [de Marsily, 1986]

$$
\left.\mathbf{F}^{(\kappa)}\right|_{d i s}=-\sum_{\beta} \rho_{\beta} \overline{\mathbf{D}}_{\beta}^{(\kappa)} \nabla X_{\beta}^{(\kappa)}
$$

where the hydrodynamic dispersion tensor is given by

$$
\overline{\mathbf{D}}_{\beta}^{(\kappa)}=D_{\beta, T}^{(\kappa)} \overline{\mathbf{I}}+\frac{\left(D_{\beta, L}^{(\kappa)}-D_{\beta, T}^{(\kappa)}\right)}{u_{\beta}^{2}} \mathbf{u}_{\beta} \mathbf{u}_{\beta}
$$

Here $\overline{\mathbf{I}}$ is the unit tensor, and $\mathbf{u}_{\beta} \mathbf{u}_{\beta}$ operates on a vector to the right in the sense of dot product, i.e, it contributes a term $\left.\mathbf{u}_{\beta}\left(\mathbf{u}_{\beta} \cdot \nabla X_{\beta}^{(\kappa}\right)\right)$ in Equation 3.6. The longitudinal and transverse dispersion coefficients are

$$
D_{\beta, L}^{(\kappa)}=\phi \tau_{0, \beta} d_{\beta}^{(\kappa)}+\alpha_{\beta, L} u_{\beta}
$$

and

$$
D_{\beta, T}^{(\kappa)}=\phi \tau_{0, \beta} d_{\beta}^{(\kappa)}+\alpha_{\beta, T} u_{\beta}
$$

respectively, where $d_{\beta}^{(\kappa)}$ is the molecular diffusion coefficient for component $\kappa$ in phase $\beta, \tau_{0, \beta}=\tau_{0} \tau_{\beta}$ is the tortuosity which includes a porous medium-dependent factor $\tau_{0}$ and a coefficient which depends on phase saturation $S_{\beta}, \tau_{\beta}=\tau_{\beta}\left(S_{\beta}\right)$, and $\alpha_{L}$ and $\alpha_{T}$ are the longitudinal and transverse dispersivities, respectively.

The heat flux includes both conduction and convection

$$
\mathbf{F}^{(4)}=-K \nabla T+\sum_{\beta} h_{\beta} \mathbf{F}_{\beta}
$$

where $K$ is the overall porous media thermal conductivity, $h_{\beta}$ is the specific enthalpy of phase $\beta$. 
Considering that the solubility of $\mathrm{NaCl}$ in the gas phase has been neglected and that solid salt phase is not mobile, the accumulation terms (Equation 3.2) and mass flux terms (Equation 3.4) for $\mathrm{NaCl}$ component $(\kappa=2)$ are written as follows:

$$
\begin{gathered}
M^{(2)}=\phi S_{S} \rho_{S}+\phi S_{L} \rho_{L} X_{L}^{(2)} \\
\mathbf{F}^{(2)}=-k \frac{k_{r L}}{\mu_{L}} \rho_{L} X_{L}^{(2)}\left(\nabla P_{L}-\rho_{L} \mathbf{g}\right)
\end{gathered}
$$

where $S_{S}$ is the "solid saturation", defined as the fraction of pore volume occupied by solid salt.

\subsubsection{Closure relations}

Closure of the system of balance equations requires a sufficient number of equations such that all unknowns can be determined. Closure can be accomplished by providing equations of state (EOS) and constitutive relations. For a non-isothermal system in which there are 3 components (water, salt, carbon dioxide), there are 4 equations (one equation per component plus one energy equation). However, there are many more dependent variables. After considering several simplifying assumptions regarding the $\mathrm{NaCl}$ component, a list of dependent variables is presented in Table 3.1. For example, the assumption that the solid phase (solid salt) is immobile $\left(\mathbf{u}_{S}=0\right)$ eliminates three unknowns $\left(k_{r S}, P_{S}, \mu_{S}\right)$ and the need to specify a constitutive relation to express its motion.

Independent variables total 28, therefore, to close the system of equations an additional 24 independent relationships are required. Equations to close the system are: 
Table 3.1: List of all dependent variables

\begin{tabular}{cc}
\hline Variables & Number \\
\hline$S_{L}, S_{S}, S_{G}$ & 3 \\
$X_{L}^{1}, X_{G}^{1}, X_{L}^{2}, X_{L}^{3}, X_{G}^{3}$ & $5^{\mathrm{a}}$ \\
$k_{r L}, k_{r G}$ & $2^{\mathrm{b}}$ \\
$P_{L}, P_{G}$ & 2 \\
$\rho_{L}, \rho_{S}, \rho_{G}$ & 3 \\
$u_{L}, u_{S}, u_{G}$ & 3 \\
$H_{L}, H_{S}, H_{G}$ & 3 \\
$\mu_{L}, \mu_{G}$ & 2 \\
$\phi$ & 1 \\
$K$ & 1 \\
$\tau_{0, G}, \tau_{0, L}$ & 28 \\
$T$ & 1 \\
\hline TOTAL & 28 \\
\hline
\end{tabular}

a Assumptions for $\mathrm{NaCl}$ component and solid salt phase:

$$
\begin{aligned}
& X_{S}^{1}=0, X_{G}^{2}=0, X_{S}^{2}=1, \\
& X_{S}^{3}=0
\end{aligned}
$$

b Immobile solid phase $k_{r S}=0$

1. Phase saturations sum to unity [one relationship]

$$
S_{G}+S_{L}+S_{S}=1
$$

2. Mass fractions in each phase sum to unity [two relationships]

$$
X_{L}^{(1)}+X_{L}^{(2)}+X_{L}^{(3)}=X_{G}^{(1)}+X_{G}^{(3)}=1
$$


3. Relative permeability functions and capillary pressure are assumed to be function of phase saturations. This approach, although supported by an abundance of experimental data is still subject of debate [Juanes, 2003; Yortsos and Huang, 1986]. Hysteresis phenomena that may be of considerable importance will not be considered in this work. [three relationships]

$$
\begin{aligned}
k_{r G} & =k_{r G}\left(S_{G}, S_{L}\right) \\
k_{r L} & =k_{r L}\left(S_{G}, S_{L}\right) \\
P_{c_{G L}} & =P_{G}-P_{L}=P_{c_{G L}}\left(S_{L}\right)
\end{aligned}
$$

4. Equations of state (equilibrium relationship between pressure, volume, temperature, and composition) and correlations for $u, h$, and $\mu$ [eleven relationships].

$$
\begin{aligned}
& \rho_{G}=\rho_{G}\left(P, T, X_{G}^{(1)}, X_{G}^{(3)}\right) \\
& \rho_{L}=\rho_{L}\left(P, T, X_{L}^{(2)}, X_{L}^{(3)}\right) \\
& \rho_{S}=\rho_{S}(P, T) \\
& u_{G}=u_{G}\left(P, \rho_{G}, H_{G}\right) \\
& u_{L}=u_{L}\left(P, \rho_{L}, H_{L}\right) \\
& u_{S}=u_{S}\left(P, \rho_{S}, H_{S}\right) \\
& H_{G}=H_{G}\left(P, T, X_{G}^{(1)}, X_{G}^{(3)}\right) \\
& H_{L}=H_{L}\left(P, T, X_{L}^{(1)}, X_{L}^{(2)}, X_{L}^{(3)}\right) \\
& H_{S}=H_{S}(P, T) \\
& \mu_{G}=\mu_{G}\left(P, T, X_{G}^{(1)}, X_{G}^{(3)}\right) \\
& \mu_{L}=\mu_{L}\left(P, T, X_{L}^{(2)}\right)
\end{aligned}
$$

5. Solubility and phase equilibria models [three relationships].

$$
X_{L}^{(2)}=X_{L}^{(2)}(T)
$$




$$
\begin{aligned}
& X_{L}^{(3)}=X_{L}^{(3)}\left(P, T, X_{L}^{(2)}\right) \\
& X_{G}^{(1)}=X_{G}^{(1)}(P, T)
\end{aligned}
$$

Equation 3.17a describes the solubility of $\mathrm{NaCl}$ in water, which is considered only a function of temperature (see Chapter 2). Equation $3.17 \mathrm{~b}$ represents the solubility model presented in Section 2.4.2. Finally, the mass fraction of $\mathrm{H}_{2} \mathrm{O}$ in gas phase, modeled as an evaporation process, is represented by Equation $3.17 \mathrm{c}$.

6. Porosity is a function of pressure and solid saturation [one relationship].

$$
\phi=\phi\left(P, S_{S}\right)
$$

7. Overall thermal conductivity is a function of fluid phase saturations [one relationship].

$$
K=K\left(S_{G}, S_{L}\right)
$$

8. Tortuosity is function of porosity and phase saturation [two relationship].

$$
\begin{gathered}
\tau_{0, G}=\tau_{0, G}\left(\phi, S_{G}\right) \\
\tau_{0, L}=\tau_{0, L}\left(\phi, S_{L}\right)
\end{gathered}
$$

The previous 24 closure functions or independent relationships constitute the fundamental building blocks of the ECO2 module. Formulation of these functions was discussed in Chapter 2. 


\subsection{Numerical implementation}

\subsubsection{Space and time discretization}

The integral finite difference (IFD) method [Narasimhan and Witherspoon, 1976] is used to discretize the mass and energy balance Equation 3.1. In the IFD method, we introduce volume average as:

$$
\int_{V_{n}} M d V=V_{n} M_{n}
$$

where $M_{n}$ is the average value of $M$ over $V_{n}$. Surface integrals are approximated as a discrete sum of averages over surface segments $A_{n m}$ :

$$
\int_{\Gamma_{n}} \mathbf{F} \cdot \mathbf{n} d \Gamma=\sum_{m} A_{n m} F_{n m}
$$

where $A_{n m}$ is the interface area between grid blocks $n$ and $m$, and $F_{n m}$ is the average value of the (inward) normal component of $\mathbf{F}$ over $A_{n m}$ (see Figure 4.1).

After appropriate volume and surface area averages are taken in Equation 3.1, a set of first-order ordinary differential equations in time is obtained.

$$
\frac{d M_{n}^{(\kappa)}}{d t}=\frac{1}{V_{n}} \sum_{m} A_{n m} F_{n m}^{(\kappa)}+q_{n}^{(\kappa)}
$$

The summation is over all grids blocks $m$ that are connected to $n . F_{n m}^{(\kappa)}$ is the flux of component $\kappa$ between grid blocks $n$ and $m$. Moreover $F_{n m}^{(\kappa)}$ is the summation of fluxes over all mobile phases $(N P H)$.

$$
F_{n m}^{(\kappa)}=\sum_{\beta=1}^{N P H} F_{\beta, n m}^{(\kappa)}
$$




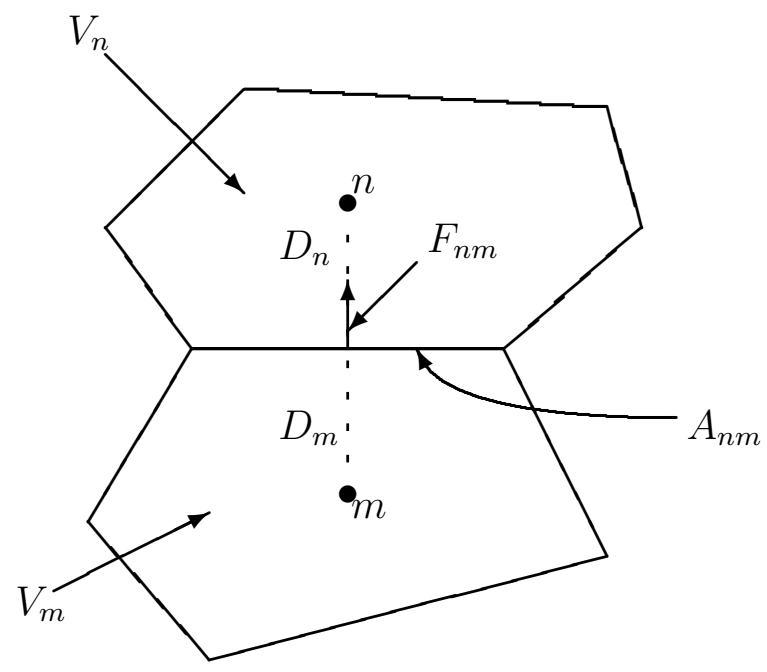

Figure 3.1: Space discretization in the integral finite difference method

Individual phase fluxes are expressed in terms of averages over parameters for elements $V_{n}$ and $V_{m}$. A finite difference approximation for the basic Darcy flux term (advective flux, Equation 3.4) is given by:

$$
F_{\beta, n m}=-k_{n m}\left(\frac{k_{r \beta} \rho_{\beta}}{\mu_{\beta}}\right)_{n m}\left(\frac{P_{\beta, n}-P_{\beta, m}}{D_{n m}}-\rho_{\beta, n m} g_{n m}\right)
$$

where the subscripts $(n m)$ indicate that the quantities $k_{n m},\left(\frac{k_{r \beta}}{\mu_{\beta}}\right)_{n m}$, and $\rho_{\beta, n m}$ (permeability, mobility and density, respectively) are evaluated at the interface between elements $m$ and $n$ using a suitable averaging technique (interpolation, harmonic weighting, upstream weighting). Finally, $D_{n m}$ is the distance between the nodal points $n$ and $m$, and $g_{n m}$ is the component of gravitational acceleration in the direction of $m$ to $n$.

Time is discretized fully implicitly as a first order finite difference, and the flux and sink/source terms on the right-hand side of Equation 3.23 are evaluated at the new time level, $t^{k+1}=t^{k}+\Delta t$. This ensures the numerical stability needed for an 
efficient calculation of multiphase flow [Peaceman, 1977]. The time-discretized version of Equation 3.23 can be written in terms of mass components and energy residuals as the following set of coupled non-linear, algebraic equations.

$$
R_{n}^{(\kappa), k+1}=M_{n}^{(\kappa), k+1}-M_{n}^{(\kappa), k}-\frac{\Delta t}{V_{n}}\left\{\sum_{m} A_{n m} F_{n m}^{(\kappa), k+1}+V_{n} q_{n}^{(\kappa), k+1}\right\}=0
$$

For each volume element (grid block) $V_{n}$ there are four equations $(\kappa=1,2,3,4)$ so that, in a flow domain with $N E L$ grid blocks, Equation 3.26 represents a total of $4 \times N E L$ coupled non-linear equations.

\subsubsection{Solution method}

The unknowns in Equation 3.26 are the $4 \times N E L$ independent primary variables $\left(x_{i} ; i=1, \cdots, 4 \times N E L\right)$ which are obtained by Newton-Raphson iteration. NewtonRaphson iteration is implemented in TOUGH2 as follows. A Taylor series expansion

of the residuals $R_{n}^{(\kappa), k+1}$ in equation 3.26 about an assumed solution $x_{i, p+1}$ (iteration step $p+1)$ is performed:

$$
R_{n}^{(\kappa), k+1}\left(x_{i, p+1}\right)=R_{n}^{(\kappa), k+1}\left(x_{i, p}\right)+\left.\sum_{i} \frac{\partial R_{n}^{(\kappa), k+1}}{\partial x_{i}}\right|_{p}\left(x_{i, p+1}-x_{i, p}\right)+\cdots=0
$$

Retaining only first order terms, we obtain a set of $4 \times N E L$ linear equations for the increments $\left(x_{i, p+1}-x_{i, p}\right)$ :

$$
-\left.\sum_{i} \frac{\partial R_{n}^{(\kappa), k+1}}{\partial x_{i}}\right|_{p}\left(x_{i, p+1}-x_{i, p}\right)=R_{n}^{(\kappa), k+1}\left(x_{i, p}\right)
$$

All terms $\partial R_{n} / \partial x_{i}$ represent entries of the Jacobian matrix that are evaluated by numerical differentiation. The system of simultaneous equations is solved with preconditioned conjugate gradient methods [Moridis and Pruess, 1998]. Iteration is continued until all residuals $R_{n}^{(\kappa), k+1}$ are reduced below a preset convergence tolerance. Typically, we demand $\left|R_{n}^{(\kappa), k+1} / M_{n}^{(\kappa), k+1}\right| \leq 10^{-5}$. 


\subsection{ECO2 module description}

For a non-isothermal system of three mass components, four independent thermodynamic parameters or primary variables are necessary to identify the thermodynamic state. The natural choice for a primary variable dealing with the heat balance equation is the system temperature $T$. The choice of the remaining primary variables must allow for a recognition of the phase composition of three-component mixtures of water, salt and $\mathrm{CO}_{2}$, and must be able to deal with the possibility that any of the three phases (liquid (aqueous), gas, solid) may appear or disappear in the course of a simulation. Phase changes and the choice of primary variables has been the topic of extensive research and continue to be the subject of debate [Crone et al., 2002; Miller et al., 1998]. We adopt the selection of primary thermodynamic variables implemented in the geothermal reservoir simulator TOUGH2-EWASG [Battistelli et al., 1997]. Depending on fluid phase conditions, the primary thermodynamic variables are chosen as follows.

- Single-phase fluid (only liquid, or only gas): $P, X_{s m}, X_{n}$, and $T$, where $P$ is pressure, $X_{s m}$ is the salt mass fraction $\left(X_{L}^{(2)}\right.$ or $\left.X_{G}^{(2)}\right)$ or solid saturation plus ten $\left(S_{S}+10\right), X_{n}$ is the $\mathrm{CO}_{2}$ mass fraction $\left(X_{L}^{(3)}\right.$ or $\left.X_{G}^{(3)}\right)$, and $T$ is temperature.

- Two-phase fluid (liquid and gas): $P_{G}, X_{s m}, S_{G}+10$, and $T$, where $P_{G}$ is gas pressure, $X_{s m}$ is the salt mass fraction $\left(X_{L}^{(2)}\right)$ or solid saturation plus ten $\left(S_{S}+\right.$ 10), $S_{G}+10$ is gas phase saturation plus ten, and $T$ is temperature.

The reason for having the solid saturations plus ten as a primary variable is that this allows to distinguish conditions where solid salt is present from those where is not. A similar reason follows for the third primary variables. 


\subsubsection{Program structure}

The equation of state module ECO2 is incorporated into the numerical simulator taking full advantage of TOUGH2's modular architecture MULKOM (see Figure 3.2). We use the term MULKOM to refer to the general architecture of the code. In fact, such an architecture was first implemented in a research code known as MULKOM in the early's 80s, and later formed the basis of TOUGH, a more specialized multiphase code for water-air-heat [Pruess, 1987]. Figure 3.3 shows how ECO2's equation of state (EOS) subroutine and all its satellites are linked into TOUGH2 (From Fujitsu Visual Analyzer $\left.{ }^{(}\right)$. EOS is responsible for handling phase diagnostic and obtaining thermophysical properties and constitutive relations from its satellites (See Figure 3.3 and Table 3.2).

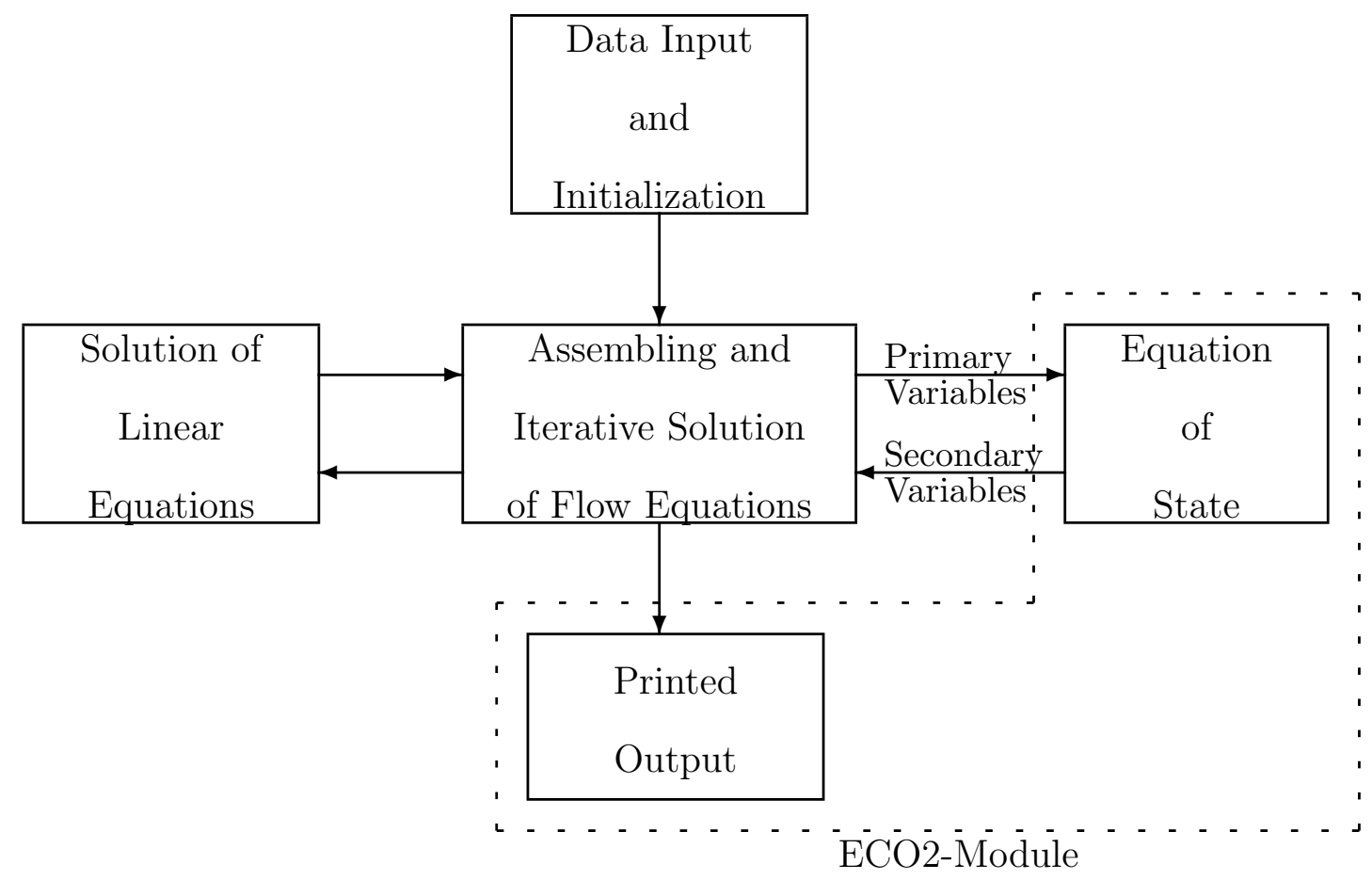

Figure 3.2: Modular Architecture MULKOM of TOUGH2-ECO2 (FromPruess et al. [1999]) 


\subsubsection{Phase diagnostic}

To check for possible phase change to two-phase condition, we test whether the "bubble pressure" of the fluid mixture exceeds the total pressure. If

$$
P_{b u b b l e}\left(T, X_{L}^{(2)}, X_{L}^{(3)}\right)>P
$$

then the gas phase appears and the appropriate primary variable is switched. For single-phase liquid conditions, the bubble pressure is given by

$$
P_{\text {bubble }}=P_{C O_{2}}\left(T, X_{L}^{(2)}, X_{L}^{(3)}\right)+P_{\text {sat }}\left(T, X_{L}^{(2)}\right)
$$

where $P_{\mathrm{CO}_{2}}$ is the $\mathrm{CO}_{2}$ partial pressure obtained from the solubility model describe in Chapter 2 (Subroutine HENRY), and $P_{\text {sat }}$ is the brine saturated pressure. For singlephase gas conditions, we calculate partial pressure $P_{v}$ of water vapor, and compare with saturated vapor pressure $P_{s a t}$. If $P_{v}>P_{\text {sat }}$, a liquid phase evolve.

Solid salt precipitation starts if the salt mass fraction in the liquid phase exceeds the halite equilibrium solubility $\left(X_{L}^{(2)}>X_{e q}^{(2)}(T)\right)$. If this occurs the primary variable is switched to $S_{S}+10$. Disappearance of the solid phase is recognized by $S_{S}<0$. Additional details about phase diagnostic is given by Battistelli et al. [1997] and Pruess and García [2002].

\subsubsection{Modeling of permeability reduction}

As mentioned before, the presence of salt in the system induces additional processes, particularly salt precipitation. The volume fraction of precipitated salt in the original pore space $\phi_{0}$ was termed "solid saturation" (Section 3.2.2) and denoted by $S_{S}$. A fraction $\phi_{0} S_{S}$ of reservoir volume is occupied by precipitate, while the remain- 
ing void space $\phi_{f}=\phi_{0}\left(1-S_{S}\right)$ is available for fluid phases; $\phi_{f}$ is referred to as "active flow porosity". The reduction of pore space reduces the permeability of the medium. The current version of ECO2 adopted the models for permeability reduction from the EWASG module [Battistelli et al., 1997]. The change of permeability due to porosity reduction is a complex process and a variety of models have been proposed in the literature Verma and Pruess [1988] and Pape et al. [1999]. Reduction in permeability depends not only on the overall reduction of the pore space but on pore space geometry and distribution of the precipitate. In general the overall relative change in permeability can be expressed as function of relative change in active flow porosity

$$
\frac{k}{k_{0}}=f\left(\frac{\phi_{f}}{\phi_{0}}\right) \equiv f\left(1-S_{S}\right)
$$

Additional details on the functional form of Equation 3.31 are provided in Verma and Pruess [1988], Battistelli et al. [1997], and Pruess et al. [1999]. 

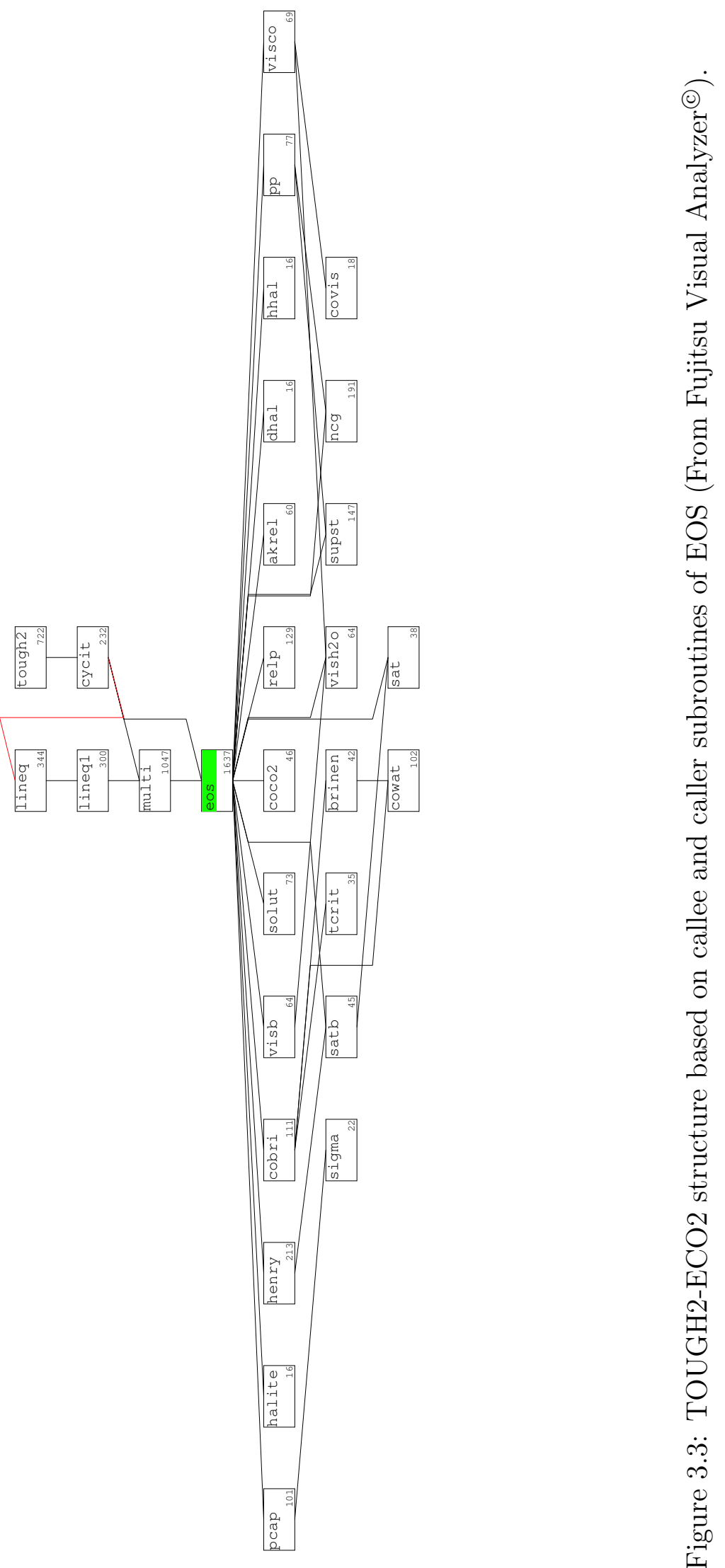
Table 3.2: Short description of subroutines in Figure 3.3

\begin{tabular}{|c|c|}
\hline Subroutine & Description \\
\hline EOS & Performs phase diagnostic \\
\hline & $\begin{array}{l}\text { Computes all thermophysical properties of gaseous, liquid, or two-phase } \\
\text { mixtures of } \mathrm{H}_{2} \mathrm{O}-\mathrm{NaCl}-\mathrm{CO}_{2} \text {. Calls the following satellite }\end{array}$ \\
\hline PCAP & $\begin{array}{l}\text { Computes capillary pressure as a function of liquid saturation } \\
\text { and temperature }\end{array}$ \\
\hline HALITE & Equilibrium halite solubility \\
\hline HENRY & $\begin{array}{l}\text { Calculates the phase partitioning of } \mathrm{CO}_{2} \text { between aqueous and gas phases } \\
\text { or } \mathrm{CO}_{2} \text { bubbling pressure }\end{array}$ \\
\hline COBRI & $\begin{array}{l}\text { Density and enthalpy of liquid brine as a function of pressure, } \\
\text { temperature, and salt mass fraction }\end{array}$ \\
\hline VISB & $\begin{array}{l}\text { Viscosity of brine as function of pressure, temperature, } \\
\text { and salt mass fraction }\end{array}$ \\
\hline SOLUT & Heat of solution of $\mathrm{CO}_{2}$ as function of temperature and salt mass fraction \\
\hline $\mathrm{COCO} 2$ & $\begin{array}{l}\text { Density of aqueous solution as function of pressure, temperature, } \\
\text { and } \mathrm{CO}_{2} \text { mass fraction }\end{array}$ \\
\hline RELP & Relative permeability for aqueous and gaseous phases \\
\hline AKREL & Permeability reduction factor as a function porosity reduction \\
\hline DHAL & Halite density as function of pressure and temperature \\
\hline HHAL & Halite specific enthalpy as function of temperature \\
\hline $\mathrm{PP}$ & $\begin{array}{l}\text { Vapor partial pressure, density and internal energy as function of } \\
\text { pressure, temperature, and mass fraction }\end{array}$ \\
\hline VISCO & Viscosity of vapor-air mixtures \\
\hline VISGAS & $\begin{array}{l}\text { Viscosity of } \mathrm{CO}_{2} \text { as function of pressure and temperature, overwritten } \\
\text { in NCG with values from table }\end{array}$ \\
\hline SIGMA & Surface tension of water as function of temperature \\
\hline SATB & $\begin{array}{l}\text { Vapor pressure of brine as a function of pressure, temperature, } \\
\text { and salt mass fraction }\end{array}$ \\
\hline TCRIT & Critical temperature of $\mathrm{NaCl}$ solutions as a function of salinity \\
\hline BRINEN & $\begin{array}{l}\text { Deviation of brine enthalpy as function of temperature } \\
\text { and salt mass fraction }\end{array}$ \\
\hline VISH2O & $\begin{array}{l}\text { Viscosity of liquid water or vapor as function of pressure, temperature, } \\
\text { and density (ASME, 1977) }\end{array}$ \\
\hline SUPST & Vapor density and internal energy as function of pressure and temperature \\
\hline NCG & Specific enthalpy, density, and viscosity of gaseous $\mathrm{CO}_{2}$ \\
\hline COVIS & Coefficient for gas phase viscosity calculation \\
\hline COWAT & $\begin{array}{l}\text { Liquid water density and internal energy as function of } \\
\text { pressure and temperature }\end{array}$ \\
\hline SAT & Steam table equation: saturation pressure as function of temperature \\
\hline
\end{tabular}




\section{Chapter 4}

\section{Code verification and applications}

\subsection{Introduction}

Carbon dioxide can be sequestered in aquifers by three principal mechanisms. First, $\mathrm{CO}_{2}$ can be trapped under a low-permeability caprock, similar to the way that natural gas is trapped in reservoirs or stored in aquifers. This mechanism, called $h y$ drodynamic trapping, relies on the physical displacement of pore fluids. Second, pore fluids can accumulate dissolved $\mathrm{CO}_{2}$ through aqueous or solubility trapping. Finally, dissolved $\mathrm{CO}_{2}$ can react with divalent cations to form carbonate mineral precipitates. This mechanism is called mineral trapping. The results of analyzes of hydrodynamic and solubility trapping using TOUGH2/ECO2 are presented in this chapter. The discussion emphasizes the physics of multiphase flow in one- and two-dimensional domains. The aim here is to provide a better understanding of the fundamental physical flow processes that are related to $\mathrm{CO}_{2}$ disposal into brine formations.

Even though the issues of chemical and mechanical effects are neglected herein, earlier versions of TOUGH2/ECO2 have been successfully incorporated into numerical simulators that address coupled hydro-chemical and hydro-mechanical processes.

Pruess et al. [2003] and Xu et al. [2003] performed reactive geochemical simulations to 
study mineral trapping for $\mathrm{CO}_{2}$ disposal in deep formations, and a study of caprock hydro-mechanical changes associated with $\mathrm{CO}_{2}$ injection into brine formation was presented by Rutqvist and Tsang [2002]. Finally, actual field applications in heterogeneous formations, have been carried out with an earlier version of TOUGH2/ECO2 by Doughty and Pruess [2003].

An outline of this chapter is as follows. In section 4.2, the basic problem of $\mathrm{CO}_{2}$ injection into a radially symmetric brine aquifer is presented. Because the simplified radial flow allows a similarity solution, this problem will be used to check that the governing equations of the mathematical model have been correctly implemented, and that the numerical solution confirms the similarity property. Accordingly, radial flow from an injection $\mathrm{CO}_{2}$ well serves as our "code verification" problem. A more complex flow problem is considered in Section 4.3, representing the $\mathrm{CO}_{2}$ injection project at the Sleipner Vest Field in the Norwegian sector of the North Sea. Finally, issues regarding enhanced $\mathrm{CO}_{2}$ dissolution by convective mixing are presented in Section 4.4 .

\subsection{Radial flow from a $\mathrm{CO}_{2}$ injection well}

Radial flow and pressure behavior of an injection well is a problem of fundamental importance in the reservoir engineering of $\mathrm{CO}_{2}$ aquifer disposal. An idealized $\mathrm{CO}_{2}$ injection well problem is solved to examine two-phase flow of carbon dioxide and water for simplified flow geometry and medium properties. The aquifer into which injection is made is assumed infinite-acting, homogeneous, and isotropic. Gravity and inertial effects are neglected, injection is made at a constant rate, and flow is assumed 1-D radial (line source). The list of processes being studied includes:

- Two-phase flow of $\mathrm{CO}_{2}$ and $\mathrm{H}_{2} \mathrm{O}$ subject to relative permeability and capillary effects. 
- Change of fluid density, viscosity, and $\mathrm{CO}_{2}$ solubility with pressure and salinity.

- Formation dry-out with precipitation of salt.

- Permeability reduction due to salt precipitation.

An important advantage of the simplified radial flow is that it allows a similarity solution. The solution depends on radial distance $R$ and time $t$ only through the similarity variable $\xi=R^{2} / t$. This holds true even when taking into account all the non-linearities due to PVT properties and due to two-phase flow [Doughty and Pruess, 1992; O'Sullivan, 1981]. In radial coordinates the non linear partial differential equations governing mass and energy transport (Equation 3.1) can be reduced to simpler ordinary differential equations by the use of the similarity variable $R^{2} / t$. Based on this variable substitution, known as the Boltzman transformation in heat conduction problems, the resulting system of non-linear ODE's can be solved numerically by using standard techniques [O'Sullivan, 1981]. The space discretization employed for finite difference simulation will violate the rigorous $R^{2} / t$ invariance, so that the similarity property will be maintained only approximately. Accuracy of the numerical simulation can be checked by plotting the results as a function of the similarity variable $R^{2} / t$.

\subsubsection{Definition of the problem and input data}

Specifications for the 1-D radial flow problem were chosen representative of conditions that may be encountered in brine aquifers at a depth of order $1.2 \mathrm{~km}$. The aquifer is assumed to have salinity of 10 -wt \% dissolved $\mathrm{NaCl}$, and initial pressure and temperature conditions of $P=120 \mathrm{bar}$ and $T=45^{\circ} \mathrm{C}$. Problem parameters are summarized in Table 4.1. The numerical grid extends to a large distance of $100 \mathrm{~km}$, so that the system acts as infinite for the time period simulated (10,000 days, 27.38 years). The well is modeled as a circular grid element of $R=0.3 \mathrm{~m}$ radius into which 
$\mathrm{CO}_{2}$ is injected uniformly at a constant rate of $100 \mathrm{~kg} / \mathrm{s}$, which corresponds to the $\mathrm{CO}_{2}$ emission rate of a 288-MWe (MegaWatt electric) coal-fired power plant [Hitchon, 1996].

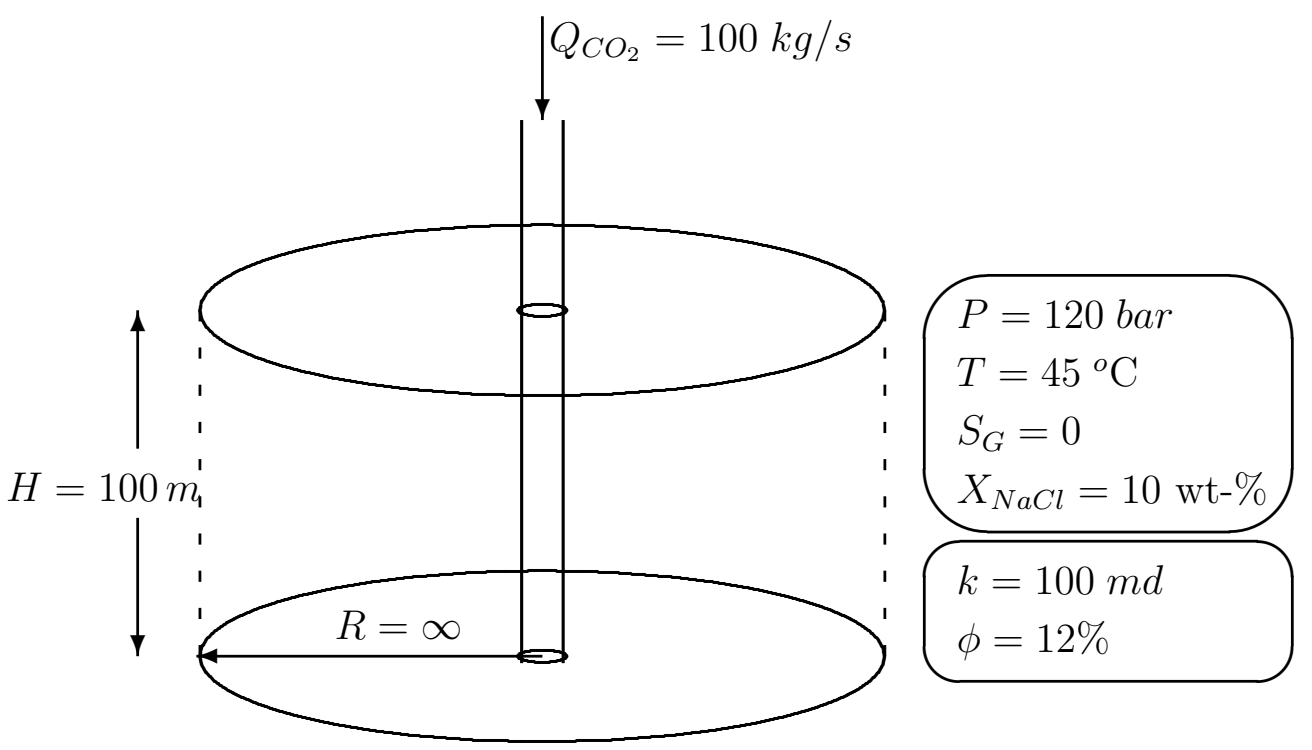

Figure 4.1: Schematic of radial flow from a $\mathrm{CO}_{2}$ injection well

\subsection{2 $\mathrm{CO}_{2}$ injection into a radial aquifer (no salinity)}

Figure 4.2 shows the evolution of the gas saturation front up to a time of 10,000 days. Figure 4.3 shows the results for pressure as a function of the similarity variable. Simulated results are presented for four different times $(t=30,100,1000,10000$ days) and two fixed locations $(R=25.25$, and $1011 \mathrm{~m})$. The agreement is good, confirming the similarity property of the numerical solution. Figures 4.4 and 4.5, show simulated results for gas saturation and dissolved $\mathrm{CO}_{2}$ mass fraction plotted as a function of the similarity variable. Gas saturation results show three distinct regions emerging from the $\mathrm{CO}_{2}$ injection process. The first region, $R^{2} / t \leq 5 \times 10^{-7} \mathrm{~m}^{2} / \mathrm{s}$, corresponds to a zone where complete dry-out of aqueous phase has occurred. This region is 
Table 4.1: Hydrologic parameters for one-dimensional radial flow simulations

\begin{tabular}{|c|c|}
\hline Permeability & $k=10^{-13} m^{2}$ \\
\hline Porosity & $\Phi=0.12$ \\
\hline Pore Compressibilty & $c=4.5 \times 10^{-10} \mathrm{~Pa}^{-1}$ \\
\hline Aquifer thickness & $100 \mathrm{~m}$ \\
\hline \multicolumn{2}{|l|}{ Relative Permeability } \\
\hline \multicolumn{2}{|l|}{ liquid: van Genuchten [1980] } \\
\hline$k_{r l}=\sqrt{S^{*}}\left[1-\left(1-\left[S^{*}\right]^{1 / m}\right)^{m}\right]^{2}$ & $S^{*}=\left(S_{l}-S_{l r}\right) /\left(1-S_{l r}\right)$ \\
\hline irreducible water saturation & $S_{l r}=0.30$ \\
\hline exponent & $m=0.457$ \\
\hline \multicolumn{2}{|l|}{ gas: Corey [1954] } \\
\hline$k_{r g}=(1-\hat{S})^{2}\left(1-\hat{S}^{2}\right)$ & $\hat{S}=\frac{\left(S_{l}-S_{r}\right)}{\left(1-S_{l r}-S_{g r}\right)}$ \\
\hline irreducible gas saturation & $S_{g r}=0.05$ \\
\hline \multicolumn{2}{|l|}{ Capillary pressure } \\
\hline \multicolumn{2}{|l|}{ van Genuchten function } \\
\hline$P_{c a p}=-P_{0}\left(\left[S^{*}\right]^{-1 / m}-1\right)^{1-m}$ & $S^{*}=\left(S_{l}-S_{l r}\right) /\left(1-S_{l r}\right)$ \\
\hline irreducible water saturation & $S_{l r}=0.0$ \\
\hline exponent & $m=0.457$ \\
\hline strength coefficient & $P_{0}=19.61 \mathrm{kPa}$ \\
\hline \multicolumn{2}{|l|}{ Initial Conditions } \\
\hline Salinity & 10 wt.- $\%$ \\
\hline Pressure & 120 bar \\
\hline Temperature & $45^{\circ} \mathrm{C}$ \\
\hline Injection (constant rate) & $100 \mathrm{~kg} / \mathrm{s}$ \\
\hline
\end{tabular}

followed by an intermediate region extending to $R^{2} / t \approx 10^{-2} \mathrm{~m}^{2} / \mathrm{s}$, where liquid and gas phase coexists. Finally, there is an outer region with $R^{2} / t \geq 10^{-2} \mathrm{~m}^{2} / \mathrm{s}$ in which liquid phase conditions prevail. 


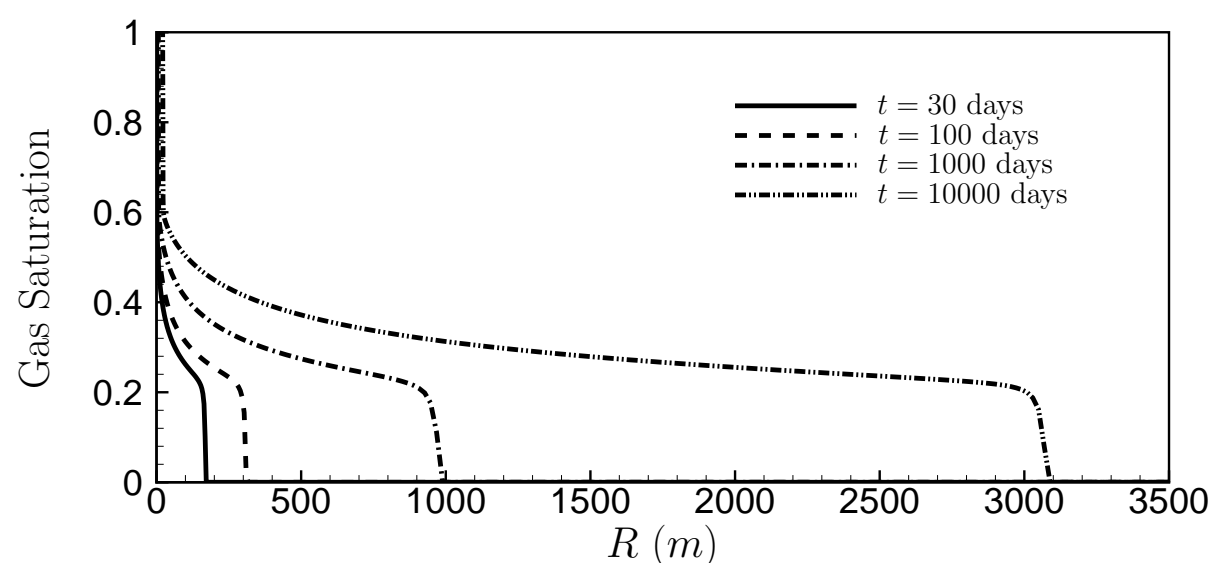

Figure 4.2: Simulated gas saturation front (no salinity).

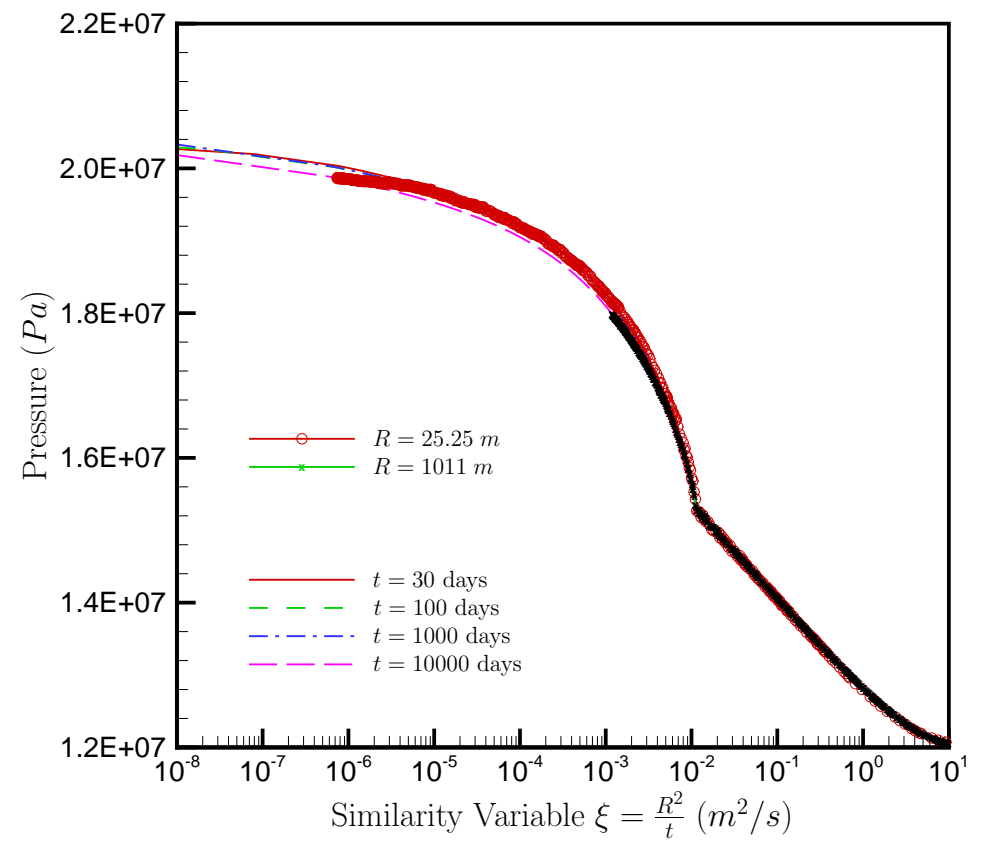

Figure 4.3: Simulated pressure (no salinity). 


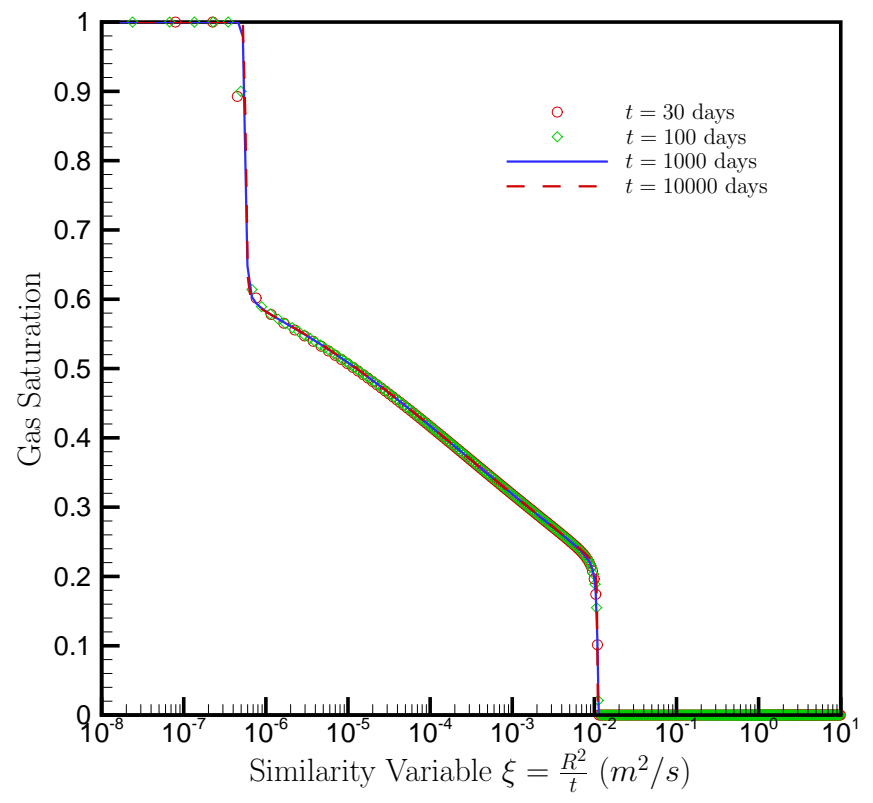

Figure 4.4: Simulated gas saturation (no salinity).

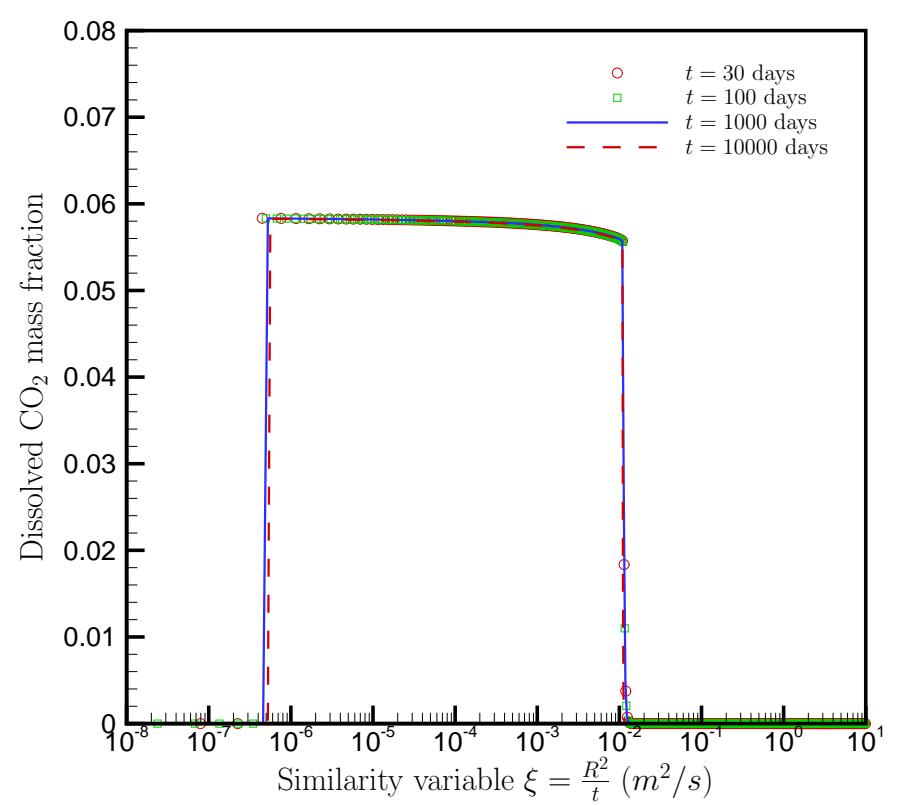

Figure 4.5: Simulated dissolved $\mathrm{CO}_{2}$ mass fraction (no salinity). 


\subsection{3 $\mathrm{CO}_{2}$ injection into a radial saline aquifer}

The presence of salt in the system induces additional processes, particularly salt precipitation near the injection well. The volume fraction of precipitated salt in the original pore space $\phi_{0}$ is termed "solid saturation", and denoted by $S_{S}$. A fraction $\phi_{0} S_{S}$ of reservoir volume is occupied by precipitate, while the remaining void space $\phi_{f}=\phi_{0}\left(1-S_{S}\right)$ is available for fluid phases; $\phi_{f}$ is referred to as "active flow porosity". The reduction of pore space reduces the permeability of the medium. The change of permeability due to porosity reduction is a complex process and a variety of models have been proposed in the literature [Pape et al., 1999; Verma and Pruess, 1988]. We use the tubes-in-series model to represent permeability changes from precipitation which includes effect of pore throats [Verma and Pruess, 1988]. Permeability reduction is given by

$$
\frac{k}{k_{0}}=\theta^{2} \frac{1-f+f / \omega^{2}}{1-f+f[\theta /(\theta+\omega-1)]^{2}}
$$

Here,

$$
\theta=\frac{1-S_{S}-\phi_{r}}{1-\phi_{r}}
$$

depends on the fraction $1-S_{S}$ of original porosity which remains available for fluid flow, and on a parameter $\phi_{r}$ which denotes the fraction of original porosity at which permeability is reduced to zero. $f$ is the fractional length of the pore bodies, and the parameter $\omega$ is

$$
\omega=1+\frac{1 / f}{1 / \phi_{r}-1}
$$

Therefore, only two independent geometric parameters need to be specified to calculate permeability reduction using equation 4.1 . With $\phi_{r}=f=0.8$, the results 
show that a modest amount of precipitation of $S_{S}=2 \%$ produces a reduction in permeability of $18 \%$.

Simulated results (Figures 4.6, 4.7, and 4.8) show that the overall behavior is very similar to the simulation run without salinity. As expected, the three distinct regions developed during $\mathrm{CO}_{2}$ injection occur at about the same $R^{2} / t$ locations. A $8 \%$ higher pressure buildup occurs in the vicinity of the injection well, due to larger viscosity of brine compared to fresh water. Comparing Figure 4.8 with Figure 4.5 it is seen that dissolution of $\mathrm{CO}_{2}$ in the aqueous phase is reduced by $40 \%$ due to salinity effects ("salting out").

The figures for pressure, saturation and dissolved $\mathrm{CO}_{2}$ mass fraction demonstrate that the similarity property is well maintained. The results for solid saturation show considerable variations at small values of $R^{2} / t$ where discretization errors are significant (Figure 4.9). Salt precipitation occurs only in the vicinity of the injection well (first 20 meters). In order to reduce discretization errors when calculating solid salt saturation, we performed another simulation with a finer grid, using small increments of constant $\delta R=0.05 \mathrm{~m}$ near the injection point. This prevents large jumps in solid saturation at elements near the injection well, and provides excellent preservation of the similarity property (Figure 4.10). 


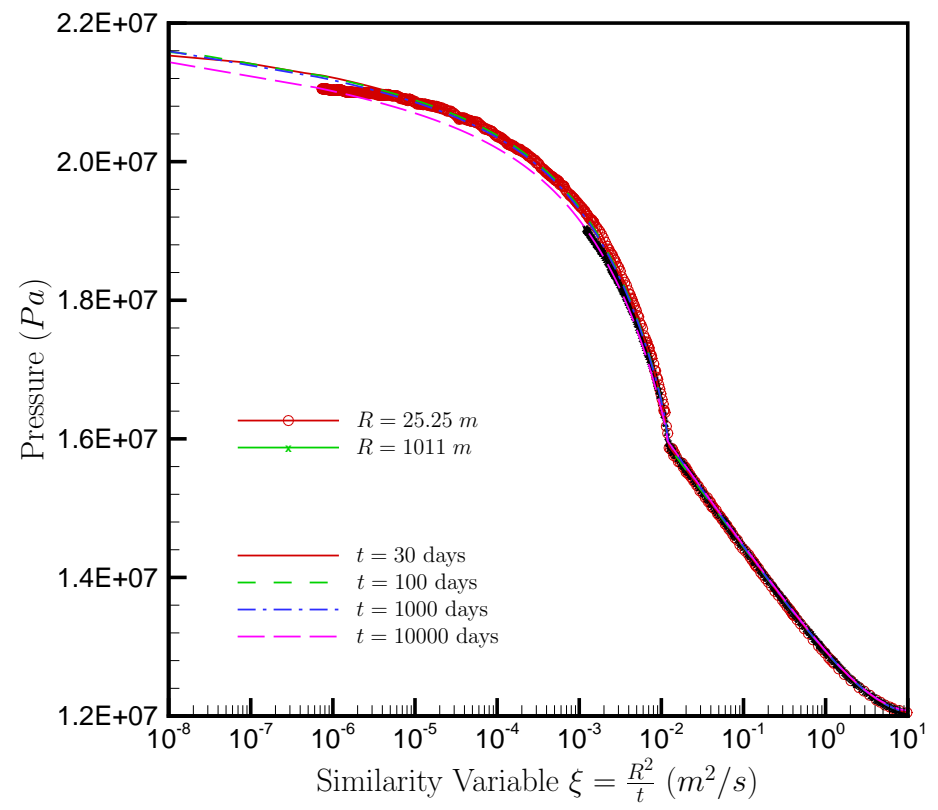

Figure 4.6: Simulated pressure (10 wt.- $\%$ salinity).

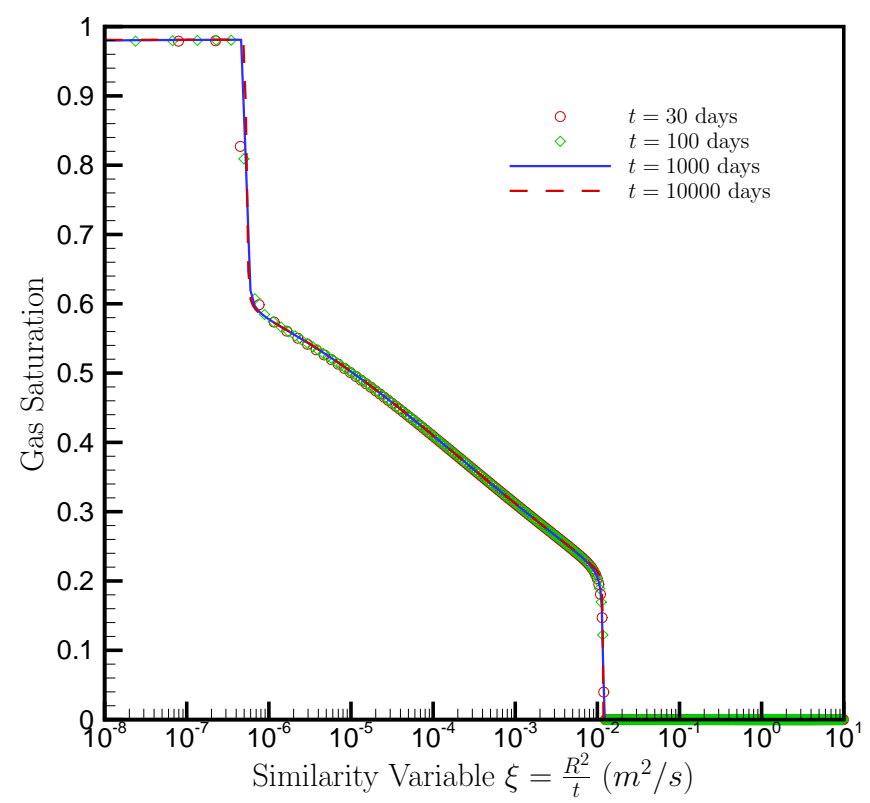

Figure 4.7: Simulated gas saturation (10 wt.- \% salinity). 


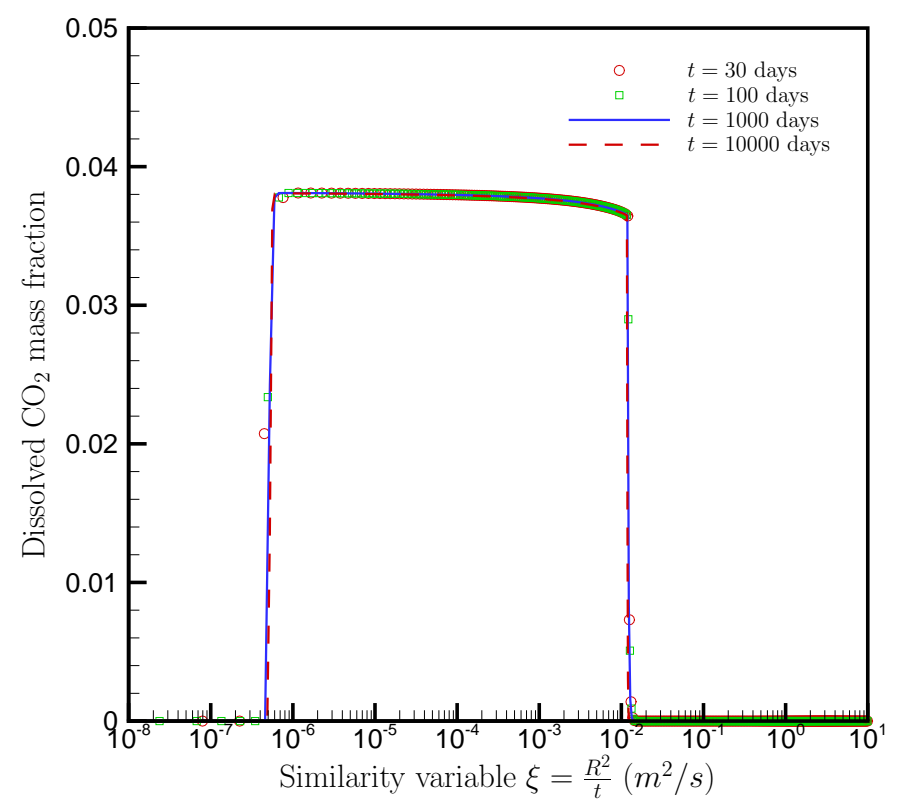

Figure 4.8: Simulated dissolved $\mathrm{CO}_{2}$ mass fraction (10 wt.- $\%$ salinity).

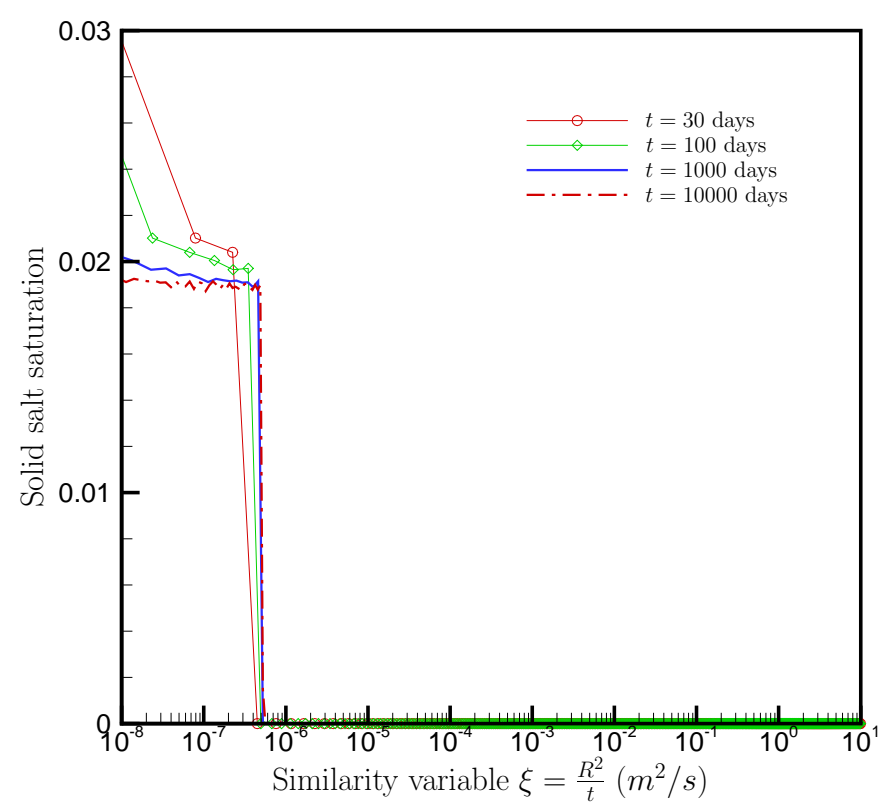

Figure 4.9: Simulated solid salt saturation (10 wt.- \% salinity). 


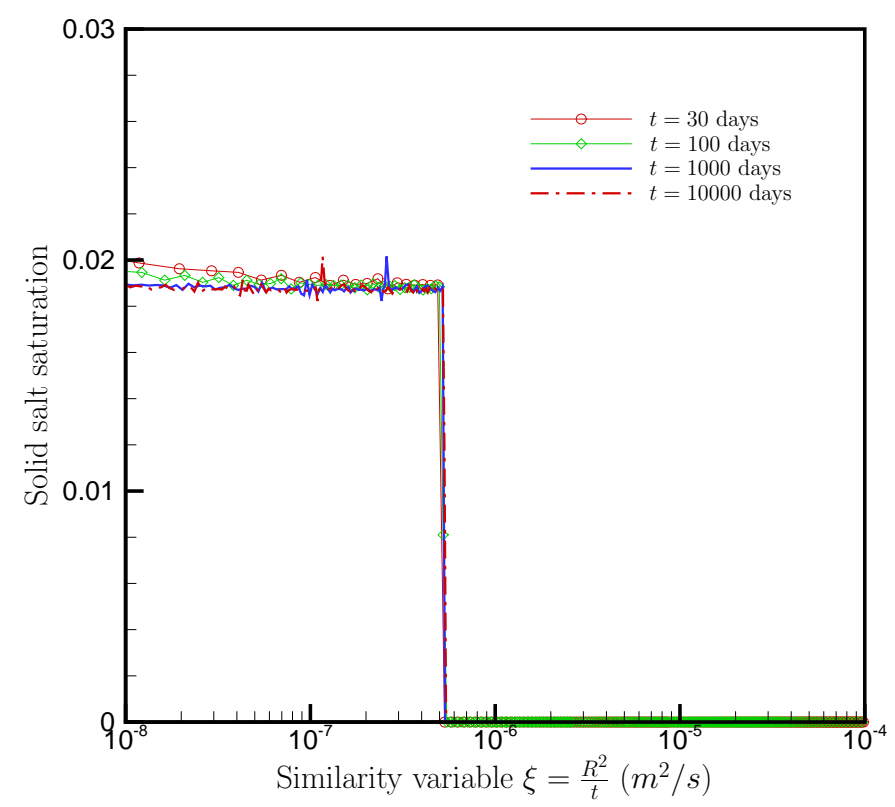

Figure 4.10: Simulated solid salt saturation using a finer grid near the injection well

\section{3 $\mathrm{CO}_{2}$ injection into a 2-D layered brine forma- tion}

This problem was designed after the $\mathrm{CO}_{2}$ injection project at the Sleipner Vest field in the Norwegian sector of the North Sea, where approximately $10^{6}$ tonnes of $\mathrm{CO}_{2}$ per year are injected through a horizontal well into sands of the Utsira formation. Figure 4.11 shows a schematic representation of the Sleipner storage system. Well data and seismic data prior to the injection shows that the sand is divided by nearly horizontal, discontinuous shales [Lindeberg et al., 2000]. Time lapse surveys show that the transport at Sleipner is dominated by buoyancy effects and that shale layers control vertical migration of $\mathrm{CO}_{2}$. The list of processes being studied includes: 
- Gravity-driven advection in response to strong vertical and lateral density gradients induced by injection of $\mathrm{CO}_{2}$ into saline aquifers.

- Change of fluid density, viscosity, and $\mathrm{CO}_{2}$ solubility with pressure and salinity.

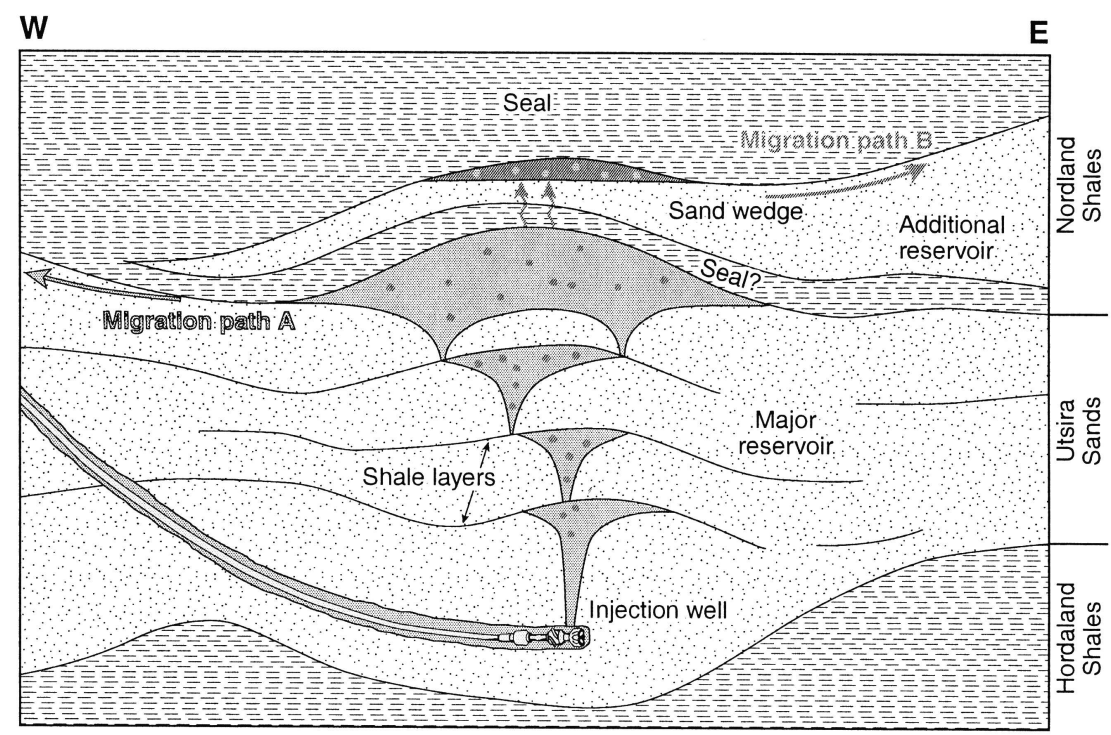

Figure 4.11: Schematic representation of the Sleipner storage system (From Zweigel et al. [2000]).

\subsubsection{Definition of the problem and input data}

The simplified 2-D vertical section modeled is presented in Figure 4.12. The system geometry and problem specifications are taken from the code intercomparison study designed by Lawrence Berkeley National Laboratory (LBNL) in the framework of the GeoSeq project [Pruess et al., 2002] (see Table 4.2). The system is idealized as a two-dimensional symmetric domain perpendicular to the horizontal injection well. The thickness of the formation at the injection site is 184 meters. The injection point is 940 meters below the sea floor, while the ocean depth at the site is 80 meters. The formation is assumed to consist of four lower permeability shale units 3 meters thick which are distributed within the high permeability sand. Each shale unit is 
separated by 30 meters. The well is 30 meters below the lowest shale unit, while the bottom of the aquifers is another 22 meters below the well. The vertical boundary at 6,000 meters from the injection well is fixed at hydrostatic pressure, the remaining boundaries are no flux.

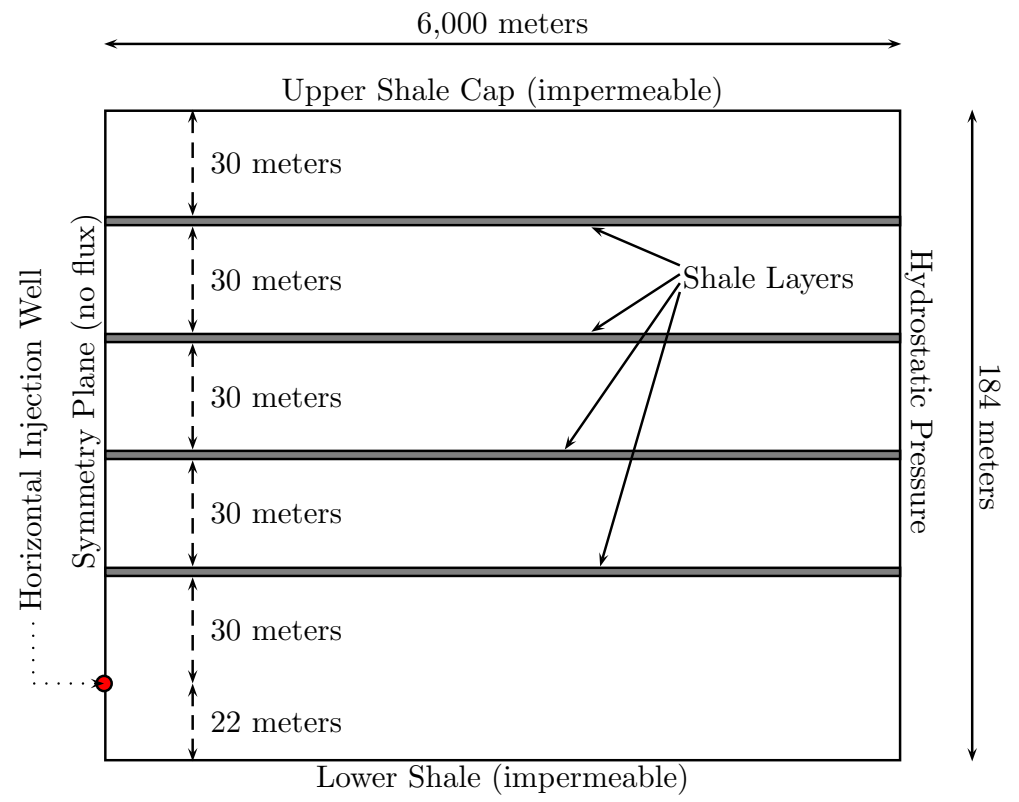

Figure 4.12: Simplified 2-D vertical section of the Sleipner storage system

The grid was designed to provide "adequate" spatial resolution near the injection well, and near the shale layers. Grid intervals in the horizontal direction (x-direction) start with 1 meter increments near the well and gradually increase to 250 meter increments at the hydrostatic pressure boundary $(6,000 \mathrm{~m}$ from the injection well). The vertical grid increment (z-direction) for layers below, above, and at the well is $1 \mathrm{~m}$. The shale layers are represented by two grid layers of $1.5 \mathrm{~m}$ height, with 2 layers of $1.5 \mathrm{~m}$ height overlying and underlying the shales. The thickness of the grid (y-direction) is $1 \mathrm{~m}$. The overall domain is discretized into 3,445 grid blocks (53 horizontal, 65 vertical). 
Table 4.2: Hydrologic parameters for two-dimensional layered brine formation

Permeability

Porosity

Aquifer thickness
Sands: $k=3 \times 10^{-12} m^{2}$; Shales: $k=10^{-14} m^{2}$

Sands: $\Phi=0.35$; $\underline{\text { Shales: }} \Phi=0.1025$

$184 m$

Relative Permeability

liquid: van Genuchten [1980]

$\overline{k_{r l}=} \sqrt{S^{*}}\left[1-\left(1-\left[S^{*}\right]^{1 / m}\right)^{m}\right]^{2} \quad S^{*}=\left(S_{l}-S_{l r}\right) /\left(1-S_{l r}\right)$

irreducible water saturation

$S_{l r}=0.20$

exponent

$m=0.400$

gas: van Genuchten [1980]

$k_{r g}=\sqrt{S_{g}^{*}}\left[1-\left(1-\left[S_{g}^{*}\right]^{1 / m}\right)^{m}\right]^{2} S_{g}^{*}=\left(S_{g}-S_{g r}\right) /\left(1-S_{g r}\right)$

irreducible gas saturation

$S_{g r}=0.05$

exponent

$m=0.400$

Capillary pressure

van Genuchten [1980]

$P_{\text {cap }}=-P_{0}\left(\left[S^{*}\right]^{-1 / m}-1\right)^{1-m} \quad S^{*}=\left(S_{l}-S_{l r}\right) /\left(1-S_{l r}\right)$

irreducible water saturation $\quad S_{l r}=0.20$

exponent $\quad m=0.400$

strength coefficient $\quad$ Sand: $P_{0}=3.58 \mathrm{kPa}$; Shale: $P_{0}=62.0 \mathrm{kPa}$

Initial Conditions

Salinity

3.2 wt. $-\%$

Pressure

Hydrostatic

Temperature

$37^{\circ} \mathrm{C}$

Injection (constant rate)

$0.1585 \mathrm{~kg} / \mathrm{s}$ 


\subsection{2 $\quad \mathrm{CO}_{2}$ injection results}

The problem was analyzed in several steps to first obtain the initial and boundary conditions, and then inject $\mathrm{CO}_{2}$ according to specifications. Initial conditions are generated in two stages by gravity equilibration simulation. The first simulation involved just the boundary at 6,000 meters. Thermodynamic properties are specified as given by Table 4.2. Pressure is held constant at $P=110$ bar at the elevation of the injection node $(22 \mathrm{~m})$ and the system is run to gravity. Then, a second simulation with the complete two-dimensional grid was performed maintaining the 1-D gravity equilibrium as a boundary.

Figure 4.13 and 4.14 show contours of gas saturation and $\mathrm{CO}_{2}$ mass fraction dissolved in the aqueous phase after one year and two years of $\mathrm{CO}_{2}$ injection respectively. The shale layers control the vertical migration of $\mathrm{CO}_{2}$, causing the plume to spread laterally. Large gas saturations beneath the shale layers show the importance of buoyancy effects. $\mathrm{CO}_{2}$ mass fraction dissolved in the aqueous phase ranges from $5-6 \%$ in the two-phase zone to smaller values beyond the two-phase region. 

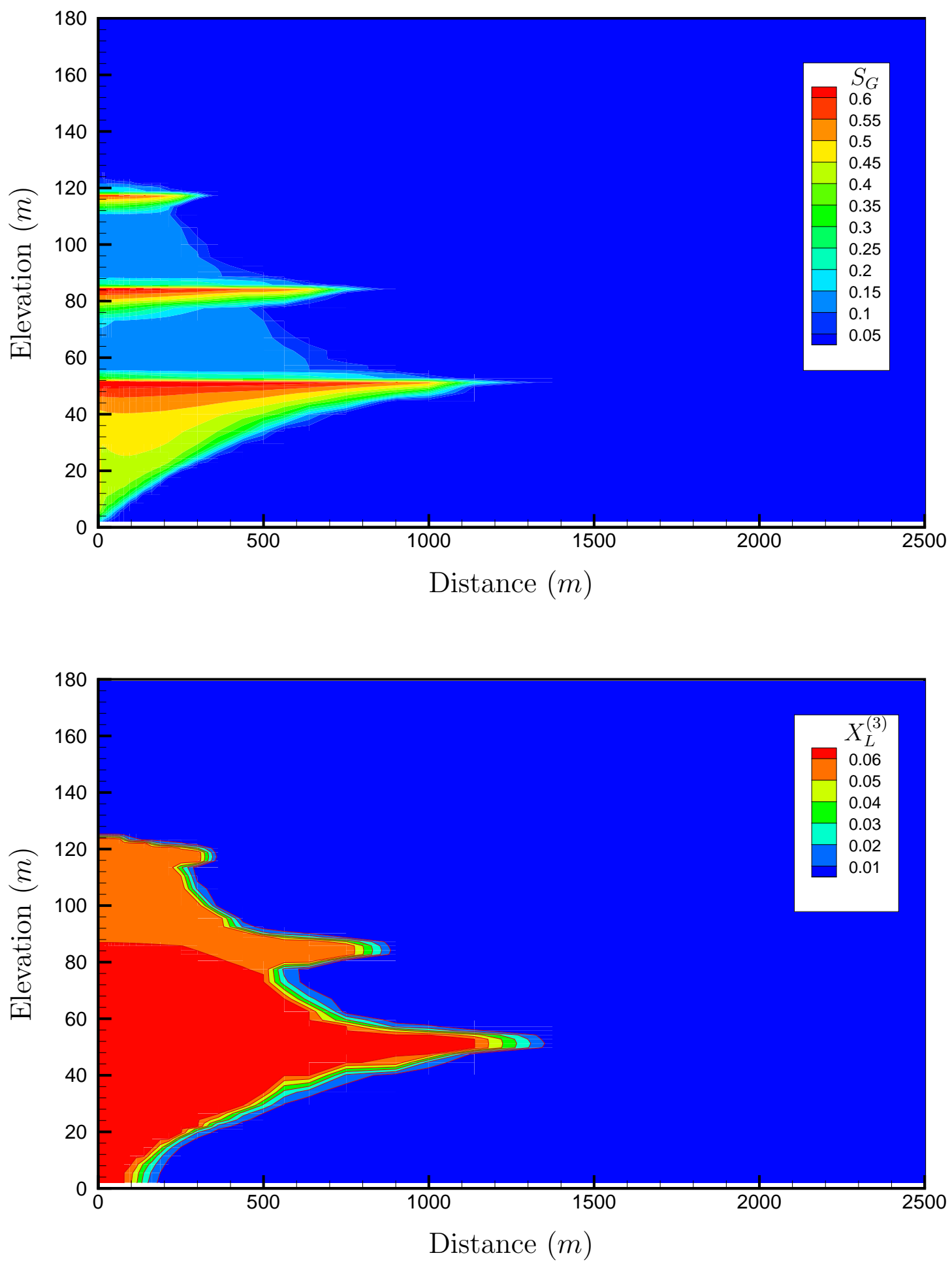

Figure 4.13: Simulated gas saturation (top) and dissolved $\mathrm{CO}_{2}$ (bottom) after 1 year. 

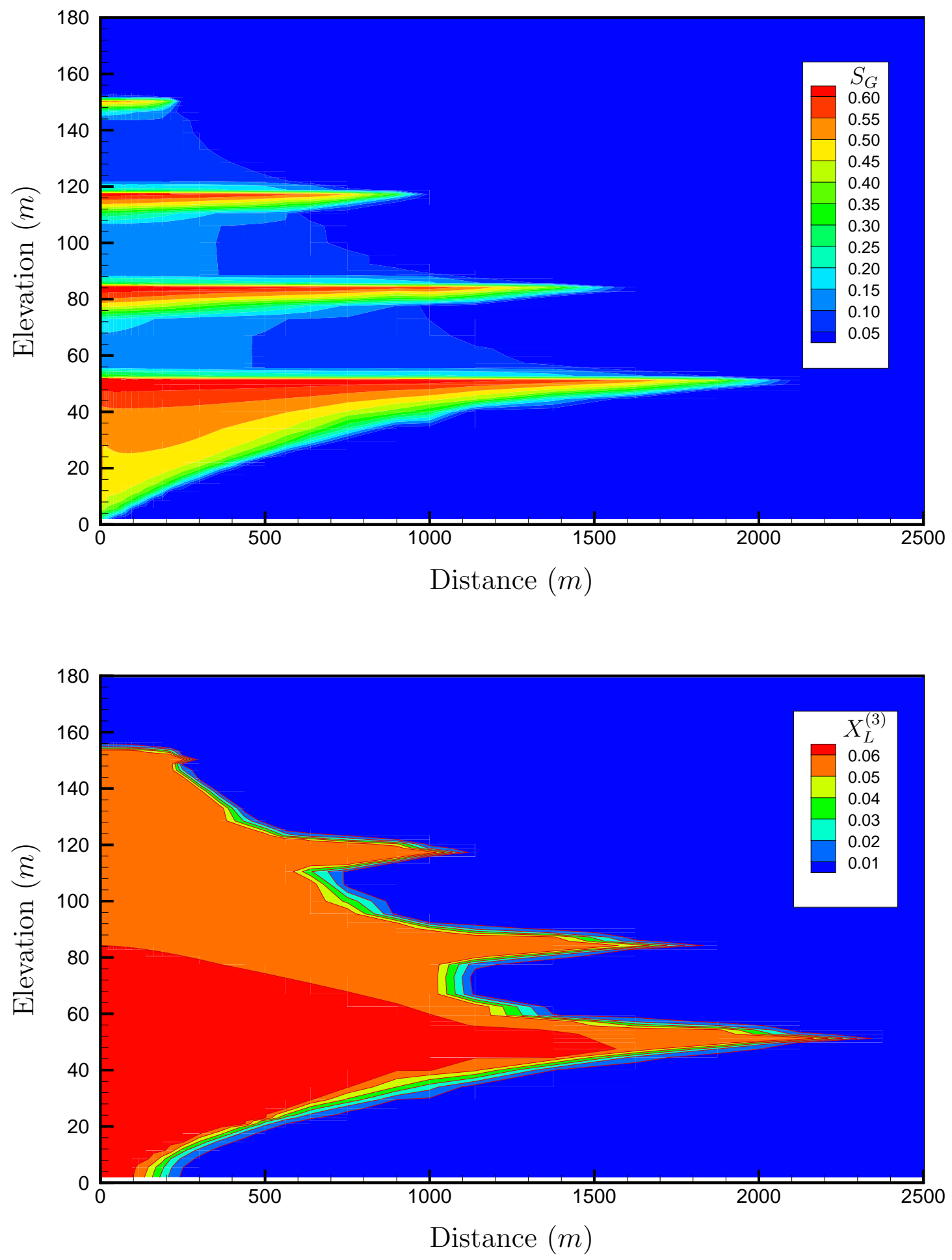

Figure 4.14: Simulated gas saturation (top) and dissolved $\mathrm{CO}_{2}$ (bottom) after 2 years. 


\subsection{Enhanced $\mathrm{CO}_{2}$ dissolution}

Disposal of $\mathrm{CO}_{2}$ into geological formations requires, among other conditions, the presence of a regional caprock for confinement. Under supercritical conditions, $\mathrm{CO}_{2}$ is less dense than water and would tend to migrate to the top of the formation as was demonstrated in the previous section. After a relatively long time (decades after ending injection) almost all gas would be stored at the top, underlying the caprock. Because the density of $\mathrm{CO}_{2}$ saturated water is slightly higher than the density of pure water, density-driven flow will occur enhancing the dissolution process by convective mixing. In this particular case the heavier $\mathrm{CO}_{2}$ saturated water will flow downward and will be replaced by water with lesser $\mathrm{CO}_{2}$ content. In such flow problems, numerical simulation capabilities that take into account variable density brines are crucial [Oldenburg and Pruess, 1995]. The simulator TOUGH2/ECO2 considers the effect of $\mathrm{CO}_{2}$ content on brine density through a correlation for the partial molar volume of $\mathrm{CO}_{2}$ in water and the calculation of the corresponding aqueous solution density (see Section 2.5). In typical underground storage conditions, $\mathrm{CO}_{2}$ saturated brine is approximately $10 \mathrm{~kg} / \mathrm{m}^{3}$ denser than brine with no $\mathrm{CO}_{2}$. Even with this small increment the $\mathrm{CO}_{2}$ saturated brine creates a density instability, and on long time scales this causes convective mixing, greatly increasing the overall rate of dissolution compared to a purely diffusive mechanism. The list of processes studied includes:

- Diffusive transport of $\mathrm{CO}_{2}$ in the aqueous phase.

- Change of aqueous phase density with $\mathrm{CO}_{2}$ content, pressure and salinity.

- Gravity driven flow and convective mixing. 


\subsubsection{Definition of the problem and input data}

The role of convective mixing on $\mathrm{CO}_{2}$ dissolution is explored here by simulating density instabilities in a simple 2-D flow geometry (500 m wide and $400 \mathrm{~m}$ vertically). Initial conditions before gravity equilibration involve a uniform two-phase layer with constant $50 \%$ gas saturation (10 m thick), pressure of $180 \mathrm{bar}$, salinity of 3.2-wt $\%$, and temperature of $50{ }^{\circ} \mathrm{C}$. Problem parameters are summarized in Table 4.3. Gridding in the horizontal direction is uniform with 50 increments of 10 meters. The grid in the vertical direction is refined at the top 20 meters ( $0.5 \mathrm{~m}$ increments) and gradually coarsened towards the bottom. The overall domain is discretized into 6,000 grid blocks (50 horizontal, 120 vertical), and gridding is considered rather coarse. In fact, a slightly finer grid in the horizontal direction produced some differences in the plume patterns that will be presented in the following section. Nevertheless, the role of convective mixing in $\mathrm{CO}_{2}$ dissolution can appropriately be studied using this coarse grid. In order to obtain a more meaningful quantitative description, issues of grid convergence, boundary conditions and domain size need to be addressed.

\subsubsection{Simulation Results}

Figure 4.15 shows the evolution of gravity driven fingers at $t=100,300,500$, 800, 1200, and 1800 years, when 12, 13, 16, 21, 24, and $27 \%$ of $\mathrm{CO}_{2}$ has dissolved. Ennis-King and Paterson [2003] provided an excellent review of the theory of convective stability in porous media. The mathematical formulation of the solute-driven convection is related to the case of temperature-driven convection which has been extensively studied. From scaling analysis of transient heat problems, Ennis-King and Paterson [2003] proposed the following relation for the critical time $t_{c}$ at which 
Table 4.3: Hydrologic parameters for convective mixing simulations

\begin{tabular}{ll}
\hline \hline Permeability & $k_{h}=10^{-13} \mathrm{~m}^{2}, k_{v}=10^{-14} \mathrm{~m}^{2}$ \\
Porosity & $\Phi=0.12$ \\
Pore Compressibilty & $c=4.5 \times 10^{-10} \mathrm{~Pa}^{-1}$ \\
Aquifer thickness & $400 \mathrm{~m}$ \\
Diffusivities for all components & in gas phase: $D=1.0 \times 10^{-6} \mathrm{~m}^{2} / \mathrm{s}$ \\
\hline Relative Permeability & in aqueous phase: $D=1.0 \times 10^{-9} \mathrm{~m}^{2} / \mathrm{s}$ \\
liquid: van Genuchten $[1980]$ & \\
$k_{r l}=\sqrt{S^{*}}\left[1-\left(1-\left[S^{*}\right]^{1 / m}\right)^{m}\right]^{2}$ & $S^{*}=\left(S_{l}-S_{l r}\right) /\left(1-S_{l r}\right)$ \\
irreducible water saturation & $S_{l r}=0.30$ \\
exponent & $m=0.457$ \\
gas: Corey $[1954]$ & $\hat{S}^{2}=\frac{\left(S_{l}-S_{r}\right)}{\left(1-S_{l r}-S_{g r}\right)}$ \\
$k_{r g}=(1-\hat{S})^{2}\left(1-\hat{S}^{2}\right)$ & $S_{g r}=0.05$ \\
irreducible gas saturation &
\end{tabular}

Capillary pressure

van Genuchten function

$P_{\text {cap }}=-P_{0}\left(\left[S^{*}\right]^{-1 / m}-1\right)^{1-m} \quad S^{*}=\left(S_{l}-S_{l r}\right) /\left(1-S_{l r}\right)$

irreducible water saturation $\quad S_{l r}=0.0$

exponent $\quad m=0.457$

strength coefficient $\quad P_{0}=19.61 \mathrm{kPa}$

Initial Conditions

Salinity

3.2 wt.- $\%$

Pressure

180 bar

Temperature

$50{ }^{\circ} \mathrm{C}$ 
instability occurs

$$
t_{c}=\frac{c_{1}(\mu \phi)^{2} D}{\left(g \Delta \rho k_{h}\right)^{2}}
$$

where $c_{1}$ is a numerical constant in the range of 80-100. Ennis-King and Paterson [2003] also proposed that the critical wavelength $\lambda_{c}$ of the instability can be approximated by:

$$
\lambda_{c}=\frac{c_{2} \mu \phi D}{g \Delta \rho k_{h}}
$$

where $c_{2}$ is again a numerical constant in the range of 100-120. Using the parameters in Table 4.3, Equations 4.4 and 4.5 give values for $t_{c} \sim 1$ year and $\lambda_{c} \simeq 6.7$ meters. This preliminary calculation shows that the numerical results presented in Figure 4.15 reveal fingers at later time and with larger widths. Therefore, a sufficiently refined grid is required refinement to properly represent the finger structure. Finally, Figure 4.16 shows that convective mixing considerably enhances the dissolution process. 

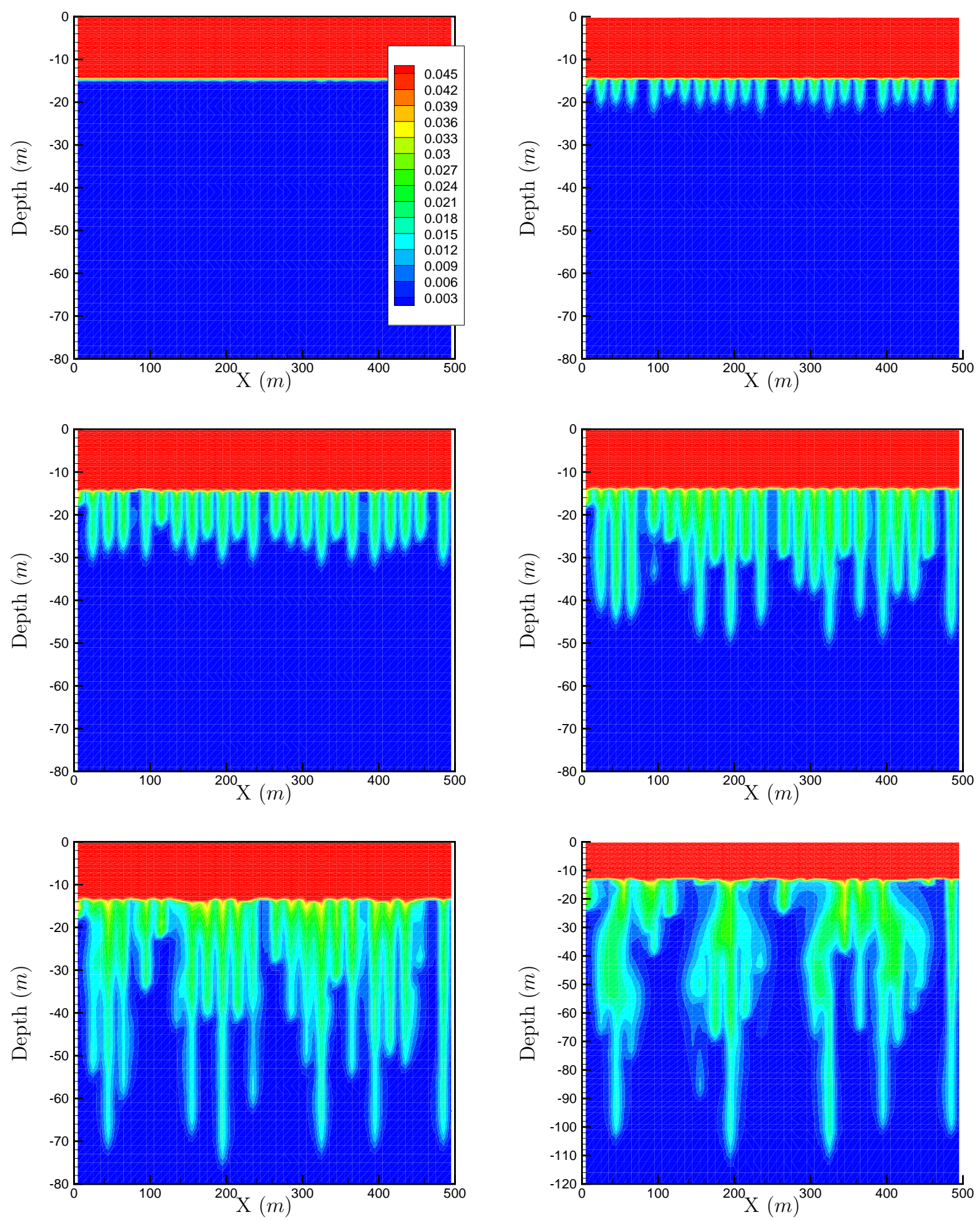

Figure 4.15: Dissolved $\mathrm{CO}_{2}$ in the aqueous phase contours at $t=100,300,500,800$, 1200 , and 1800 years 


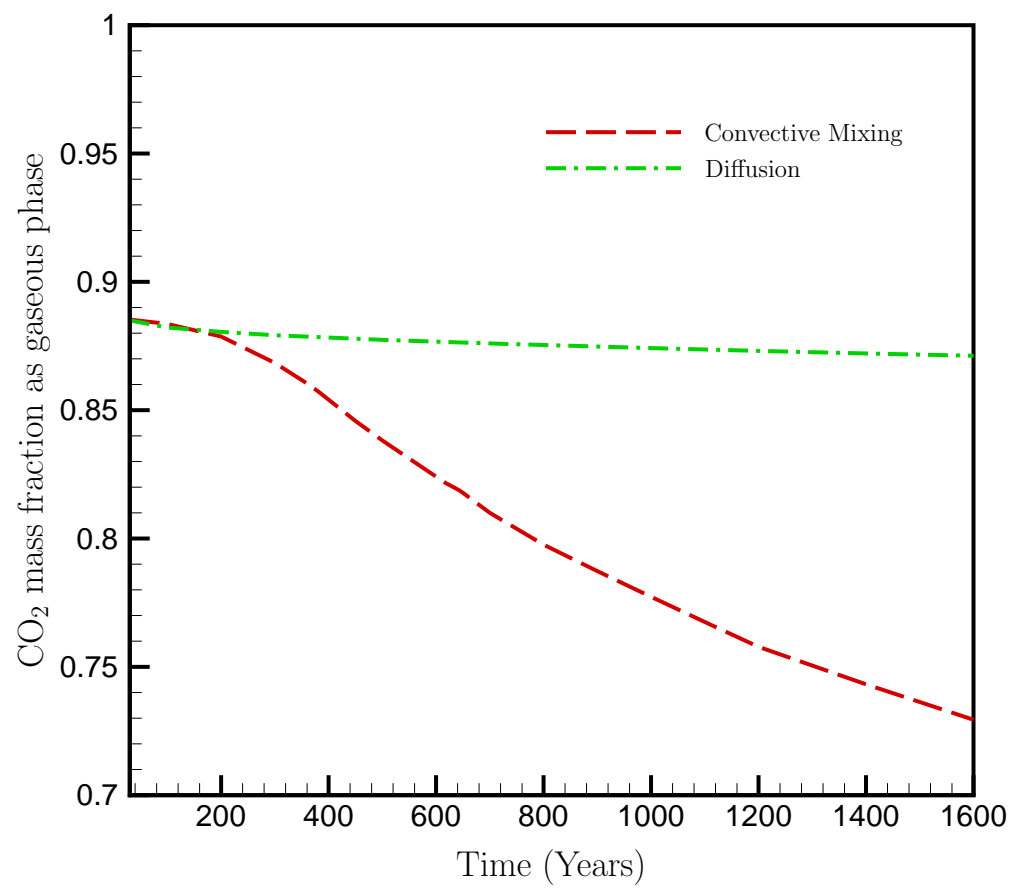

Figure 4.16: Simulated mass fraction ratio as free gaseous phase. Diffusion curve is obtained from numerical simulations that ignore the effect of $\mathrm{CO}_{2}$ content in aqueous phase density. 


\section{Chapter 5}

\section{Fluid dynamic instabilities}

\subsection{Introduction}

The process of carbon dioxide $\left(\mathrm{CO}_{2}\right)$ injection into saline aquifers can be classified as immiscible displacement of an aqueous phase by a less dense and less viscous gas phase. Under disposal conditions (supercritical $\mathrm{CO}_{2}$ ) the viscosity of carbon dioxide can be less than the viscosity of the aqueous phase by a factor of 15 . Because of the lower viscosity, the $\mathrm{CO}_{2}$ displacement front will have a tendency towards instability so that waves or rounded lobes of saturation may appear and grow into fingers that lead to enhanced dissolution, bypassing, and possibly poor sweep efficiency. Mechanisms of viscous fingering and fluid displacements in porous media have been experimentally described by the early work of Hill [1952], van Meurs [1957], Saffman and Taylor [1958] and Chuoke et al. [1959].

Viscous fingering has been extensively studied for the case of miscible displacement. Sahimi [1993] and Homsy [1987] provided excellent reviews of the theory of viscous fingering in porous media. Zimmerman and Homsy [1992] studied the effects of viscosity contrast and dispersion in three-dimensional finger propagation. Tchelepi and Orr [1994] addressed issues of viscous fingering with high-resolution 
two- and three-dimensional simulations of flow in homogeneous and heterogeneous porous media. More recently, De Wit and Homsy [1999a,b] studied viscous fingering in reaction-diffusion systems. Pankiewitz and Meiburg [1999], Ruith and Meiburg [2000] and Camhi et al. [2000] performed high resolution numerical simulations of miscible displacements with gravity override. On the other hand only a few previous numerical studies have examined viscous fingering due to immiscible displacement in porous media. Christie [1989] and Blunt et al. [1992] performed a series of viscous fingering simulations for compositional and immmiscible flows to investigate the effect of instabilities on oil recovery.

Figure 5.1 shows the calculated $\mathrm{CO}_{2}$ viscosity profile and the corresponding viscosity ratio $\left(\mu_{\mathrm{H}_{2} \mathrm{O}} / \mu_{\mathrm{CO}_{2}}\right)$ up to a depth of $2000 \mathrm{~m}$. For this calculation a temperature gradient of $3^{\circ} \mathrm{C} / 100 \mathrm{~m}$ and surface temperature of $10^{\circ} \mathrm{C}$ was assumed. Below 800 $\mathrm{m}$, approximated minimum depth required to sustain $\mathrm{CO}_{2}$ supercritical conditions, the viscosity contrast between carbon dioxide and water is moderate with a viscosity ratio ranging from 22 at $800 \mathrm{~m}$, to 10 at $2000 \mathrm{~m}$.

Results of high-resolution numerical simulations of the onset of instabilities (viscous fingering) during injection of $\mathrm{CO}_{2}$ into saline aquifers are presented in this chapter. Specifically, the influence of viscosity ratio, relative permeability functions, and capillary pressure on finger growth and spacing is explored. In addition, the issues of finger triggering, convergence under grid refinement and boundary condition effects are considered also. 


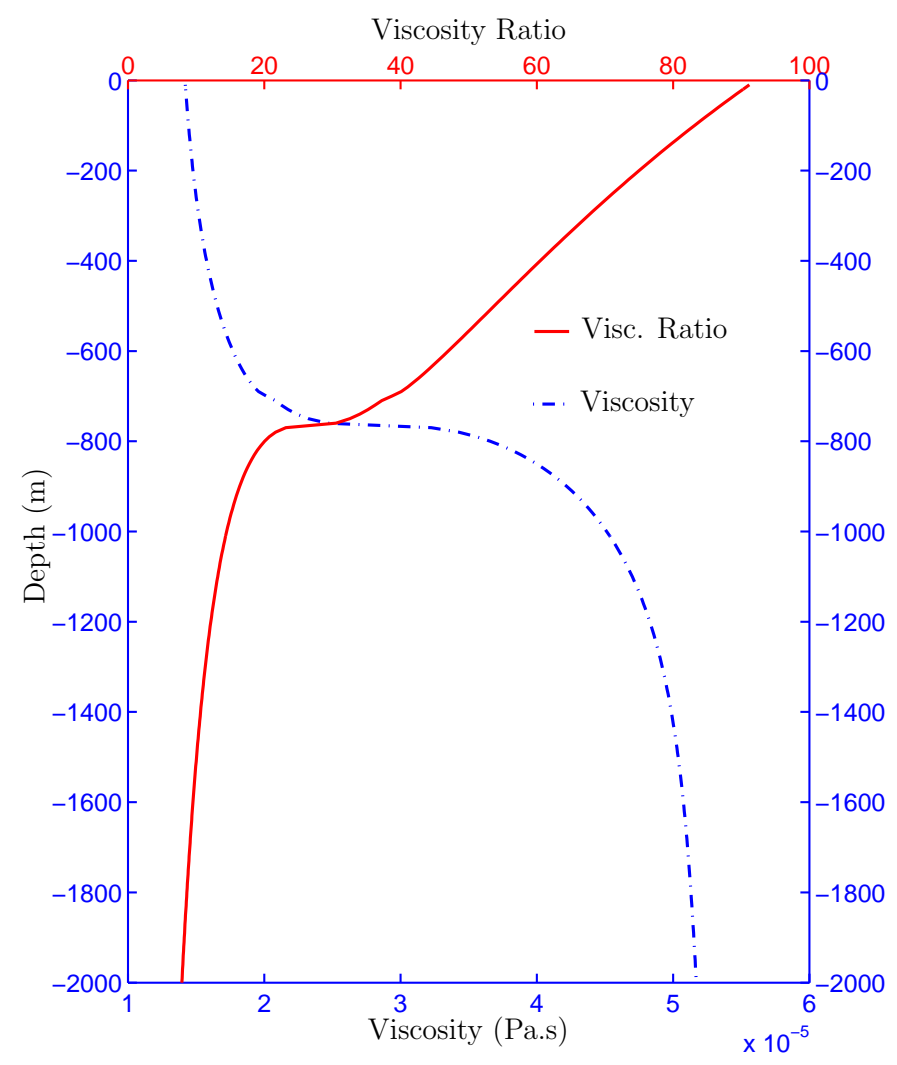

Figure 5.1: $\mathrm{CO}_{2}$ viscositiy and viscosity ratio $\frac{\mu_{\mathrm{H}_{2} \mathrm{O}}}{\mu_{\mathrm{CO}_{2}}}$ profile.

\subsection{Mechanisms of Viscous Fingering}

For illustration purposes consider a rectilinear fluid displacement where a fluid of viscosity $\mu_{2}$ and density $\rho_{2}$ is displacing a fluid of viscosity $\mu_{1}$ and density $\rho_{1}$ (Figure 5.2). Darcy's law holds for each fluid phase separately:

$$
\frac{d p}{d x}=-\mu U / k+\rho g
$$

Now consider a sharp interface or zone where density and viscosity all change rapidly, e.g. a zone such as that shown in Figure 5.2. Suppose that a small perturbation C-D, of depth $\delta x$, develops in the interface A-B. Let $P_{0}$ be the pressure at the 
level A-B, $P_{1}$ the pressure just above $\mathrm{C}-\mathrm{D}$ and $P_{2}$ the pressure just below $\mathrm{C}-\mathrm{D}$. The depression will disappear if $P_{1}>P_{2}$, and will increase if $P_{2}>P_{1}$, with $P_{1}$ and $P_{2}$ given by:

$$
\begin{aligned}
& P_{1}=P_{0}+\rho_{1} g \delta x-v \frac{\mu_{1}}{k_{1}} \delta x \\
& P_{2}=P_{0}+\rho_{2} g \delta x-v \frac{\mu_{2}}{k_{2}} \delta x
\end{aligned}
$$

where $k_{1}$ and $k_{2}$ are the effective permeabilities and $v$ is the Darcy velocity of the interface. Therefore, the condition for unstable flow is:

$$
P_{2}-P_{1}=g\left(\rho_{2}-\rho_{1}\right) \delta x-v\left(\frac{\mu_{2}}{k_{2}}-\frac{\mu_{1}}{k_{1}}\right) \delta x>0
$$

leading to an expression for the critical Darcy velocity $\left(v_{\text {crit }}\right)$ :

$$
v_{c r i t}>\frac{g\left(\rho_{1}-\rho_{2}\right)}{\left(\frac{\mu_{1}}{k_{1}}-\frac{\mu_{2}}{k_{2}}\right)}
$$

The above simplified analysis was first presented by Hill [1952] for the simple case of quasi two-dimensional miscible fluid displacement where $k_{1}=k_{2}=k$. If the displacement velocity exceeds the critical value, the perturbation will be amplified, leading to development of fingers. Consider now the case where $\mathrm{CO}_{2}$ displaces $\mathrm{H}_{2} \mathrm{O}$. For $\rho_{\mathrm{CO}_{2}}>\rho_{\mathrm{H}_{2} \mathrm{O}}$ and $\mu_{\mathrm{CO}_{2}}<\mu_{\mathrm{H}_{2} \mathrm{O}}$ the critical velocity is less than zero, so that the system is unstable even when $v=0$. Both gravity and viscosity tend to destabilize the interface. This extreme case can only be possible at higher pressure and low temperatures that are not encountered in aquifer disposal conditions. Under disposal conditions $\mu_{\mathrm{CO}_{2}}<\mu_{\mathrm{H}_{2} \mathrm{O}}$ and $\rho_{\mathrm{CO}_{2}}<\rho_{\mathrm{H}_{2} \mathrm{O}}$. Thus, gravity is a stabilizing force, while viscosity is destabilizing. 


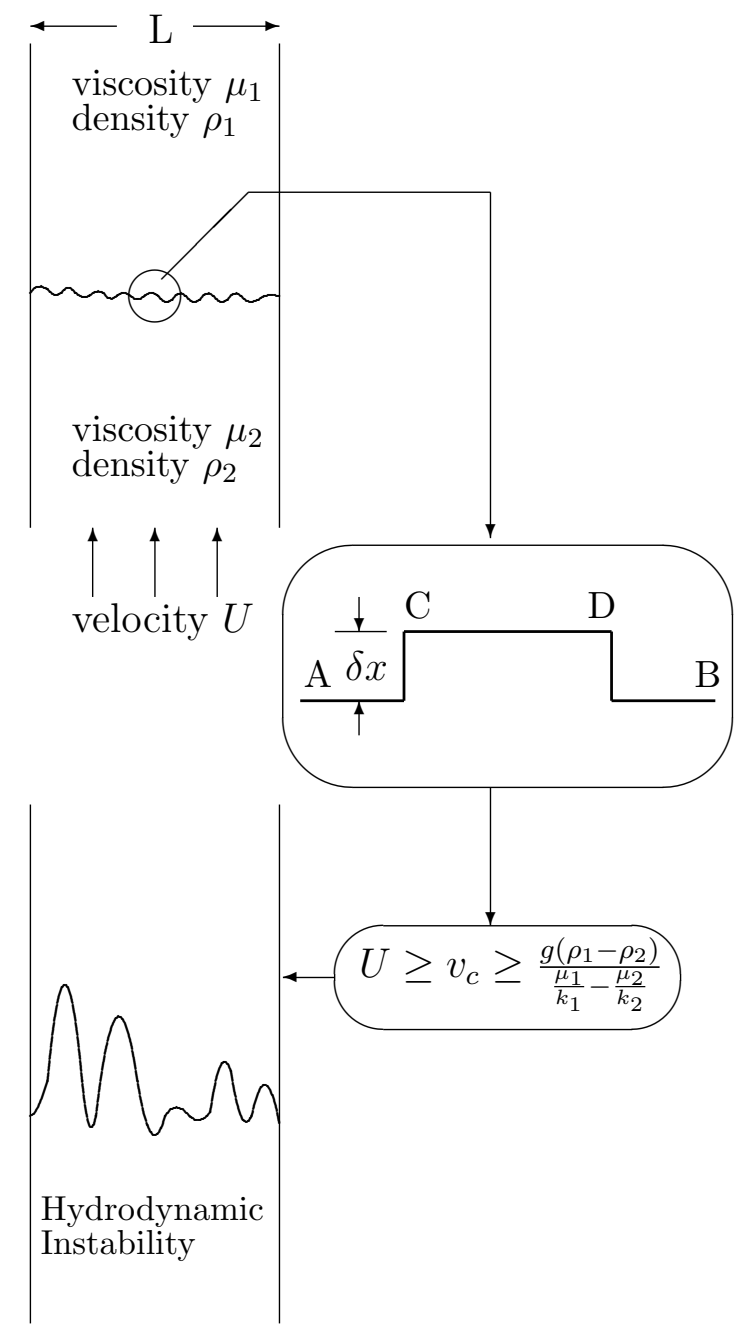

Figure 5.2: Viscous fingering in rectilinear flow

\subsection{Problem Formulation}

A description of the base problem used to study the possible outcome of viscous fingering is presented in this section. The analysis is based on two-phase flow in two-dimensional, isotropic systems that imply the absence of gravity override.

\subsubsection{Governing Equations}

In this formulation we assume, (1) zero mass transfer between fluid phases, (2) negligible rock compressibility, (3) immiscible flow, (4) incompressible fluids, (5) zero 
sources and sinks, (6) multiphase flow extension of Darcy's Law and (7) all the dependent variables are volumetric averages over a representative elementary volume (REV) [Bear, 1972]. Less restrictive assumptions will be made in the numerical simulations below. Under assumption (1), the mass balance equation for each fluid phase $\alpha$ may be written as

$$
\frac{\partial}{\partial t}\left(\phi \rho_{\alpha} S_{\alpha}\right)+\nabla\left(\mathbf{f}_{\alpha}\right)=0
$$

where $\rho_{\alpha}$ is the density of phase $\alpha, S_{\alpha}$ is the saturation of phase $\alpha$ and $\phi$ is the porosity, with $\alpha \equiv g$ for gas and $\alpha \equiv l$ for liquid; $\mathbf{f}_{\alpha}$ is the flux of phase $\alpha$. The flux of each phase, $\mathbf{f}_{\alpha}$, is related to the pressure gradient in terms of an extension of Darcy's law.

$$
\mathbf{f}_{\alpha}=\rho_{\alpha} \boldsymbol{v}_{\alpha}=-\rho_{\alpha} \frac{\mathbf{k} k_{r \alpha}}{\mu_{\alpha}}\left(\nabla P_{\alpha}-\rho_{\alpha} \mathbf{g}\right)
$$

where $\boldsymbol{v}_{\alpha}$ is the volumetric flux of phase $\alpha, \mathbf{k}$ is the intrinsic permeability tensor, $k_{r \alpha}$ is the relative permeability of phase $\alpha$, and $\mathbf{g}$ is the gravitational vector (the vertical coordinate is oriented positive downward). The pressures of the two phases are related through the capillary pressure, $P_{c}=P_{g}-P_{l}$. Relative permeability functions and capillary pressure are assumed to be functions of phase saturations. Equation 5.6 is subject to the constraint that the two fluids jointly fill the void space, $S_{g}+S_{l}=1$.

\subsubsection{Flow system}

The flow system under consideration (Figure 5.3) consists of a two dimensional porous medium domain with a constant cross-sectional area. The porous medium is considered homogeneous and isotropic and the effects of gravity are neglected. The 
system is initially at uniform liquid saturation $S_{l}$ and gas is injected along the left boundary. The top and bottom boundaries are initially assumed no flow boundaries, and the domain is considered sufficiently long in the $\mathrm{x}$ direction so that the system would be infinite-acting for the time periods simulated.

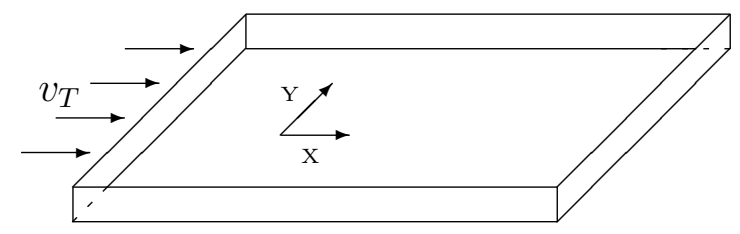

Figure 5.3: Schematic of flow system.

The governing flow equations 5.6 and 5.7 are a set of coupled, nonlinear partial differential equations. These basic equations can be mathematically manipulated into several alternate forms with choices of primary dependent variables [Binning and Celia, 1999]. For simplicity, consider the unidirectional flow with constant porosity and fluid densities. Then the flow is described by the gas saturation equation:

$$
\phi \frac{\partial S_{g}}{\partial t}+v_{T} \frac{\partial f_{g}}{\partial x}=+\frac{\partial}{\partial x}\left[\lambda_{l} f_{g} \frac{d P_{c}}{d S_{g}} \frac{\partial S_{g}}{\partial x}\right]
$$

where $v_{T}=v_{g}+v_{l}$ is the total Darcy velocity (volumetric flux),

$$
f_{g}=\frac{\lambda_{g}}{\lambda_{T}}
$$

is the fractional flow of the gas phase [Buckley and Leverett, 1942],

$$
\lambda_{\alpha}=\frac{k_{r \alpha}}{\mu_{\alpha}}
$$


is the relative mobility of phase $\alpha$, and $\lambda_{T}=\lambda_{g}+\lambda_{l}$ is the total mobility. In the absence of flow perturbations, a steady one-dimensional (1D) solutions develops moving along the direction of displacement, $x$, with a constant velocity, $v$. The velocity and shape of this front is dictated by the variation of relative permeabilities and capillary pressure as a function of saturation. For the typical displacement where capillary effects are assumed negligible, the saturation equation reduces to the Buckley-Leverett equation which may be solved analytically.

$$
\phi \frac{\partial S_{g}}{\partial t}+v_{T} \frac{\partial f_{g}}{\partial x}=0
$$

The Buckley-Leverett solution gives a saturation profile with a sharp front along the flow direction. When the initial saturation is uniform, the graphical approach developed by Welge [1952] can be used to determine the saturation front [Wu et al., 1990].

\subsection{3 $\mathrm{CO}_{2}-\mathrm{H}_{2} \mathrm{O}$ case}

As an example, the relative permeability functional forms determined by van Genuchten [1980] and Corey [1954] are used to derive the fractional flow function and the Buckley-Leverett Solution.

$$
\begin{gathered}
k_{r l}=\sqrt{S^{*}}\left\{1-\left(1-\left[S^{*}\right]^{1 / \lambda}\right)^{\lambda}\right\}^{2} \\
S^{*}=\frac{S_{l}-S_{l r}}{1-S_{l r}} \\
k_{r g}=(1-\hat{S})^{2}\left(1-\hat{S}^{2}\right)
\end{gathered}
$$




$$
\hat{S}=\frac{S_{l}-S_{l r}}{1-S_{l r}-S_{g r}}
$$

For $\mathrm{CO}_{2}$ and water, $\mu_{g}=4.0 \cdot 10^{-5} \mathrm{~N} \mathrm{~s} \mathrm{~m}^{-2}$ and $\mu_{l}=7.5 \cdot 10^{-4} \mathrm{~N} \mathrm{~s} \mathrm{~m}^{-2}$ at $(T, P)=\left(82 \mathrm{bar}, 33{ }^{\circ} \mathrm{C}\right)$, and relative permeability parameters $\lambda=0.85, S_{l r}=0.25$, and $S_{g r}=0.05$ we obtain the fractional flow curve $f_{g}$ shown in Figure 5.4. Also shown in the figure is the tangent that originates at the initial gas saturation. The point of tangency defines the gas saturation at the displacement front and the slope of the Welge tangent gives the speed of the shock.

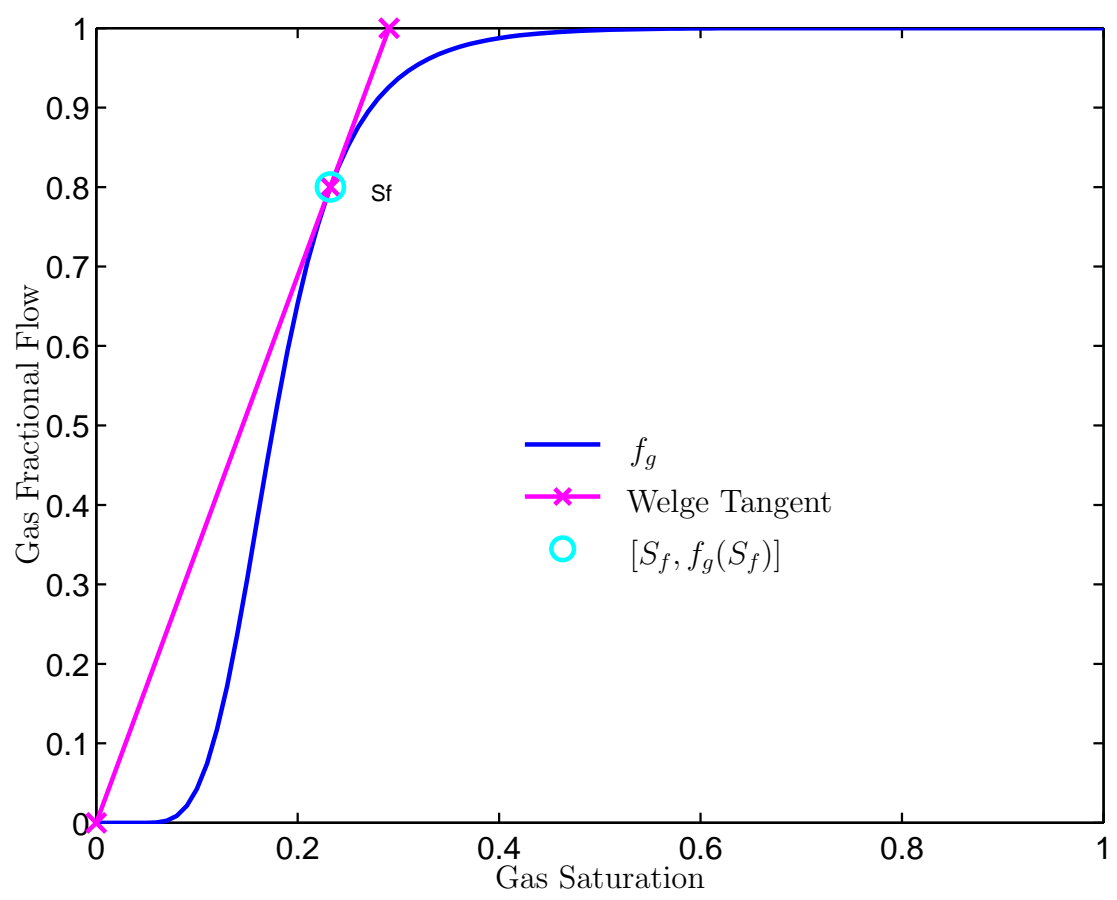

Figure 5.4: Fractional Flow Function.

Figures 5.5 and 5.6 show the total mobility and the Buckley-Leverett solution together with a numerical solution obtained with a front-tracking version EOS3f of TOUGH2's module EOS3 [Pruess et al., 1999]. On the continuum level, the severity of the 


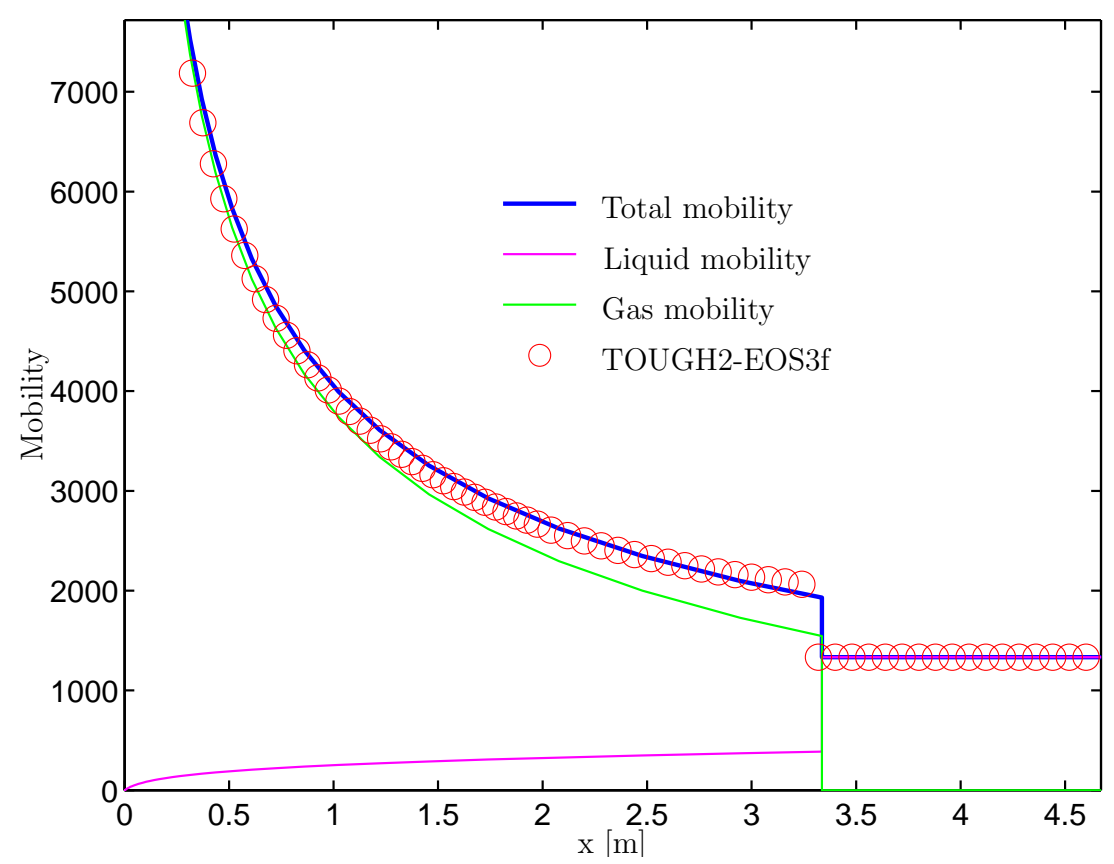

Figure 5.5: Buckley-Leverett solution for mobilities. Symbols represent the TOUGH2-EOS3f solution.

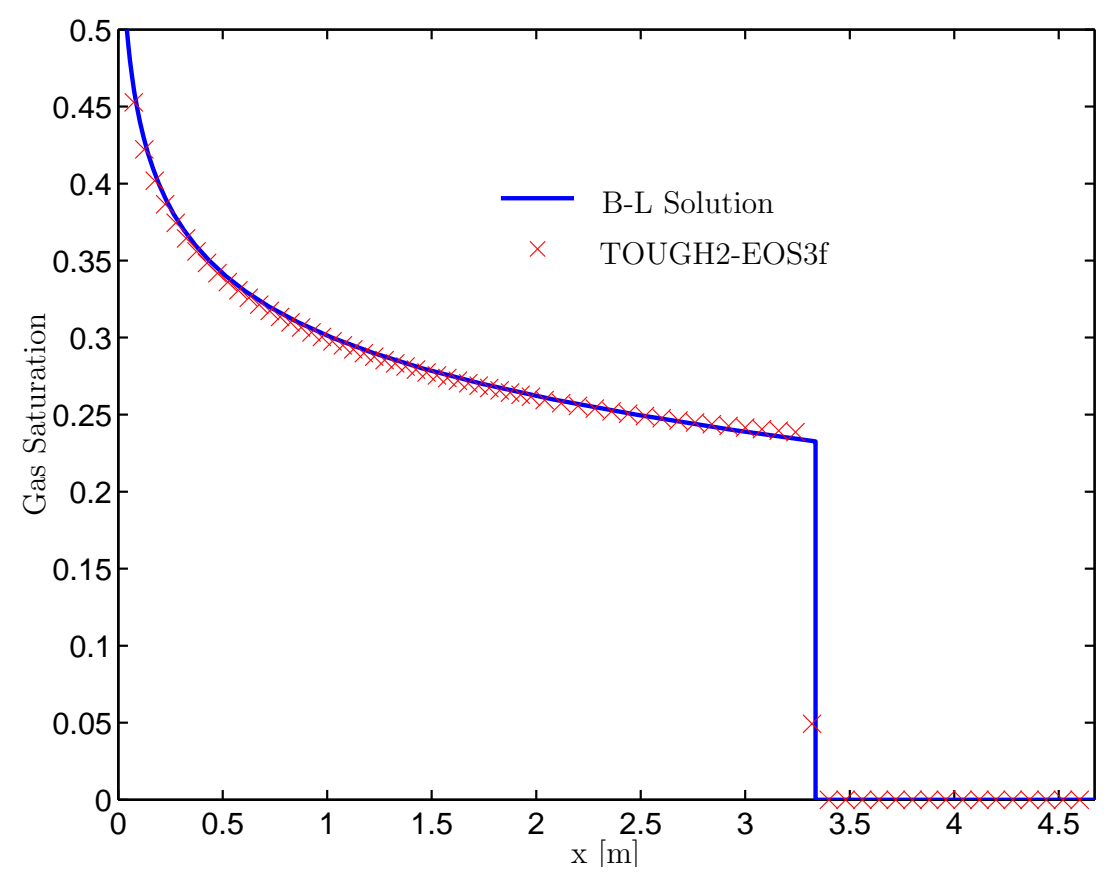

Figure 5.6: Buckley-Leverett solution for gas saturation. Symbols represent the numerical solution with front tracking (TOUGH2-EOS3f). 
instability is controlled by the ratio of the total mobilities upstream and downstream of the front:

$$
\lambda_{\text {shock }}=\frac{\lambda_{T}^{u p}}{\lambda_{T}^{\text {down }}}
$$

where upstream and downstream shock saturation and mobility values are denoted by $S_{g}^{u p}$ and $S_{g}^{\text {down }}, \lambda_{T}^{u p}$ and $\lambda_{T}^{\text {down }}$. Instabilities may occur when $\lambda_{\text {shock }}>1$ while displacements whith $\lambda_{\text {shock }} \leq 1$ will proceed stably.

The general view is that because the viscosity ratio $\mu_{\mathrm{H}_{2} \mathrm{O}} / \mu_{\mathrm{CO}_{2}}$ is larger than unity, hydrodynamic unstable displacement will occur, no matter the injection conditions. The previous misleading assertion is often based on a large amount of immiscible flow observations in Hele-Shaw cells, and on past porous media investigations based on the assumption that single-phase flow regions are separated by an abrupt macroscopic interface. The approximation that only one phase flows upstream of the front, and only the other flows downstream is not valid for real porous materials. Immiscible displacement in porous media is characterized by simultaneous flow of both phases. In this case, it is the total mobility ratio, the sum of the mobilities of the two phases behind the front divided by the sum ahead that dictates the frontal instability: ratios larger than unity indicate instability and less than unity, stability. Thus, it is not only the viscosity ratio that is crucial to assert the stability but its interplay with the relative permeability functions through the fluid mobilities.

Figure 5.7 shows the variation of mobility shock $\left(\lambda_{\text {shock }}\right)$, evaluated on the basis of a Buckley-Leverett shock front, with viscosity ratio. For illustration purposes we considered quadratic and cubic relative permeability functions as well as those given by Equations 5.12 and 5.14 with $S_{l r}=0.25$ and $S_{g r}=0.05$. Mobility ratios are seem to be substantially smaller than viscosity ratios, indicating that flow instabilities in porous media will be weaker than for fluid displacements in "free" space. For $\mathrm{CO}_{2}$ and 
$\mathrm{H}_{2} \mathrm{O}$ (viscosity ratios between 10 and 100) the use of quadratic and cubic equations predicts always unstable displacements. Instability when using van Genuchten [1980] in conjunction with Corey [1954] relative permeabilities depend on the shape factor $\lambda$. For $\lambda$ values lower than 0.6 , we expect stable displacements.

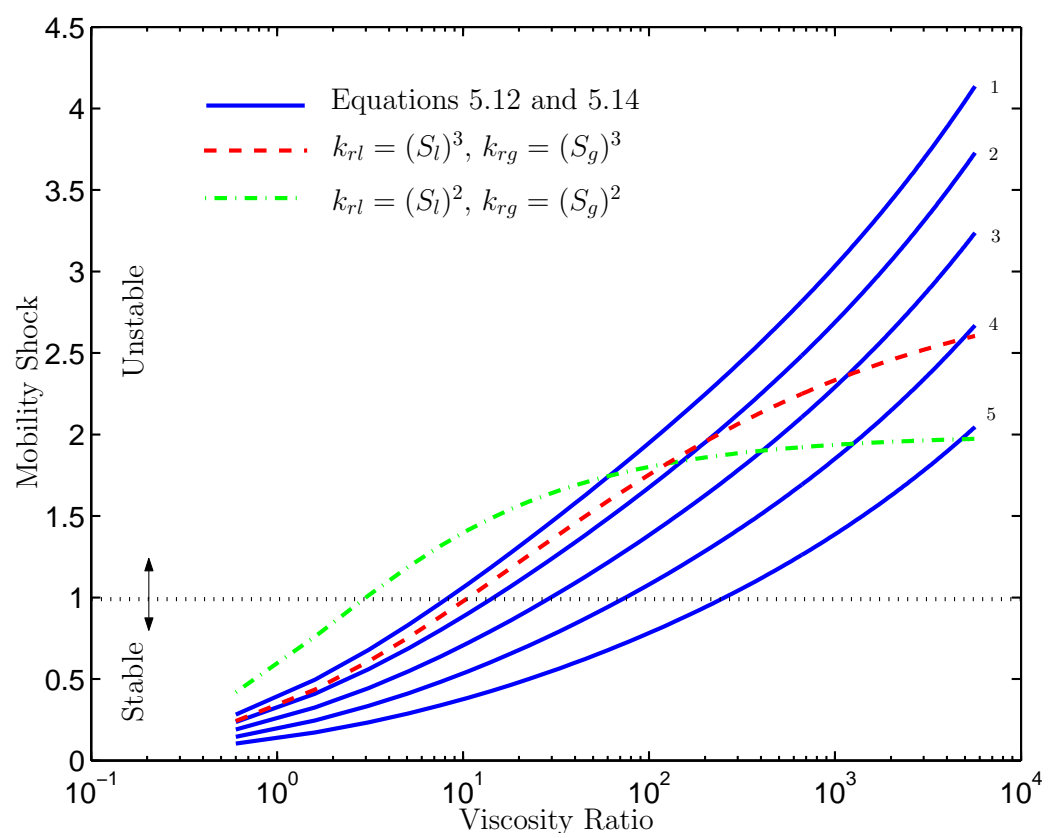

Figure 5.7: Stability of immiscible displacement for different relative permeability functions. Curves $1,2,3,4$, and 5 refer to values of $\mathrm{m}=0.9,0.8,0.7,0.6,0.5$ respectively in the van Genuchten [1980] relative permeability function.

Figure 5.8 shows calculated mobility shock profiles obtained from the BuckleyLeverett solution using the viscosity ratio profile shown in Figure 5.1. For this calculation, we considered the relative permeability functions described above with shape factors $\lambda=0.7,0.8$ and 0.9 for equations 5.12 and 5.14. For $\lambda=0.7$, we expect stable displacements at all depths. When $\lambda=0.8$ or $\lambda=0.9$, instability would occur above $1000 \mathrm{~m}$ or $1900 \mathrm{~m}$, respectively. The use of quadratic equations predicts always unstable displacements, whereas the use of cubic equations predicts unstable displacements above $1400 \mathrm{~m}$. 

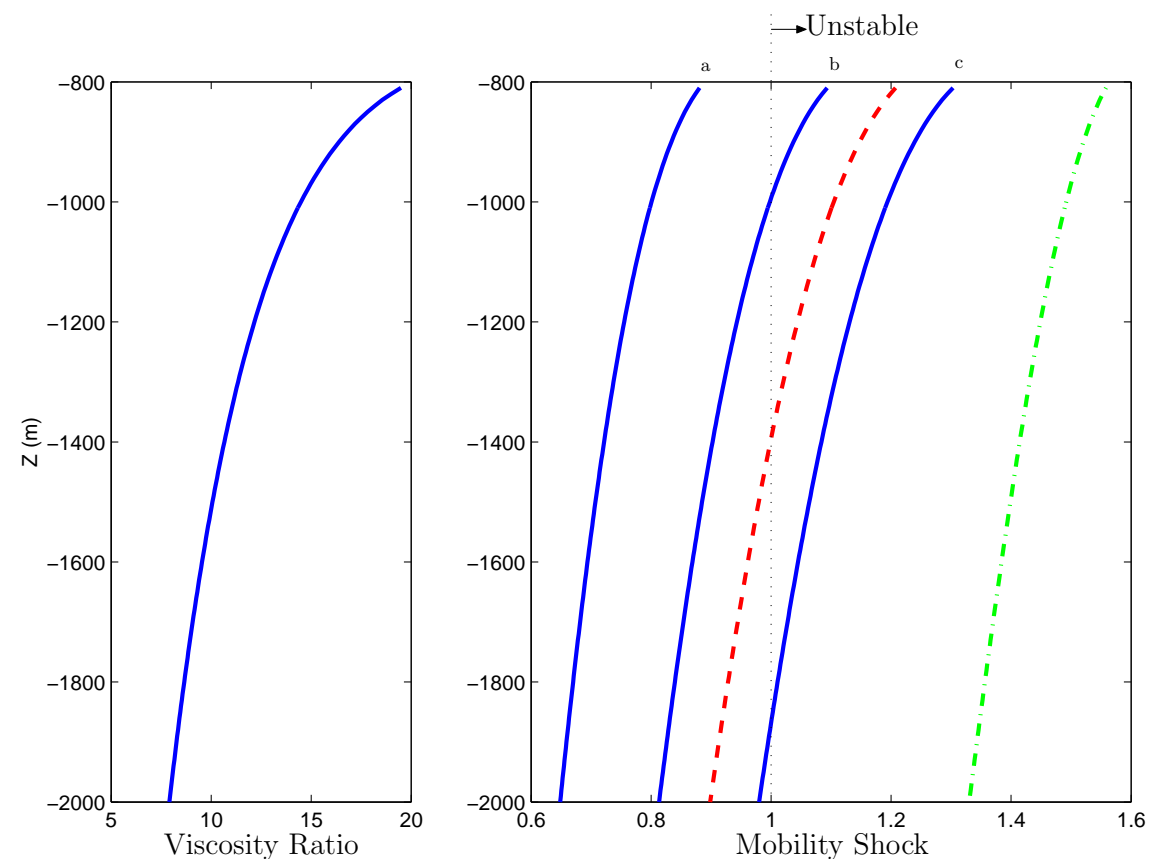

Figure 5.8: Mobility shock profile for different relative permeability functions. Curves $\mathrm{a}, \mathrm{b}$, and $\mathrm{c}$, refer to values of $\lambda=0.7,0.8$ and 0.9 respectively in the van Genuchten [1980] relative permeability function. 


\subsection{Numerical implementation}

Viscous fingering was simulated using the TOUGH2 modules ECO2 (two phase flow of brine and gas with three mass components, water, salt and carbon dioxide, Chapter 3) and EOS3 (two-phase flow of water and air, [Pruess et al., 1999]). Minor changes to EOS3 module were necessary in order to incorporate a set of fluid properties for different gas and liquid phases. A summary of the changes and a description of the computer platforms used is presented in Table 5.1.

When using EOS3 module, $\mathrm{CO}_{2}$ is treated as an air-like gas as we do not attempt to capture all the fluid flow dynamics features due to the injection of $\mathrm{CO}_{2}$ into water. Instead, our intent is to explore the possible outcome of instabilities when a fluid with $\mathrm{CO}_{2}$ viscosity displaces water, and this can be accomplished with the modified version of EOS3.

\subsubsection{Methods of triggering fingering}

Several approaches can be used to trigger viscous fingers. Truncation and roundoff errors seem to be the most tempting approach to perturb the displacement front and for that purpose one can reduce the convergence tolerance. Although successful in triggering fingers, this approach is not recommended as it does not allow to ascertain convergence under grid refinement. In our study we trigger fingers by one of two methods:

1. A random permeability field.

2. A finite amplitude perturbation of the inlet conditions during the first time step. 


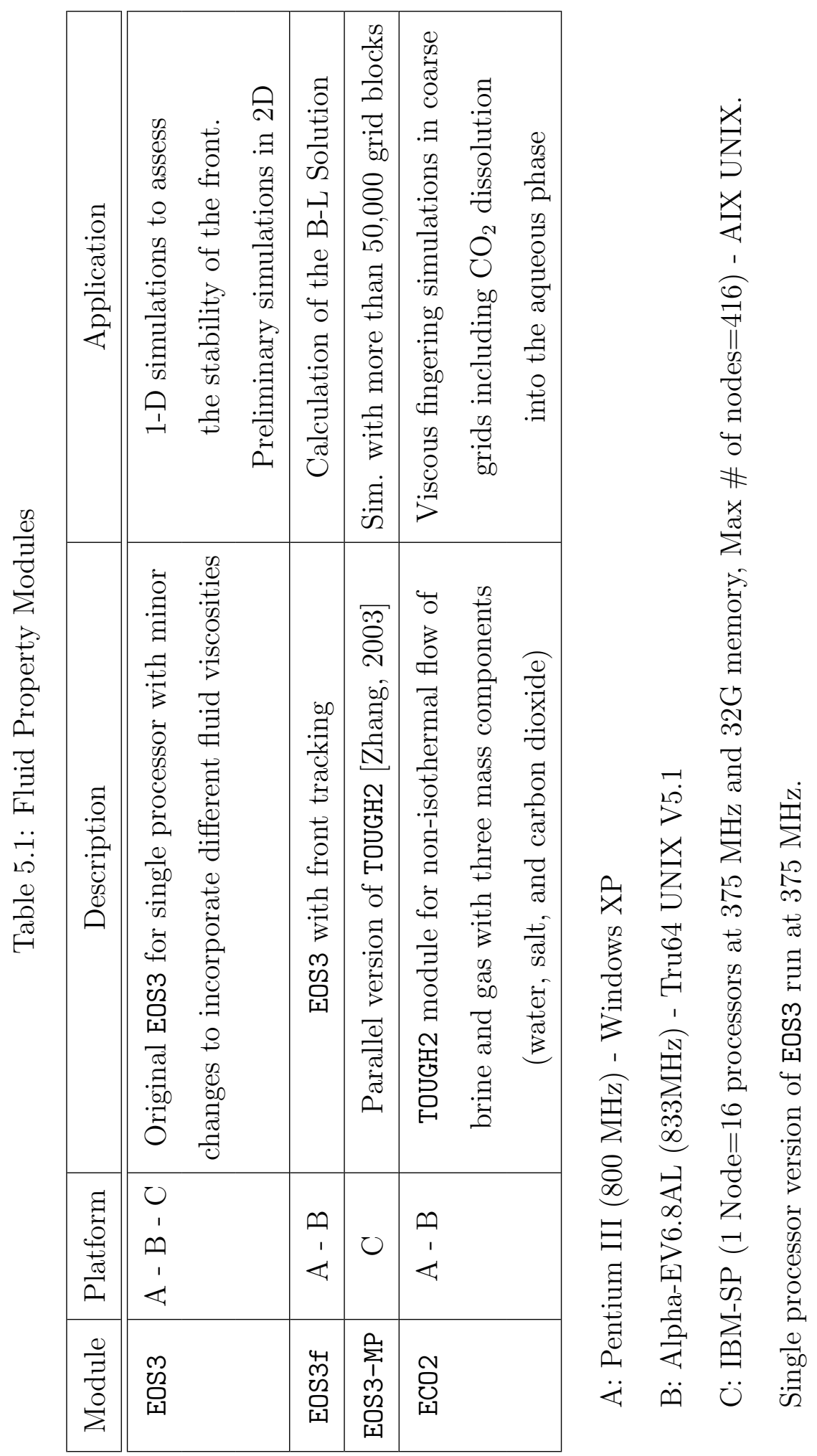


A mild random modifications of the permeability field, usually in the order of 4

$\%\left(0.96 \times \mathbf{k} \leq \mathbf{k}_{\text {mod }} \leq 1.04 \times \mathbf{k}\right)$, was made using the permeability modification capabilities already incorporated in TOUGH2. The simulations show that the same finger structure is preserved whether the entire domain is perturbed or the permeability modification is localized in a strip after the injection boundary. Figure 5.9 shows that for a mobility shock, $\lambda_{\text {shock }}=3.25$ fingers are triggered when the permeability is modified; the displacement is uniform otherwise.

\subsubsection{Convergence under grid refinement}

Simulation results show that finger growth and finger wave length are strongly affected by grid size. When the capillary diffusion effect is ignored in the gas saturation Equation 5.8, short wave length fingers are damped by the mesh size, and the solution obtained depends on the grid size no matter how much the grid is refined. When capillary effects are considered, it is possible to obtain solutions that converge under grid refinement. Our simulations show that for some cases an extremely fine grid (more than 100,000 grid blocks) is required (Figure 5.10). This requires simulation with the massive parallel scheme developed by Zhang [2003] and Wu et al. [2002].

Figure 5.11 shows viscous fingering predictions obtained from a relative coarsegrid calculation on a scalar machine (Alpha-EV6.8AL, Table 5.1) and from a highresolution simulation using a massively parallel scheme (IBM-SP, Table 5.1). The fine grid results, or "converged" solution, show finger widths of $1 \mathrm{dm}$. A similar finger pattern is observed for coarse-grid simulations, demonstrating that calculations on scalar machines could be used for approximate finger predictions. 
(a)

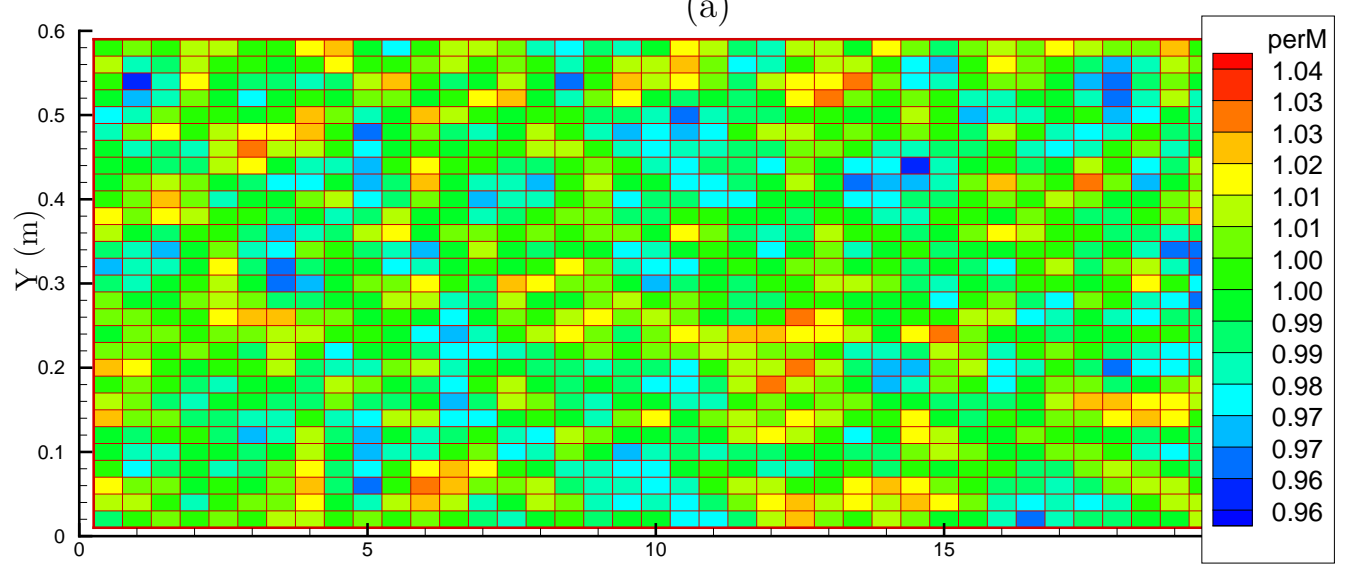

(b)

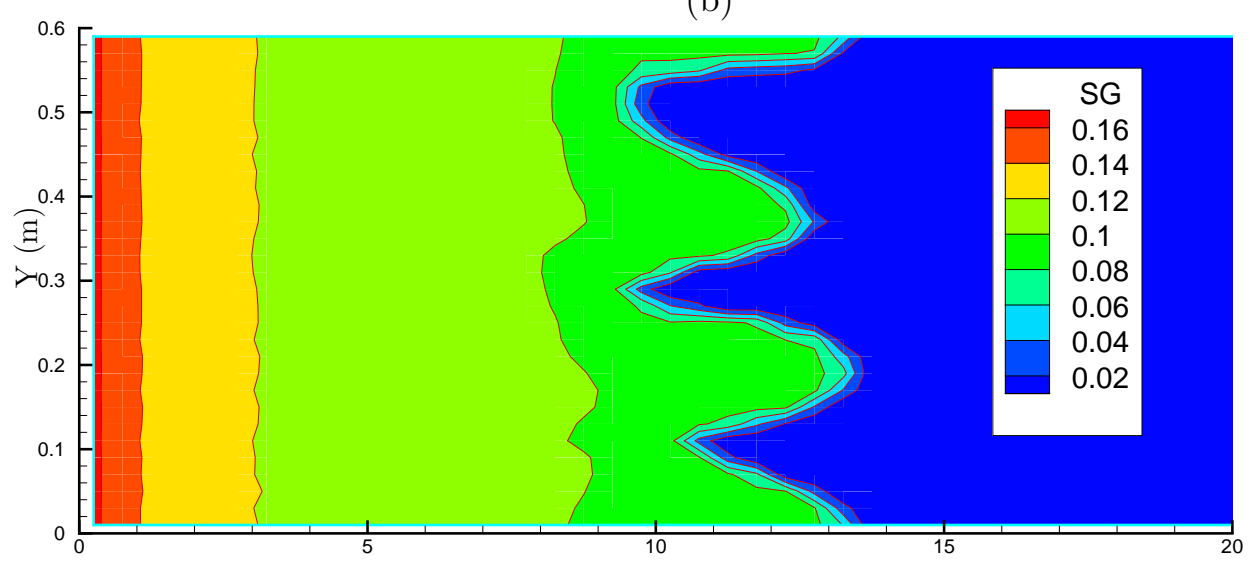

(c)

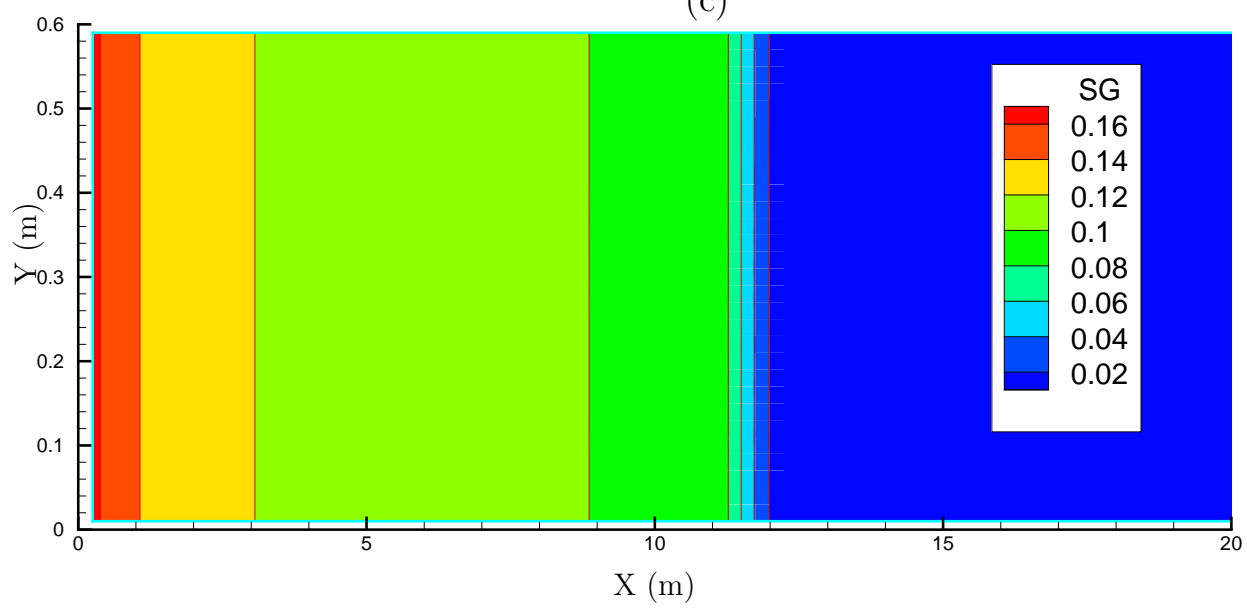

Figure 5.9: Viscous fingers are triggered (b) when a modified permeability field is used (a). No fingering occurs otherwise (c). 
(a)

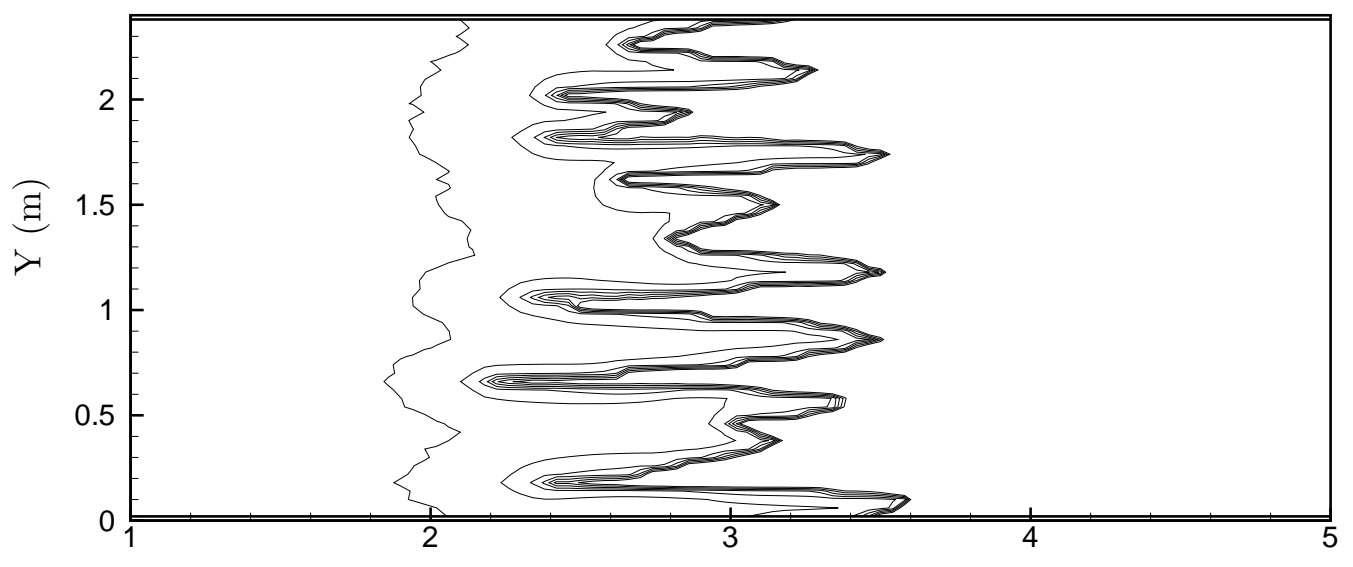

(b)

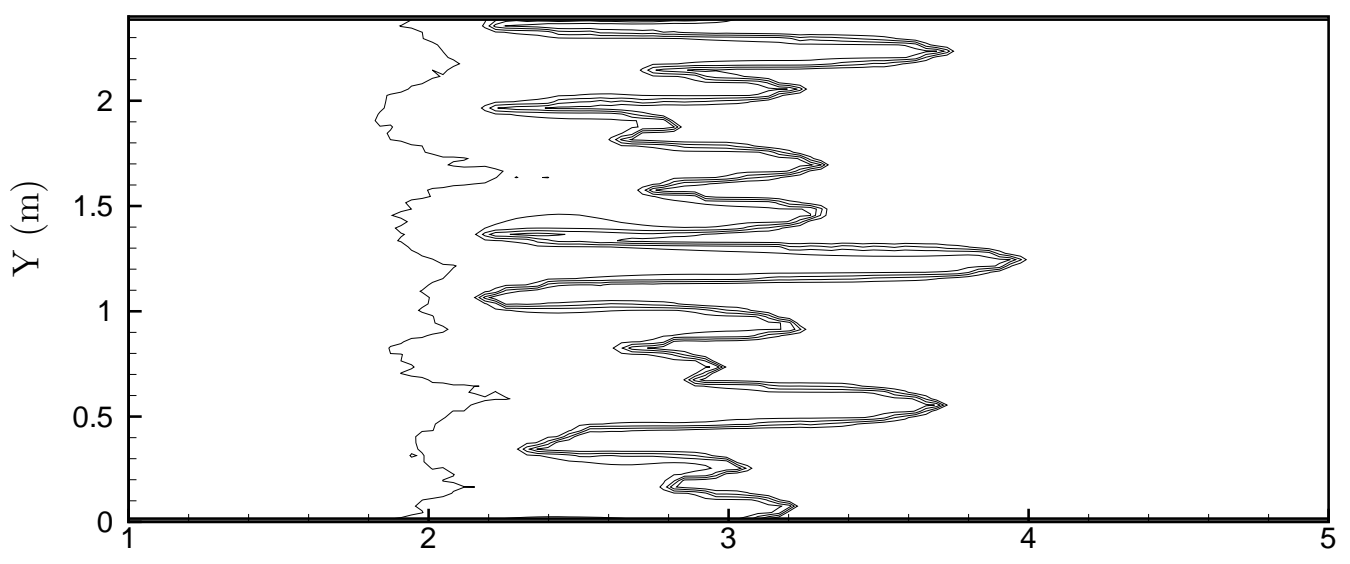

(c)

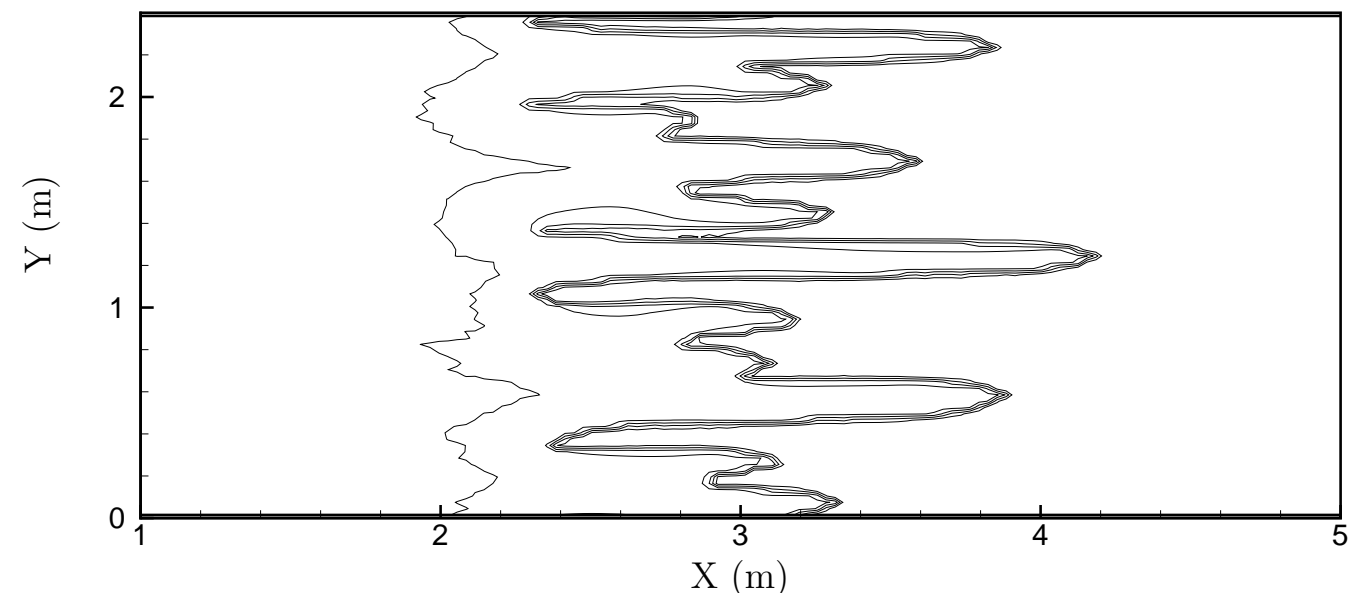

Figure 5.10: Grid Convergence (a) 15,480 (b) 61,920 (c) 141,120 Grid Blocks. Lines represent gas saturation contour levels with 0.02 increments, from $S_{g}=0.02$ (right) to $S_{g}=0.10$ (left). 

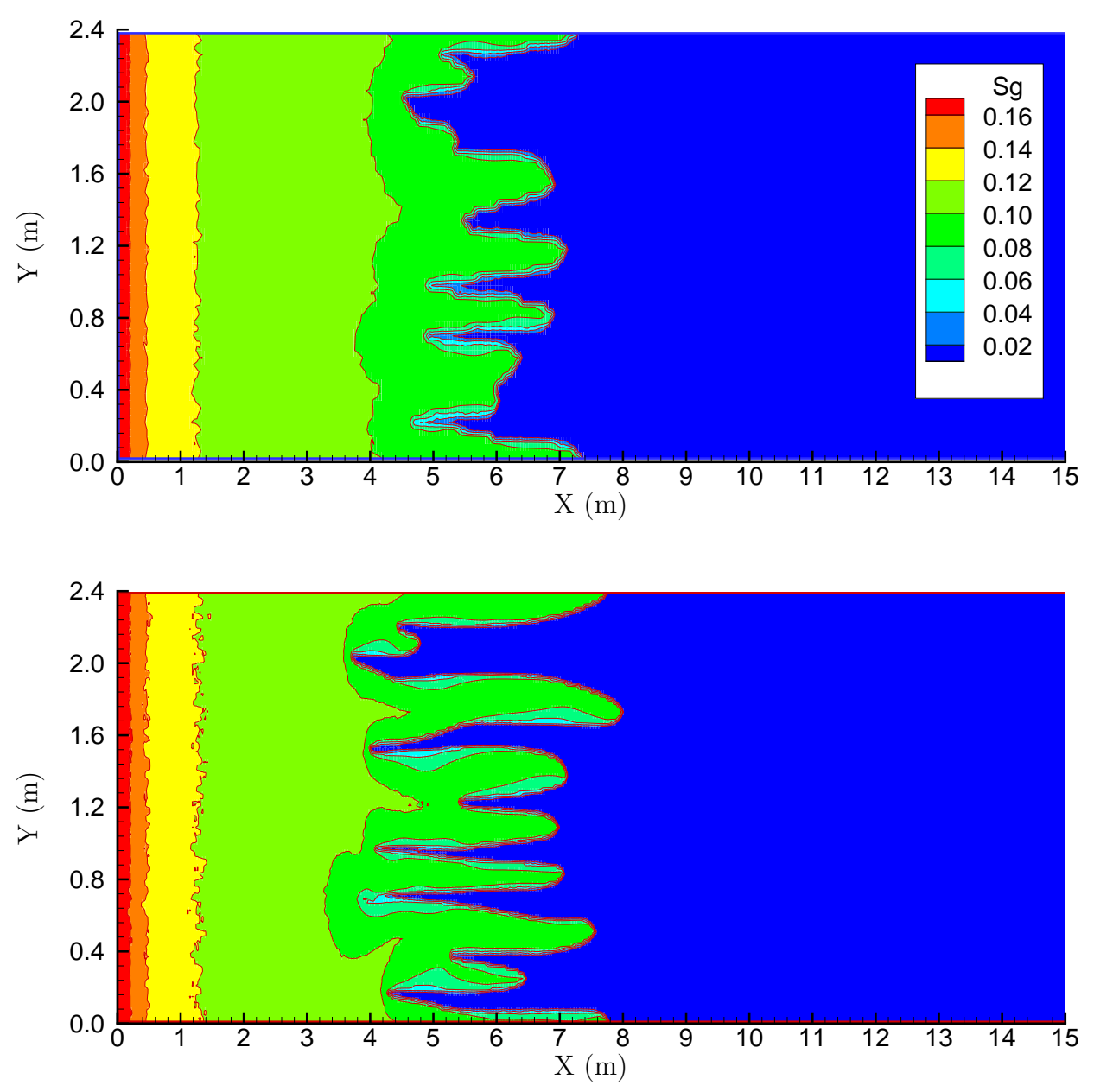

Figure 5.11: Coarse grid (30,000 blocks, scalar machine, top) and fine grid (203,200 blocks, massively parallel scheme, bottom) simulations of viscous fingering. 


\subsubsection{Boundary effects}

As mentioned before, the onset of instabilities is influenced by truncation and round-off errors. This problem becomes particularly relevant for no-flow boundary conditions. Figure 5.12a shows that fingers tend to grow more rapidly along such a boundary, creating an artificial, not realistic flow behavior. In order to avoid preferential flow along these boundaries they are "glued" together and linked by providing additional connections. The result is a two dimensional grid that is wrapped around the mantle of a cylinder, without no-flow boundaries (Figure 5.13).

\subsubsection{Computer Performance}

The simulations show that the overall computer performance depends primarily on the following aspects: (1) problem size (domain and gridblock size vs. injection rates); (2) mobility ratio; (3) the magnitude of the capillary effects. Single processor simulations proceed in a matched fashion on the three platforms. Finger evolution was tracked by obtaining printouts at specified times using the TOUGH2's keyword TIMES. This option is not available in the parallel version because the size of the problem implies very big output files. The progression of fingers at different times in the multiple processor version was captured by restarting the TOUGH2-MP run providing the file SAVE generated in the previous run as file INCON. Results obtained from the single (Platform B) and multiple processor versions (Platform C) are almost identical. Minor differences in convergence behavior, time step size and number of iterations, are due to the use of different solvers. A $308 \times 120$ grid was used to compare the numerical

results obtained with the two versions. In all the cases a similar finger structure is preserved. When using 16 processors, CPU times in the parallel implementation are reduced by a factor of 40 . Because this problem is of modest size, the addition of more processors provides only a marginal gain in speed up. For larger problems though, 
the parallel-code simulations show a better than linear speedup until they reach a saturation point. Speedup here is defined to be relative to the performance with 16 processors. For additional information on the computer performance of the TOUGH2 parallel version the reader is referred to $\mathrm{Wu}$ et al. [2002].

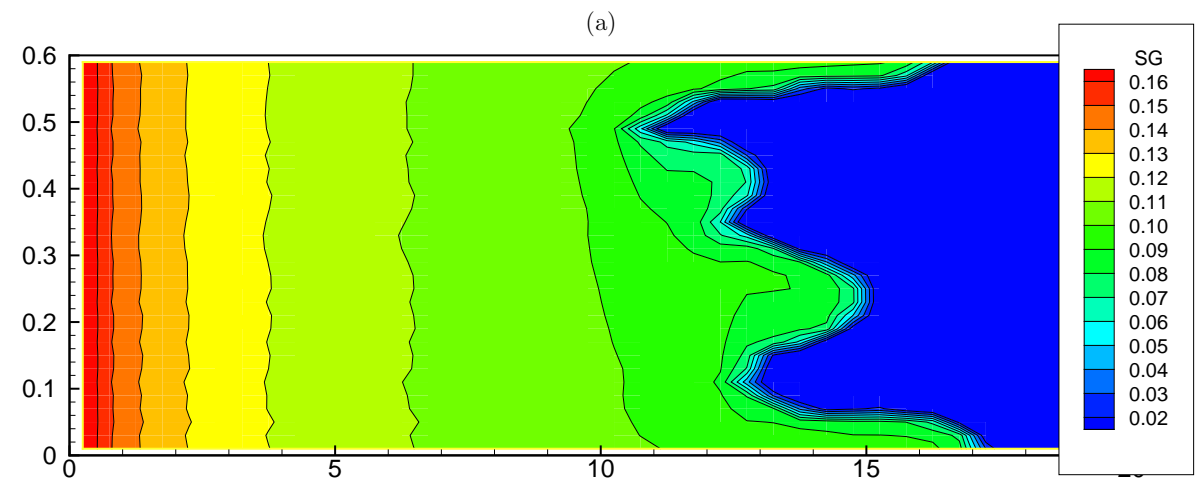

(b)

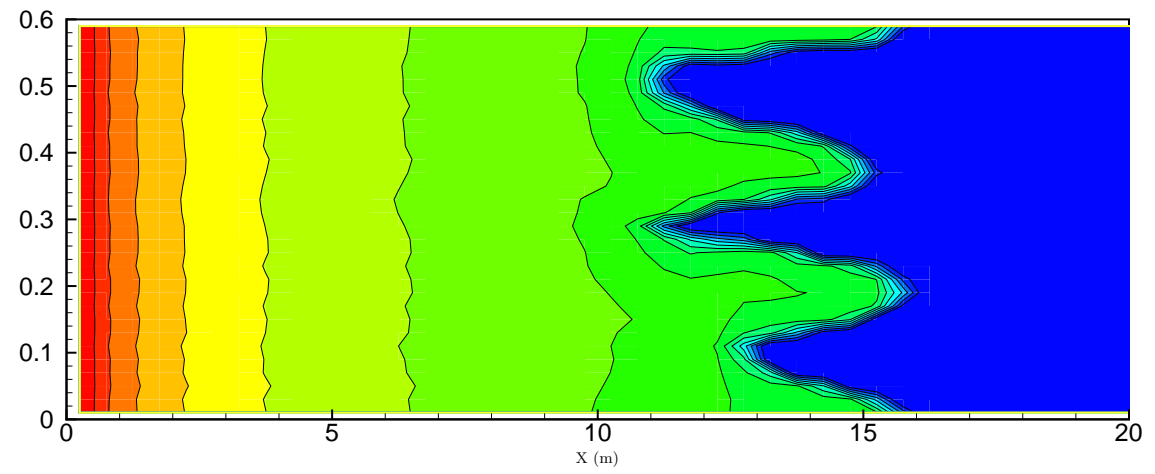

Figure 5.12: No-flow boundary conditions effect. (a) top and bottom no-flow boundaries. (b) top and bottom boundaries connected.

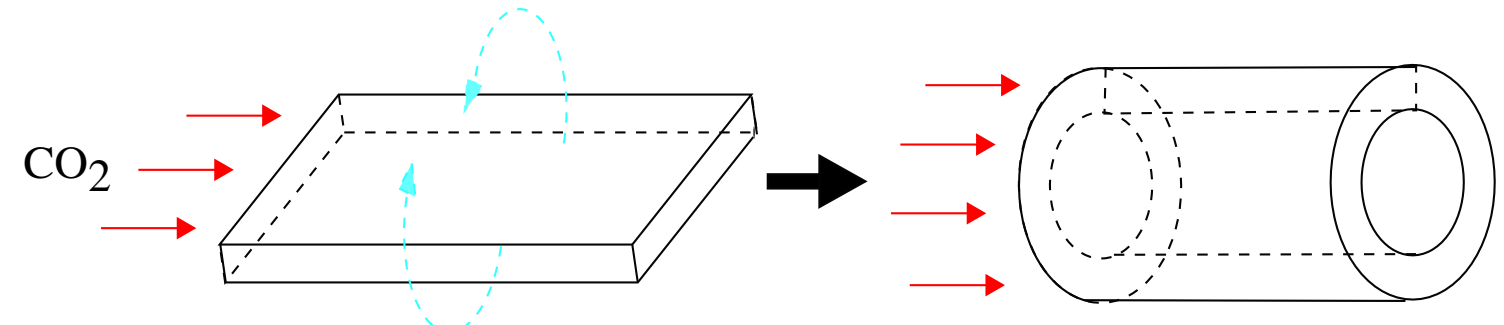

Figure 5.13: No flow boundary conditions (left) can be eliminated by creating artificial 2-D horizontal cylinder type grids (right). 


\subsection{Results and applications}

Chuoke et al. [1959] were the first to provide a theoretical analysis of the onset of fingering, using linear stability analysis. They assume that capillarity acts on a single macroscopic interface, in other words, that there is complete displacement of one fluid by other. An effective interfacial tension, $\sigma^{*}$ was introduced to account for the pressure change across the interface.

\subsubsection{Finger spacing}

For the purpose of the analysis presented in this section we will consider the horizontal displacement of a gas-liquid interface as described by Chuoke et al. [1959]. The region of the invading gas is labelled " 1 ", the region of the invaded liquid " 2 ". Because of the lower gas viscosity the displacement will be unstable. The frontal instabilities will continue to grow at a rate that is dependent on the wavelength of the perturbation. The expected finger spacing is given by the wave length of maximum growth rate,

$$
\gamma_{m}=\sqrt{3} \gamma_{c}
$$

where $\gamma_{c}$ is the wave number of marginal stability, given for horizontal flow by

$$
\gamma_{c}=2 \pi\left[\frac{\sigma^{*}}{\left(\frac{\mu_{2}}{k_{2}}-\frac{\mu_{1}}{k_{1}}\right) v}\right]^{1 / 2}
$$

For the flow system under consideration (Figure 5.3) we have $k_{1}=k_{2}=k$, so that Equation 5.17 can be written as

$$
\gamma_{m}^{2}=\frac{12 \pi^{2} k}{C a}
$$


Here, $C a$ is the local capillary number given for large viscosity ratio $\left(\mu_{2} \gg \mu_{1}\right)$ by

$$
C a=\frac{\mu_{2} v}{\sigma^{*}}
$$

The effective interfacial tension, $\sigma^{*}$, provides a link between the pore and the porous media continuum level. It is important to remind the reader that the previous analysis is based on the assumption that two macroscopic flow regions exist and furthermore that they are separated by an abrupt macroscopic interface. $\sigma^{*}$ allows the pressure in the two regions across the interface to be related to the curvature of the interface. The effective interfacial tension is generally assumed to be directly proportional to the true interfacial tension $\sigma$.

In order to explore the validity of Chuoke's approximation a series of numerical simulation was performed with two hypothetical fluids, $\mu_{l} / \mu_{g}=5500$, so that a high mobility shock can be obtained and maintained due to capillary effects. Again, the relative permeability functional forms given by equations 5.12 and 5.14 are used. Figure 5.14 shows the expected finger width using Equation 5.19 for an intrinsic permeability of $k=10^{-10} \mathrm{~m}^{2}$. The symbols represent the average finger width obtained from numerical simulations, showing good agreement between Equation 5.19 and simulated results. More analysis is required though to assess the validity of Chuoke's model on a more general continuum model. 


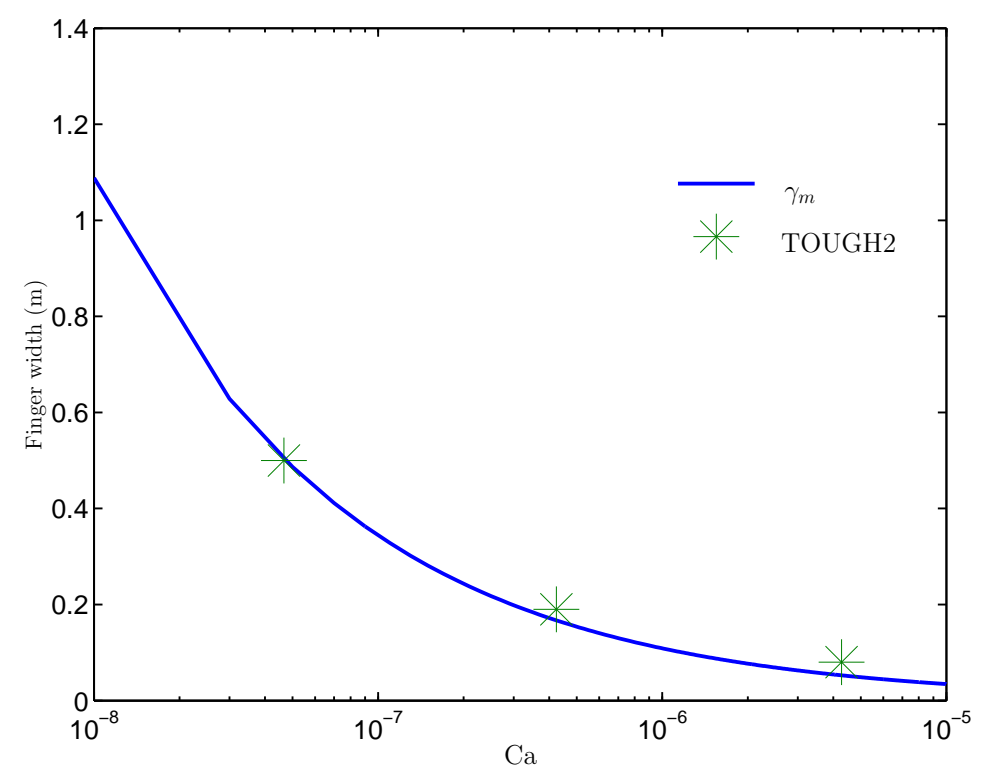

Figure 5.14: Finger Wave-Length.

\subsubsection{Finger growth}

Figure 5.15 shows gas saturations at three different times in a two-dimensional displacement obtained on a $616 \times 240$ grid. Viscous fingering is triggered by variations in the permeability with a maximum modification of $5 \%\left(0.95 \times \mathbf{k} \leq \mathbf{k}_{\text {mod }} \leq 1.05 \times \mathbf{k}\right.$, with $\left.\mathbf{k}=10^{-10}\right)$. Figure 5.16a shows the location of the saturation front $\left(X_{f}(t)\right)$ calculated as a uniform displacement according to the Buckley-Leverett solution together with the absolute finger length $(\Omega(t))$ obtained from the simulation results at 11 times. The relative finger length is calculated as:

$$
\omega(t)=\frac{\Omega(t)}{X_{f}(t)}
$$

The relative finger length shown in Figure 5.16b increases at first, and later appears to reach an asymptotic value. 


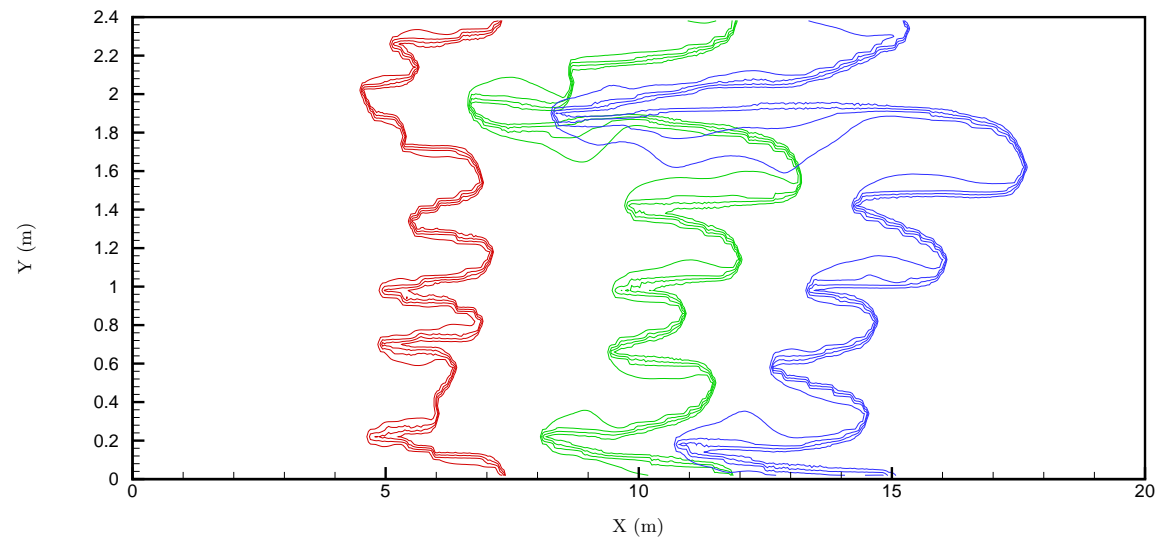

Figure 5.15: Finger Growth. Lines represent frontal gas saturation contour levels $\left(0.08 \geq S_{g} \geq 0.02\right)$ at $T=\left(1.0 \times 10^{7}, 2.0 \times 10^{7}, 3.0 \times 10^{7}\right) \mathrm{sec}$.

(a)

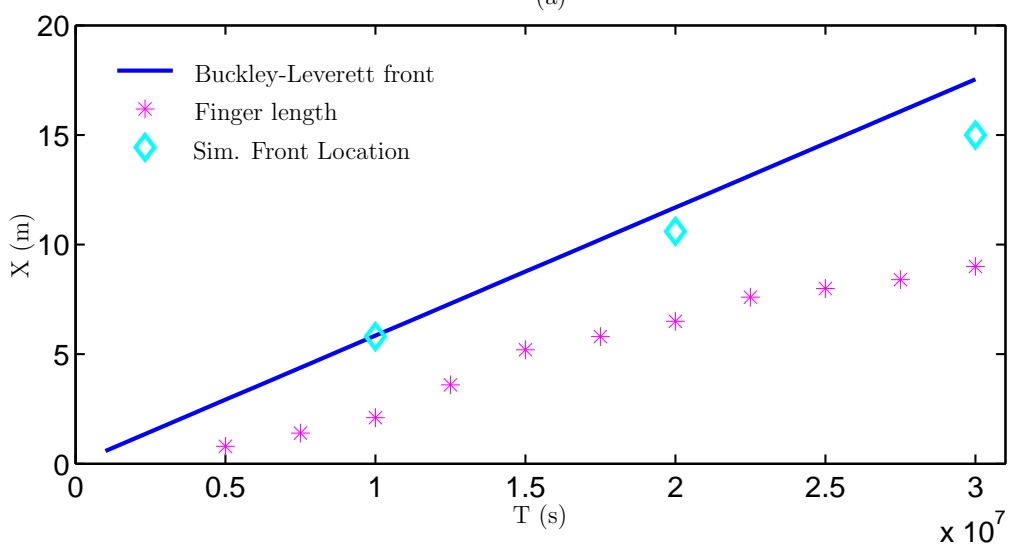

(b)

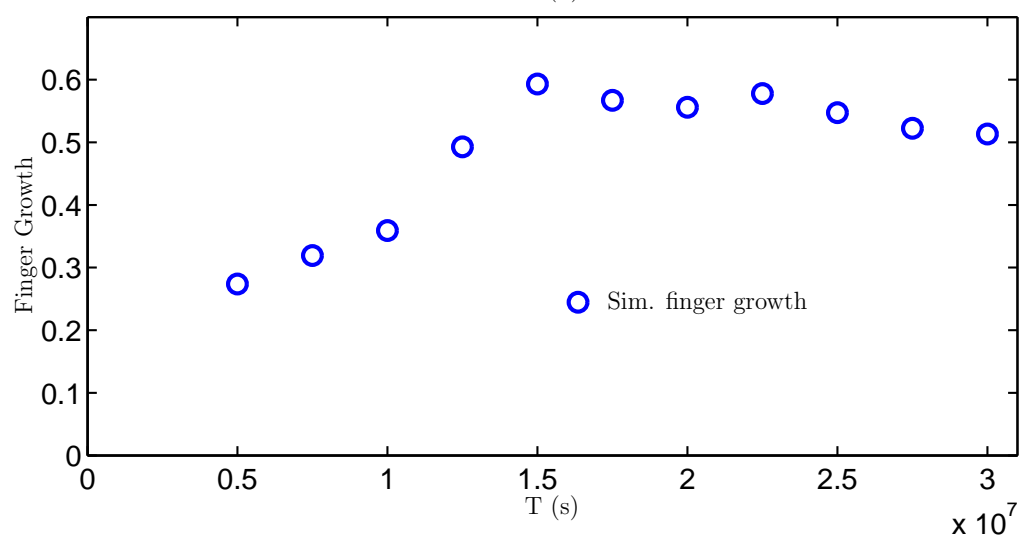

Figure 5.16: High-resolution simulation results for finger growth. 


\subsection{Viscous fingering simulations with ECO2}

Figure 5.17 compares fingering predictions obtained from ECO2 (top frame) with results from EOS3 (bottom frame). Ignoring capillary effects (i.e assuming a BuckleyLeverett solution), the location of the saturation front at time $t$ is given by:

$$
X_{\text {front }}=\frac{q_{t} t}{A \phi}\left(\frac{\partial f_{g}}{\partial S_{g}}\right)_{S_{f}}
$$

where $q_{t}$ is the volumetric gas flow rate, $A$ is the cross-sectional area, and $\left(\frac{\partial f_{g}}{\partial S_{g}}\right)_{S_{f}}$ is the partial derivative of the gas fractional flow function with respect to saturation, evaluated at the frontal saturation. Simulations with ECO2 and EOS3 use the same grid and injection parameters. Because the injection was given in terms of mass rates the actual front location in the simulations has to be scaled by the ratio of the densities $\left(\frac{\rho_{c o 2}}{\rho_{a i r}}\right)$. The results show that fingers are more defined in simulations with EOS3 than with ECO2. The saturation front is more spread when simulations are made with ECO2. 

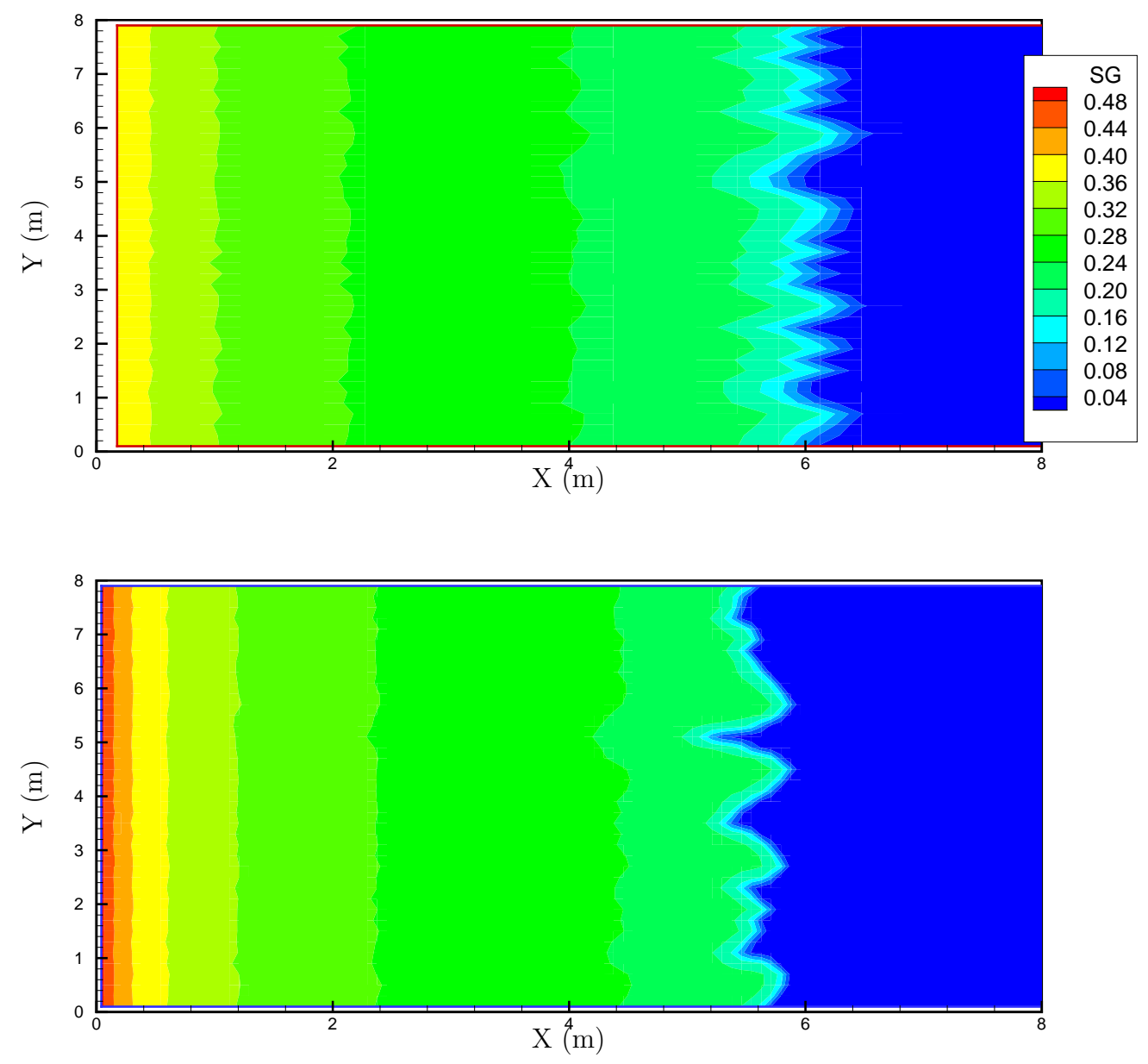

Figure 5.17: Viscous fingering predictions obtained from ECO2 (top) and EOS3 (bottom). 



\section{Chapter 6}

\section{Conclusions}

An initial exploration of the fluid dynamics of carbon dioxide disposal into saline aquifers is presented in this dissertation. Newly developed simulation capabilities are used to analyze the overall multiphase flow behavior under a variety of simplified geohydrologic conditions. The work was motivated by the need for engineering tools for evaluating the feasibility of geologic disposal of $\mathrm{CO}_{2}$, screening favorable and unfavorable conditions, and designing and operating future disposal systems.

In the first part of this work, a detailed study of thermodynamics and thermophysical properties of water, carbon dioxide and of mixtures of $\mathrm{CO}_{2}$ with saline waters was conducted. From this study, a set of simple, yet accurate correlations for fluid property description were proposed (Chapter 2). Then, those numerical representations of thermodynamic properties were incorporated into the new equation of state module for TOUGH2 presented in Chapter 3.

The model was validated using the basic problem of $\mathrm{CO}_{2}$ injection into a radially symmetric brine aquifer (Chapter 3). This problem was also used to study a variety of processes including: (1) Two-phase flow of $\mathrm{CO}_{2}$ and $\mathrm{H}_{2} \mathrm{O}$ subject to relative permeability and capillary effects, (2) change of fluid density, viscosity, and $\mathrm{CO}_{2}$ solubility with pressure and salinity, (3) formation dry-out with precipitation of salt, 
and (4) permeability reduction due to salt precipitation. A prototypical problem for $\mathrm{CO}_{2}$ injection into a layered heterogeneous formation from a horizontal well was analyzed using a simplified 2-D vertical section of the Sleipner Vest field storage system. Simulation results show that the transport in this kind of system is dominated by buoyancy effects and that low permeability layers control vertical migration of $\mathrm{CO}_{2}$. In addition, the model was applied to study the role of enhanced $\mathrm{CO}_{2}$ dissolution by convective mixing (Section 4.4).

Chapter 5 described flow simulations exploring hydrodynamic instability during immiscible displacement in porous media. Simulation results show that TOUGH2 is capable of capturing viscous fingering when there is a displacement of a viscous fluid by a less viscous one. Furthermore, the preliminary results obtained show agreement with classic instability analysis of immiscible displacements in porous media [Chuoke et al., 1959]. Capillary effects act to stabilize the displacement at higher wavelengths. Thus, for higher capillary numbers smaller finger widths are expected.

The analysis was based on two-dimensional (2D) high-resolution numerical simulations that imply the absence of gravity override; hysteresis in relative permeability and capillary pressure was not considered. Some of the simulations assumed an air-like gas with $\mathrm{CO}_{2}$ viscosity rather than $\mathrm{CO}_{2}$ itself. This is of course a rough simplification of the real $\mathrm{CO}_{2}-\mathrm{H}_{2} \mathrm{O}$ system and does not accurately represent certain processes. For example, $\mathrm{CO}_{2}$ dissolution into the water phase that counters the tendency for fingering is underestimated, as was shown in Section 5.6. Nevertheless, the onset of instabilities, finger width and growth can appropriately be studied using this approach.

The simulations examined the injection of $\mathrm{CO}_{2}$ into a domain originally saturated with water. For aquifer disposal conditions, the use of common relative permeability functions gives a mobility ratio less than unity $\left(\lambda_{\text {shock }}<1\right)$, resulting in a stable displacement. When quadratic and cubic relative permeability functions are used, or 
when extreme shape factors are applied to other conventional relative permeability functions such as the van Genuchten [1980], an unstable displacement may occur with moderate mobility ratios of up to 2.5. In addition, both capillary effects and dissolution of $\mathrm{CO}_{2}$ in the aqueous phase will counter the tendency for fingering. Tchelepi and Orr [1994] found that, for miscible displacement in reservoirs, the distribution of permeability rather than hydrodynamic instability dominates fluid displacement. This will likely also be the case for $\mathrm{CO}_{2}$ injection into saline aquifers. Low-viscosity $\mathrm{CO}_{2}$ will find preferential flow paths easily and the heterogeneity structure of the aquifer will determine the extent of channelling and by-passing.

Several issues considered in this investigation deserve further investigation and should be subject of future research as follows:

1. Enhancements of phase equilibria models. Integral consideration of the complete $\mathrm{CO}_{2}-\mathrm{H}_{2} \mathrm{O}-\mathrm{NaCl}$ system.

2. Thermophysical properties of gas mixtures. Flue gases originating from combustion of fossils fuels will in addition to $\mathrm{CO}_{2}$, oxygen and water vapor contain other gases such as $\mathrm{N}_{2}, \mathrm{NO}_{x}, \mathrm{SO}_{x}$ and noble gases.

3. Field tests. Our research was focused on the physics of flow, site-specific data from aquifers will be required to explore numerous important issues concerning $\mathrm{CO}_{2}$ sequestration. Thus, case studies will be an important element of future research.

4. Higher resolution simulations. Definite answers to the viscous fingering problem using the module ECO2 will require high resolution 3-D grids at a high computational cost. Explore the possibility of upscaling or ways to compute average behavior in coarser grids.

5. 3-D simulations in heterogeneous systems. 
6. Fully coupled hydro-mechanical3-D simulations in heterogeneous systems.

7. Laboratory scale experiments.

In this dissertation a better understanding of the fundamental flow processes and mechanisms that are related to $\mathrm{CO}_{2}$ disposal into brine formations was provided through the application of the numerical simulator TOUGH2 to simple, suitable and relevant test problems. A complete evaluation of the feasibility of geologic carbon disposal in aquifers will require the study of several other issues that are beyond the scope of this work including chemical and hydro-mechanical coupled processes. In addition, it is not yet possible to predict with confidence storage volumes and integrity over long time periods of the target formations, and many additional issues must be addressed to reduce costs, gain public acceptance and ensure safety. 


\section{Nomenclature}

What follows is a partial list of symbols used in the dissertation. The same symbol may be used in different contexts to denote different variables. The equation and page numbers refer to the first time the variable is introduced in the text.

\section{Greek symbols}

$\delta \quad$ Increment, Eq. (2.8), p. 22.

$\gamma_{c} \quad$ Wave length of marginal stability $[m]$, Eq. (5.18), p. 112.

$\gamma_{m} \quad$ Wave length of maximum growth rate $[m]$, Eq. (5.17), p. 112.

$\lambda_{c} \quad$ Critical wave length $[m]$, Eq. (4.5), p. 88.

$\lambda_{T} \quad$ Total mobility $[(m \cdot s) / k g]$, Eq. (5.10), p. 98 .

$\lambda_{\alpha} \quad$ Mobility of the $\alpha$-phase $[(m \cdot s) / k g]$, Eq. (5.10), p. 98.

$\mu_{i}^{\alpha} \quad$ Chemical potential of $i$ th component in phase $\alpha[J / m o l]$, Eq. (2.40), p. 36 .

$\mu_{\beta} \quad$ viscosity of the $\beta$-phase $[\mathrm{kg} /(\mathrm{m} \cdot \mathrm{s})]$, Eq. (3.5), p. 52.

$\omega \quad$ Pitzer's acentric factor, p. 17.

$\phi \quad$ Fugacity coefficient, Eq. (2.46), p. 37.

$\phi \quad$ Porosity, Eq. (3.2), p. 51.

$\phi_{f} \quad$ Active flow porosity, Eq. (3.31), p. 64.

$\rho \quad$ Mass density $\left[\mathrm{kg} / \mathrm{m}^{3}\right]$, p. 14 .

$\rho_{\beta} \quad$ Density of the $\beta$-phase $\left[\mathrm{kg} / \mathrm{m}^{3}\right]$, Eq. (3.2), p. 51 .

$\sigma^{*} \quad$ Effective interfacial tension $[N / m]$, Eq. (5.20), p. 113.

$\tau_{\beta} \quad \beta$-phase tortuosity, Eq. (3.9), p. 53. 


\section{Roman symbols}

$\bar{V} \quad$ Partial molar volume $\left[\mathrm{m}^{3} / \mathrm{mol}\right]$, Eq. (2.48), p. 39.

g Vector of gravitational acceleration $\left[\mathrm{m} / \mathrm{s}^{2}\right]$, Eq. (3.5), p. 52.

k Intrinsic permeability tensor $\left[m^{2}\right]$, Eq. (3.5), p. 52 .

$\overline{\mathbf{D}}_{\beta}^{(\kappa)} \quad$ Hydrodynamic dispersion tensor $\left[\mathrm{m}^{2} / \mathrm{s}\right]$, Eq. (3.6), p. 53.

A Molar Helmholtz Energy $[\mathrm{J} / \mathrm{mol}]$, p. 19.

a Coefficient of the van der Waals equation of state [bar, Pa], Eq. (2.5), p. 17.

$A^{*} \quad$ Coefficient of cubic equations of state, Eq. (2.6), p. 17.

$b \quad$ Coefficient of the van der Waals equation of state $\left[\mathrm{m}^{3} / \mathrm{mol}\right]$, Eq. (2.5), p. 17 .

$B^{*} \quad$ Coefficient of cubic equations of state, Eq. (2.6), p. 17.

$C^{*} \quad$ Coefficient of cubic equations of state, Eq. (2.6), p. 17.

$C_{p} \quad$ Heat capacity $[J /(\mathrm{mol} \cdot K]$, Eq. $(2.18)$, p. 24 .

$C_{R} \quad$ Specific heat of the rock grains $[\mathrm{J} /(\mathrm{kg} \cdot \mathrm{K})]$, Eq. (3.3), p. 52.

Ca Local capillary number, Eq. (5.19), p. 113.

$d \quad$ Differential, Eq. (2.8), p. 22.

$d_{\beta}^{(\kappa)} \quad$ Molecular diffusion for component $\kappa$ in the $\beta$-phase $\left[m^{2} / s\right]$, Eq. (3.9), p. 53 .

$F_{g} \quad$ Gas fractional flow, Eq. (5.9), p. 97.

$f_{i}^{\alpha} \quad$ Fugacity of $i$ th component in phase $\alpha[b a r, P a]$, Eq. (2.45), p. 37.

G Molar Gibbs energy [J/mol], Eq. (2.14), p. 23.

$H \quad$ Molar Enthalpy $[\mathrm{J} / \mathrm{mol}]$, p. 21.

$h \quad$ Enthalpy $[\mathrm{J} / \mathrm{kg}]$, p. 21.

$H_{\text {sol }} \quad$ Heat of dissolution $[\mathrm{J} / \mathrm{kg}]$, Eq. (2.56), p. 43.

$K_{H_{i}} \quad$ Henry's coefficient [bar, Pa], Eq. (2.36), p. 35.

$k_{r \beta} \quad$ Relative permeability of the $\beta$-phase, Eq. (3.5), p. 52.

$M^{(\kappa)} \quad$ Mass of component $(\kappa)[k g]$, Eq. (3.2), p. 51.

$n \quad$ Number of chemical species, Eq. (2.1), p. 12.

$n \quad$ Number of moles, Eq. (2.8), p. 22. 
NPH Number of phases, Eq. (3.24), p. 59.

P Thermodynamic pressure [bar, Pa], p. 14 .

$\mathrm{P}_{\mathrm{CO}_{2}} \quad \mathrm{CO}_{2}$ partial pressure [bar, $\left.\mathrm{Pa}\right]$, Eq. (3.30), p. 63.

$Q \quad$ Heat $[J]$, Eq. (2.8), p. 22 .

$R \quad$ The universal gas constant $\left[\mathrm{bar} \cdot \mathrm{cm}^{3} /(\mathrm{mol} \cdot K)\right]$, Eq. (2.3), p. 16 .

$r \quad$ Number of phases, Eq. (2.1), p. 12.

$S \quad$ Molar entropy $[J /(K \cdot m o l)]$, Eq. (2.10), p. 22.

$S_{\beta} \quad$ Saturation of the $\beta$-phase, Eq. (3.2), p. 51 .

$S_{g r} \quad$ Irreducible gas saturation, p. 70.

$S_{l r} \quad$ Irreducible water saturation, p. 70.

T Temperature $\left[{ }^{\circ} \mathrm{C}, K\right]$, p. 14 .

$U \quad$ Molar internal energy $[\mathrm{J} / \mathrm{mol}]$, Eq. (2.8), p. 22.

$u_{\beta} \quad$ Specific internal energy of the $\beta$-phase $[\mathrm{J} / \mathrm{kg}]$, Eq. (3.3), p. 52 .

$V \quad$ Molar volume $\left[\mathrm{m}^{3} / \mathrm{mol}\right]$, Eq. (2.5), p. 17.

$W \quad$ Work $[J]$, Eq. (2.8), p. 22.

$X_{i} \quad$ Mass fraction of $i$ th component in the liquid phase, Eq. (2.56), p. 43.

$x_{i} \quad$ Mole fraction of the $i$ th component in the liquid phase, Eq. (2.35), p. 35.

$Y_{i} \quad$ Mass fraction of the $i$ th component in the gas phase, Eq. (2.54), p. 42.

$y_{i} \quad$ Mole fraction of the $i$ th component in the gas phase, Eq. (2.35), p. 35 .

Z Gas compressibility factor, Eq. (2.4), p. 16.

\section{Subscripts}

b Brine, Eq. (2.55), p. 43.

c $\quad$ Critical property, p. 17.

$g \quad$ Gas phase, Eq. (2.53), p. 41.

$l \quad$ Liquid phase, p. 14.

$p \quad$ Iteration level, Eq. (3.27), p. 60.

$r \quad$ Reduced property, Eq. (2.4), p. 16. 
$v \quad$ Vapor phase, p. 14.

\section{Superscripts}

0 Thermophysical property of pure $\mathrm{CO}_{2}$ as an ideal gas, Eq. (2.24), p. 24 .

$\infty \quad$ Thermophysical property at infinite dilution, Eq. (2.51), p. 39.

$k \quad$ Time step level, p. 60 . 


\section{Bibliography}

Altunin, V. V. (1975). Thermophysical properties of carbon dioxide. Publishing House of Standards, Moscow (in Russian).

Altunin, V. V. and Gadetskii, O. G. (1971). Equation of state and thermodynamic properties of liquid and gaseous carbon dioxide. Thermal Engineering, 18(3):120125. Teploenergetika, 18, (3), 81-84 (Russian).

Andersen, G., Probst, A., Murray, L., and Buttler, S. (1992). An accurate PVT model for geothermal fluids as represented by $\mathrm{H}_{2} \mathrm{O}-\mathrm{CO}_{2}-\mathrm{NaCl}$ mixtures. In Jr., $\mathrm{H}$. J. R., Horne, R. N., Kruger, P., Miller, F. G., Brigham, W. E., and Cook, J. W., editors, Proceedings, Seventeenth Workshop on Geothermal Reservoir Engineering, pages 239-248, Stanford, California. Standford University.

Angus, S., Armstrong, B., and de Reuck, K. M. (1976). International Thermodynamics Tables of the Fluid State Carbon Dioxide. International Union of Pure and Applied Chemistry, Pergamon Press, Oxford.

Bachu, S. (2000). Sequestration of $\mathrm{CO}_{2}$ in geological media: criteria and approach for site selection in response to climate change. Energy Conservation and Management, 41:953-970.

Battistelli, A., Calore, C., and Pruess, K. (1997). The simulator TOUGH2/EWASG 
for modelling geothermal reservoirs with brines and non-condensible gas. Geothermics, 26(4):437-464.

Bear, J. (1972). Dynamics of Fluids in Porous Media. Dover Publications, Inc., New York, NY.

Binning, P. and Celia, M. A. (1999). Practical implementation of the fractional flow approach to multi-phase flow simulation. Adv. Water Resour., 22(5):461-478.

Blunt, M., Fayers, F. J., and Orr, F. M. (1993). Carbon dioxide in enhanced oil recovery. Energy Conservation and Management, 334:1197-1204.

Blunt, M. J., Barker, J. W., Rubin, B., Mansfield, M., Culverwell, I. D., and Christie, M. A. (1992). A predictive theory for viscous fingering in compositional displacement. In SPE/DOE Eigth Symposium on Enhanced Oil Recovery, Tulsa, Oklahoma. SPE 24129.

Bowers, T. S. (1982). Calculation of the Thermodynamic and Geochemical Consequences of Nonideal Mixing in the System $\mathrm{H}_{2} \mathrm{O}-\mathrm{CO}_{2}-\mathrm{NaCl}$ on Phase Relations in Geologic Systems. PhD thesis, University of California at Berkeley.

Bowers, T. S. and Helgeson, H. C. (1983). Calculation of the thermodynamic and geochemical consequences of nonideal mixing in the system $\mathrm{H}_{2} \mathrm{O}-\mathrm{CO}_{2}-\mathrm{NaCl}$ on phase relations in geologic systems: Equation of state for $\mathrm{H}_{2} \mathrm{O}-\mathrm{CO}_{2}-\mathrm{NaCl}$ fluids at high pressures and temperatures. Geochimica et Cosmochimica Acta, 47:1247-1275.

Buckley, S. E. and Leverett, M. C. (1942). Mechanism of fluid displacement in sands. Petrol. Trans. AIME, 146:107.

Camhi, E., Meiburg, E., and Ruith, M. (2000). Miscible rectilinear displacements with gravity override. Part 2. Heterogeneous porous media. J. Fluid Mech., 420:259-276. 
Carroll, J. J., Slupsky, J. D., and Mather, A. E. (1991). The solubility of carbon dioxide in water at low pressure. JPHCH, 20(6).

Chou, I. M. (1987). Phase relations in the system NaCl-KCl-H $\mathrm{N}_{2} \mathrm{O}$. III: solubilities of halite in vapor-saturated liquida above $445{ }^{\circ} \mathrm{c}$ and redetermination of phase equilibrium properties in the system $\mathrm{NaCl}_{-} \mathrm{H}_{2} \mathrm{O}$. Geochimica et Cosmochimica Acta, 51:1965-1975.

Christie, M. A. (1989). High-resolution simulations of unstable flows in porous media. SPE Reserv. Eng., pages 297-303. SPE 16005.

Chuoke, R. L., van Meurs, P., and van der Poel, C. (1959). The instability of slow, immiscible, viscous liquid-liquid displacements in permeable media. Petrol. Trans. AIME, 216:188-194.

Corey, A. T. (1954). The interrelation between gas and oil relative permeabilities. Producers Monthly, pages 38-41.

Cramer, S. D. (1982). The solubility of methane carbon dioxide, and oxygen in brines from $0^{\circ}$ to $300^{\circ}$ c. Report of Investigations 8706, US Bureau of Mines, U.S.A.

Crone, S., Bergins, C., and Strauss, K. (2002). Multiphase flow in homogeneous porous media with phase change. part i: Numerical modeling. (49):291-312.

de Marsily, G. (1986). Quantitative Hydrogeology: Groundwater Hydrology for Engineers. Academic Press, San Diego, CA.

De Wit, A. and Homsy, G. M. (1999a). Nonlinear interactions of chemical reactions and viscous fingering in porous media. 11(5):949-951.

De Wit, A. and Homsy, G. M. (1999b). Viscous fingering in reaction-diffusion systems. J. Chem. Phys., 110(17):8663-8675. 
DOE (1999). Carbon sequestration research and development. Technical Report DOE/SC/FE-1, U.S. Department of Energy, Washington, DC, USA.

DOE (2003). Doe, penn state to establish gas storage technology consortium. Technical Report DOE-Techline: September 11, 2003, U.S. Department of Energy.

Doughty, C. and Pruess, K. (1992). A similarity solution for two-phase water, air, and heat flow near a linear heat source in a porous medium. J. of Geophysical Research, 97(B2):1821-1838.

Doughty, C. and Pruess, K. (2003). Modeling supercritical $\mathrm{CO}_{2}$ injection in heterogeneous porous media. In Proceedings, TOUGH Symposium, Berkeley, California.

Drummond, S. E. (1981). Boiling and Mixing of Hydrothermal Fluids: Chemical effects on Mineral Precipitation. PhD Dissertation, Pennsylvania State University.

Duan, Z., Møller, N., and Weare, J. H. (1995). Equation of state for the $\mathrm{NaCl}_{-} \mathrm{H}_{2} \mathrm{O}-$ $\mathrm{CO}_{2}$ system: Prediction of phase equilibria and volumetric properties. Geochimica et Cosmochimica Acta, 59(14):2869-2882.

Duan, Z. and Sun, R. (2003). An improved model calculating $\mathrm{CO}_{2}$ solubility in pure water and aqueous $\mathrm{NaCl}$ solutions from 273 to $533 \mathrm{k}$ and from 0 to 2000 bar. Chemical Geology, (193):257-271.

Ennis-King, J. and Paterson, L. (2003). Role of convective mixing in yhe long-term storage of carbon dioxide in deep saline formations. In SPE Annual Technical Conference and Exhibition, Denver, Colorado. SPE 84344.

Evelein, K. A., Moore, R. G., and Heidemann, R. A. (1976). Correlation of the phase behavior in the systems hydrogen sulfide-water and carbon dioxide-water. Ind. Eng. Chem. Proc. Des. Dev., (3):423-428. 
Fenghour, A., Wakeman, W. A., and Vesovic, V. (1998). The viscosity of carbon dioxide. J. Phys. Chem. Ref. Data, 27(1):31-44.

García, J. E. (2001). Density of aqueous solutions of $\mathrm{CO}_{2}$. Technical Report LBNL49023, Lawrence Berkeley National Laboratory, Berkeley, CA.

Gibbs, J. W. (1876). On the equilibrium of heterogeneous substances, Part I. Transactions of the Connecticut Academy, III:108-248.

Gibbs, J. W. (1878). On the equilibrium of heterogeneous substances, Part II. Transactions of the Connecticut Academy, III:343-524.

GSSSD (1987). Carbon dioxide. coefficients of dynamic viscosity, thermal conductivity at temperatures $220-1000 \mathrm{~K}$ at pressures ranging from dilute gas state to $100 \mathrm{MPa}$. Tables of Standard Reference Data, Standard Press 101-87, Bureau of Standard and Reference Data of USSR, Moscow.

Gudmundsson, J.-S. and Thrainsson, H. (1989). Power potential of two-phase geothermal wells. Geothermics, (18):357-366.

Hass, J. L. J. (1976). Physical properties of the coexisting phases and thermochemical properties of $\mathrm{H}_{2} \mathrm{O}$ component in boiling $\mathrm{NaCl}$ solutions. Bulletin 1421-A, USGS, Washington, D.C., USA.

Herzog, H., Drake, E., and Adams, E. (1997). CO 2 capture, reuse, and storage technologies for mitigating global climate change. Technical report, Energy Laboratory, Massachusetts Institute of Technology, Cambridge, MA, USA.

Hill, S. (1952). Channelling in packed columns. Chem. Eng. Sci., I(6):247-253.

Hitchon, B., editor (1996). Aquifer disposal of carbon dioxide. Geoscience Publishing, Sherwood Park, Alberta, Canada. 
Homsy, G. M. (1987). Viscous fingering in porous media. Ann. Rev. Fluid Mech., 19:271-311.

IEA (2001). Putting carbon back into the ground. Technical report, International Energy Agency (IEA) Greenhouse R\&D Programme.

IFC (1967). A Formulation of the thermodynamic properties of ordinary water substance. International Formulation Committee Secretariat, Düsseldorf, Germany.

Juanes, R. (2003). Displacement theory and multiscale numerical modeling of threephase flow in porous media. PhD thesis, University of California at Berkeley.

Katz, D. L. and Lee, R. L. (1990). Natural Gas Engineering: Production and Storage. McGraw-Hill, New York.

Kessel'man, P. M. and Kotloarevskii, P. A. (1965). Thermodynamics properties of carbon dioxide in the temperature range $273-4000 \mathrm{k}$ and at pressures up to 600 bar. Technical report, Lomonosov Odessa Technological Institute.

Korbøl, R. and Kaddour, A. (1995). Sleipner Vest $\mathrm{CO}_{2}$ disposal-injection of removed $\mathrm{CO}_{2}$ into the Utsira formation. ECOMA, 17(5-6):303-308.

Kumagai, A. and Yokoyama, C. (1999). Viscosities of aqueous NaCl solutions containing $\mathrm{CO}_{2}$ at high pressures. J. Chem. Eng. Data, (44):227-229.

Lindeberg, E., Zweigel, P., Gergmo, P., Ghaderi, A., and Lothe, A. (2000). Prediction of $\mathrm{CO}_{2}$ distribution pattern improved by geologic and reservoir simulation and verified by time lapse seismic. In Proceedings, Fifth International Conference on Greenhouse Gas Control Technologies.

Mäder, U. K. and Berman, R. G. (1991). An equation of state for carbon dioxide to high pressure and temperature. American Mineralogist, 76:1547-1559. 
Michaelides, E. E. (1981). Thermodynamics properties of geothermal fluids. Geothermal Resources Council Transactions, (5):361-364.

Miller, C. T., Christakos, G., Imhoff, P. T., McBride, J. F., and Pedit, J. A. (1998). Multiphase flow and transport modeling in heterogeneous porous media: challenges and approaches. Adv. Water Resour., 21(2):77-120.

Moridis, G. J. and Pruess, K. (1998). T2SOLV: An enhanced package of solvers for the TOUGH2 family of reservoir simulation codes. Geothermics, 27(4):415-444.

Morris, R. W. and Turek, E. A. (1986). Optimal temperature-dependent parameters for the redlich-kwong equation of state. In Chao, K. and Robinson, R. L., editors, Equation of State, American Chemical Society Symp., Ser. 300, pages 389-405, Miami, Florida. ACS.

Narasimhan, T. N. and Witherspoon, P. A. (1976). An integrated finite difference model for analyzing fluid flow in porous media. Water Resour. Res., 12(1):57-64.

Oldenburg, C. M. and Pruess, K. (1995). Dispersive transport dynamics in a strongly coupled groundwater-brine flow system. Water Resour. Res., 31(2):289-302.

Orr, F. M. and Taber, J. J. (1984). The use of $\mathrm{co}_{2}$ in enhanced oil recovery. Science, 224:563-569.

O’Sullivan, M. J. (1981). A similarity method for geothermal well test analysis. Water Resour. Res., 17(2):390-398.

Pankiewitz, C. and Meiburg, E. (1999). Miscible porous media displacement in the quarter five-spot configuration. Part 3. Non-monotonic viscosity profiles. J. Fluid Mech., 388:171-195.

Pape, H., Clauser, C., and Iffland, J. (1999). Permeability prediction based on fractal pore-space geometry. Geophysics, 64(5):1447-1460. 
Peaceman, D. W. (1977). Fundamentals of Numerical Reservoir Simulation, volume 6 of Developments in Petroleum Science. Elsevier, Amsterdam.

Phillips, S. L., Igbene, A., Fair, J. A., Ozbek, H., and Tavana, M. (1981). A technical databook for geothermal energy utilization. Technical Report LBL-12810, Lawrence Berkeley National Laboratory, Berkeley, California, USA.

Pitzer, K. S. (1995). Thermodynamics. McGraw-Hill series in advanced chemistry. McGraw Hill, third edition.

Potter, R. W. and Brown, D. L. (1977). The volumetric properties of aqueous sodium chloride solutions from $0^{\circ}$ to $500^{\circ} \mathrm{c}$ at pressures up to 2000 bars based on a regression of available data in the literature. Bulletin 1421-C, USGS, Washington, D.C., USA.

Prausnitz, J. M., Lichtenthaler, R. N., and de Azevedo, E. G. (1986). Molecular Thermodynamics of Fluid-Phase Equilibria. Prentice-Hall Inc., Englewood Cliffs, N.J., second edition.

Pruess, K. (1987). TOUGH user's guide. Technical Report LBL-20700, Lawrence Berkeley National Laboratory, Berkeley, California, USA.

Pruess, K. (2003). Numerical simulation of leakage from a geologic disposal reservoir for $\mathrm{CO}_{2}$, with transitions between super- and sub-critical conditions. In Proceedings, TOUGH Symposium, Berkeley, California.

Pruess, K. and García, J. E. (2002). Multiphase flow dynamics during $\mathrm{CO}_{2}$ disposal into saline aquifers. Environmental Geology, 42(2-3):282-295.

Pruess, K., García, J. E., Kovscek, T., Oldenburg, C., Rutqvist, J., Steefel, C., and $\mathrm{Xu}, \mathrm{T}$. (2002). Intercomparison of numerical simulations codes for geologic disposal of $\mathrm{CO}_{2}$. Technical Report LBNL-51813, Lawrence Berkeley National Laboratory, Berkeley, CA. 
Pruess, K., Oldenburg, C., and Moridis, G. (1999). TOUGH2 user's guide, version 2.0. Technical Report LBNL-43134, Lawrence Berkeley National Laboratory, Berkeley, California, USA.

Pruess, K., Xu, T., Apps, J., and Garcia, J. (2003). Numerical modeling of aquifer disposal of $\mathrm{CO}_{2}$. Soc. Pet. Eng. J., pages 49-60. SPE 83695.

Rah, K. and Eu, B. C. (2001). Density and temperature dependence of the bulk viscosity of molecular liquids: Carbon dioxide and nitrogen. J. Chem. Phys., 114(23):10436.

Reid, R. C., Prausnitz, J. M., and Poling, B. E. (1987). The Properties of Gases and Liquids. McGraw-Hill, fourth edition.

Ruith, M. and Meiburg, E. (2000). Miscible rectilineas displacements with gravity override. Part 1. Homogeneous porous medium. J. Fluid Mech., 420:225-257.

Rumpf, B., Nicolaisen, H., Öcal, C., and Maurer, G. (1994). Solubility of carbon dioxide in aqueous solutions of sodium chloride: Experimental results and correlation. Journal of Solution Chemistry, 23(3):432-448.

Rutqvist, J. and Tsang, C.-F. (2002). A study of caprock hydromechanical changes associated with $\mathrm{CO}_{2}$-injection into a brine formation. Environmental Geology, 42(23):296-305.

Saffman, P. G. and Taylor, S. G. (1958). The penetration of a fluid into a porous medium or Hele-Shaw cell containing a more viscous liquid. Proc. Roy. Soc. London Series A., 245:296-305.

Sahimi, M. (1993). Flow phenomena in rocks: from continuum models to fractals, percolation, cellular automata, and simulated annealing. Reviews of Modern Physics, 65(4):1393-1537. 
Scharlin, P., editor (1996). Carbon Dioxide in Water and Aqueous Electrolyte Solutions, volume 62 of Solubility Data Series. Oxford University Press, Oxford, UK. IUPAC International Union of Pure and Applied Chemistry.

Schofield, P. (1969). Parametric representation of the equation of state near a critical point. Phys. Rev. Letters, 22:606-608.

Schofield, P., Lister, P., and Ho, J. T. (1969). Correlation between critical coefficients and critical exponents. Phys. Rev. Letters, 23:1098-1102.

Smith, J. M., Ness, H. C. V., and Abbott, M. M. (2001). Introduction to Chemical Engineering Thermodynamics. Mc Graw Hill, sixth edition.

Span, R. and Wagner, W. (1996). A new equation of state for carbon dioxide covering the fluid region from the triple-point temperature to $1100 \mathrm{k}$ at pressures up to 800 mpa. J. Phys. Chem. Ref. Data, 25(6):1509-1596.

Spycher, N. F., Pruess, K., and Ennis-King, J. (2003). $\mathrm{CO}_{2}-\mathrm{H}_{2} \mathrm{O}$ mixtures in the geological sequestration of $\mathrm{CO}_{2}$. i. assessment and calculation of mutual solubilities from 12 to 100 c and up to 600 bar. Geochimica et Cosmochimica Acta, 67.

Takenouchi, S. and Kennedy, G. C. (1964). The binary system $\mathrm{h}_{2} \mathrm{O}-\mathrm{CO}_{2}$ at high temperatures and pressures. American Journal of Science, 262:1055-1074.

Tchelepi, H. A. and Orr, F. M. (1994). Interaction of viscous fingering, permeability heterogeneity, and gravity segregation in three dimensions. SPE Reserv. Eng., pages 266-271. (SPE 25235).

Tödheide, K. and Franck, E. U. (1963). Das zweiphasengebeit und die kritische curve im system kohlendioxid-wasser bis zu drucken von 3500 bar. Zeitchrift für Physikalische Chemie Neu Folge, (37):387-401. 
UNFCCC (2002). Climate change information kit. Technical report, United Nations Framework Convention on Climate Change.

van Genuchten, M. T. (1980). A closed-form equation for predicting the hydraulic conductivity of unsaturated soils. 44:892-898.

van Meurs, P. (1957). The use of transparent three-dimensional models for studying the mechanism of flow processes in oil reservoirs. Petrol. Trans. AIME, 210:295301.

Vargaftik, N. B., Vinogradov, Y. K., and Yargin, V. S. (1996). Handbook of Physical Properties of Liquids and Gases. Begell House, New York, third edition.

Verma, A. and Pruess, K. (1988). Thermohydrological conditions and silica redistribution near high-level nuclear wastes emplaced in saturated geological formations. J. of Geophysical Research, 93(B2):1159-1173.

Vesovic, V., Wakeham, W. A., Olchowy, G. A., Sengers, J. V., and Watson, J. T. R. (1990). The transport properties of carbon dioxide. J. Chem. Eng. Data, 19(3):763808.

Vukalovich, M. P. and Altunin, V. V. (1968). Thermophysical Properties of Carbon Dioxide. Collet's.

Welge, H. J. (1952). A simplified method for computing oil recovery by gas or water drive. Petrol. Trans. AIME, 195:91-98.

Wendland, M., Hasse, H., and Maurer, G. (1999). Experimental pressure-temperature data on three- and four-phase equilibria of fluid, hydrate, and ice phases in the system carbon dioxide-water. J. Chem. Eng. Data, 44:901-906. 
Wiebe, R. and Gaddy, V. L. (1940). The solubility of carbon dioxide in water at various temperatures from 12 to $40^{\circ}$ and at pressures to 500 atmospheres: critical phenomena. J. Am. Chem. Soc., (62):815-817.

Wiebe, R. and Gaddy, V. L. (1941). Vapor phase composition of carbon dioxidewater mixtures at varipis temperatures and at pressures to 700 atmospheres. J. Am. Chem. Soc., (63):475-477.

Wiebe, R., Gaddy, V. L., and Heins, C. (1933). The solubility of nitrogen at 50,75, and $100^{\circ}$, at pressures to 700 atmospheres. J. Am. Chem. Soc., (55):947-953.

Wu, Y.-S., Pruess, K., and Chen, Z. X. (1990). Buckley-leverett in composite porous media. Technical Report LBL-28937, Lawrence Berkeley National Laboratory, Berkeley, California.

Wu, Y.-S., Zhang, K., Ding, C., Pruess, K., Elmroth, E., and Bodvarson, G. (2002). An efficient parallel-computing method for modeling nonisothermal multiphase flow and multicomponent transport in porous and fractured media. Adv. Water Resour., $25: 243-261$.

Xu, T., Aps, J. A., and Pruess, K. (2003). Reactive geochemical transport simulation to study mineral trappnig for $\mathrm{CO}_{2}$ disposal in deep aranaceous formations. J. of Geophysical Research, 108(B2). 2071,doi:10.1029/2002JB001979.

Yortsos, Y. and Huang, A. B. (1986). Linear stability analysis of immiscible displacement Part 1 - Simple basic flow profiles. SPE Reserv. Eng., pages 378-390. (SPE 12692).

Zhang, K. (2003). User's manual for TOUGH2_MP vesion 1.0. Technical report, Lawrence Berkeley National Laboratory. 
Zimmerman, W. B. and Homsy, G. M. (1992). Three-dimensional viscous fingering: A numerical study. Phys. Fluids A, 4(9):1901-1914.

Zweigel, P., Hamborg, M., Arts, R., Lothe, A., Sylta, O., and mmeråras, A. T. (2000). Prediction of migration of $\mathrm{CO}_{2}$ injected into an underground depository: Reservoir geology and migration modelling in the sleipner case (North Sea). In Proceedings, Fifth International Conference on Greenhouse Gas Control Technologies. 